\title{
Equivariant covers for hyperbolic groups
}

\author{
ARTHUR BARTELS \\ WOLFGANG LÜCK \\ HOLGER REICH
}

\begin{abstract}
We prove an equivariant version of the fact that word-hyperbolic groups have finite asymptotic dimension. This is important in connection with our forthcoming proof of the Farrell-Jones conjecture for $K_{*}(R G)$ for every word-hyperbolic group $G$ and every coefficient ring $R$.
\end{abstract}

20F65, 20F67; 37D40, 57M07

\section{Introduction}

The asymptotic dimension of a metric space $X$ was introduced by Gromov in [11, p 29]. It can be defined as the smallest number $N$ such that for every $\alpha>0$ there exists an open cover $\mathcal{U}$ of $X$ with the following properties:

- $\operatorname{dim} \mathcal{U} \leq N$

- The Lebesgue number of $\mathcal{U}$ is at least $\alpha$, ie, for every $x \in X$ there is $U \in \mathcal{U}$ such that $x^{\alpha} \subseteq U$, where $x^{\alpha}$ is the open ball of radius $\alpha$ around $x$;

- The members of $\mathcal{U}$ have uniformly bounded diameters.

Recall that a cover $\mathcal{U}$ is of dimension $\leq N$ if every $x \in X$ is contained in no more then $N+1$ members of $\mathcal{U}$. The asymptotic dimension of a finitely generated group is its asymptotic dimension as a metric space with respect to any word metric. An important result of $\mathrm{Yu}$ [19] asserts that the Novikov conjecture holds for groups of finite asymptotic dimension. This can be viewed as an injectivity result for the assembly map in $L$-theory (after inverting 2 ). Further injectivity results for assembly maps for groups with finite asymptotic dimension can be found in Bartels [1], Carlsson and Goldfarb [6] and Bartels and Rosenthal [4]. On the other hand no surjectivity statement of assembly maps is known for all groups of finite asymptotic dimension and this is very much related to the absence of any equivariance condition for the cover $\mathcal{U}$ as above. 
Definition 1.1 Let $G$ be a group and $Z$ be a $G$-space. Let $\mathcal{F}$ be a collection of subgroups of $G$. An open cover $\mathcal{U}$ of $Z$ is called an $\mathcal{F}$-cover if the following two conditions are satisfied.

(i) For $g \in G$ and $U \in \mathcal{U}$ we have either $g(U)=U$ or $g(U) \cap U=\varnothing$;

(ii) For $g \in G$ and $U \in \mathcal{U}$ we have $g(U) \in \mathcal{U}$;

(iii) For $U \in \mathcal{U}$ the subgroup $G_{U}:=\{g \in G \mid g(U)=U\}$ is a member of $\mathcal{F}$.

Let $G$ be a word-hyperbolic group. Fix a set of generators $S$. Let $d_{G}$ be the word metric on $G$ with respect to $S$. Let $X$ be a hyperbolic complex with an isometric $G$-action in the sense of Mineyev [14]; see Section 6.1. Let $\partial X$ be the Gromov boundary of $X$. (This boundary can be described as a quotient of the set of geodesic rays in $X$, where two such rays are identified if they are asymptotic [5, III.H.3].) Let $\bar{X}:=X \cup \partial X$ be the compactification of $X$ [5, III.H.3]). Let $\mathcal{V C}$ yc denote the collection of virtually cyclic subgroups of $G$, that is of subgroups that have a cyclic subgroup of finite index. The following is our main result and should be thought of as an equivariant version of the (much easier) fact that hyperbolic groups have finite asymptotic dimension $[11, \mathrm{p} 31 ; 17]$.

Theorem 1.2 Let $G$ be word-hyperbolic and let $X$ be a hyperbolic complex. Suppose that there is a simplicial proper cocompact $G$-action on $X$. Equip $G \times \bar{X}$ with the diagonal $G$-action. Then there exists a natural number $N=N(G, \bar{X})$ depending only on $G$ and $\bar{X}$ such that the following holds: For every $\alpha>0$ there exists an open $\mathcal{V C}$ yc-cover $\mathcal{U}$ of $G \times \bar{X}$ satisfying

(i) $\operatorname{dim}(\mathcal{U}) \leq N$;

(ii) For $g_{0} \in G$ and $c \in \bar{X}$ there exists $U \in \mathcal{U}$ such that $g_{0}^{\alpha} \times\{c\} \subseteq U$, where $g_{0}^{\alpha}$ is the open ball with center $g_{0}$ and radius $\alpha$ with respect to the word metric $d_{G}$;

(iii) $G \backslash \mathcal{U}$ is finite.

This result plays an important role in our proof of the Farrell-Jones conjecture for $K_{*}(R G)$ for every word-hyperbolic group $G$ and every coefficient ring $R$ [3].

The conclusion of Theorem 1.2 is formally similar to the definition of finite asymptotic dimension discussed above. The price we have to pay for the equivariance of the cover $\mathcal{U}$ is the space $\bar{X}$. For the application it will be very important that $\bar{X}$ is compact. (If we replace $\bar{X}$ by a finite dimensional $G-C W$-complex all whose isotropy groups lie in $\mathcal{V}$ yc, then the conclusion follows easily from the fact that $G$ has finite asymptotic dimension.) The members of $\mathcal{U}$ are only large in the $G$-coordinate; in the $\bar{X}$-coordinate they may be very small. Similar covers have been used in a slightly different situation 
where $\bar{X}$ is replaced by a probability space with a measure preserving action of $G$; compare Gromov [12, p 300] and Sauer [18]. It would be interesting to know if there is a version of Theorem 1.2 in this situation.

It seems reasonable to hope that the class of groups $G$ for which there is a compact

$G$-space $\bar{X}$ such that the conclusion of Theorem 1.2 holds is bigger than the class of hyperbolic groups.

The proof of Theorem 1.2 is quite involved and uses a generalization of techniques used and developed by Farrell-Jones in [7]. Firstly, we study flows on metric spaces and prove the existence of long and thin covers; see Theorem 1.4. This generalizes the long and thin cells from [7, Proposition 7.2]. Secondly, we use a variant $F S(X)$ of Mineyev's half open symmetric join $* \bar{X}$ [14]. This space is a substitute for the sphere bundle of a negatively curved manifold and is equipped with a flow $\phi_{\tau}$ (corresponding to the geodesic flow on the sphere bundle). In Theorem 1.5 we improve upon Mineyev's flow estimate [14, Theorem 57 on page 468]. The required cover is then produced by pulling back a long and thin cover of $F S(X)$ by the composition of the flow $\phi_{\tau}$ for large $\tau$ with an embedding $G \times \bar{X} \rightarrow F S(X)$. A more detailed discussion follows in Sections 1.1 and 1.2.

\subsection{Long thin covers}

The existence of long thin covers will be proven in the following situation.

\section{Convention 1.3 Let}

- $G$ be a discrete group;

- $X$ be a metrizable topological space with a proper cocompact $G$-action on $X$;

- $\Phi: X \times \mathbb{R} \rightarrow X$ be a flow.

Assume that the following conditions are satisfied:

- $\Phi$ is $G$-equivariant;

- The number of closed orbits, which are not stationary and whose period is $\leq C$, of the flow induced on $G \backslash X$ is finite for every $C>0$;

- $\quad X-X^{\mathbb{R}}$ is locally connected (notation explained below);

- If we put

$$
\begin{aligned}
& k_{G}:=\sup \{|H| \mid H \subseteq G \text { subgroup with finite order }|H|\}, \\
& d_{X}:=\operatorname{dim}\left(X-X^{\mathbb{R}}\right),
\end{aligned}
$$

then $k_{G}<\infty$ and $d_{X}<\infty$. 
Recall that a $G$-action is proper if for every $x \in X$ there exists an open neighborhood $U$ such that the set $\{g \in G \mid U \cap g U \neq \varnothing\}$ is finite. Recall that $X$ is locally connected if for each $x \in X$ and each open neighborhood $U$ of $x$ we can find a connected open neighborhood $U^{\prime}$ of $x$ with $U^{\prime} \subseteq U$. A $G$-space $X$ is called cocompact if $G \backslash X$ is compact. The dimension of a collection of subsets $\left\{U_{i} \mid i \in I\right\}$ is $\leq d$, if every point is contained in at most $d+1$ members of the $U_{i}$. The covering dimension of a space $X$ is $\leq d$ if every open covering has an open refinement whose dimension is less or equal to $d$. One may replace the covering dimension $d_{X}$ of $X-X^{\mathbb{R}}$ appearing above by the supremum of the covering dimensions of compact subsets of $X-X^{\mathbb{R}}$. Recall that an equivariant flow $\Phi: \mathbb{R} \times X \rightarrow X$ is a continuous $\mathbb{R}$-action, such that $\Phi_{\tau}(g x)=g \Phi_{\tau}(x)$ holds for all $g \in G, \tau \in \mathbb{R}$ and $x \in X$. We denote by $X^{\mathbb{R}}$ the $\mathbb{R}$-fixed point set, ie, the set of points $x \in X$ for which $\Phi_{\tau}(x)=x$ for all $\tau \in \mathbb{R}$. The period of a closed orbit of $\Phi$ which is not stationary is the smallest number $\tau>0$ such that $\Phi_{\tau}(x)=x$ holds for all $x$ in this orbit.

The following is our main result in this situation.

Theorem 1.4 There exists a natural number $N$ depending only on $k_{G}, d_{X}$ and the action of $G$ on an arbitrary small neighborhood of $X^{\mathbb{R}}$ such that for every $\alpha>0$ there is an $\mathcal{V C} y c-c o v e r \mathcal{U}$ of $X$ with the following two properties:

(i) $\operatorname{dim} \mathcal{U} \leq N$;

(ii) For every $x \in X$ there exists $U \in \mathcal{U}$ such that

$$
\Phi_{[-\alpha, \alpha]}(x):=\left\{\Phi_{\tau}(x) \mid \tau \in[-\alpha, \alpha]\right\} \subseteq U
$$

(iii) $G \backslash \mathcal{U}$ is finite.

The main difference between Theorem 1.4 and [7, Proposition 7.2] is that we deal with metric spaces rather than manifolds. This requires a different type of general position argument (compare Section 3) and forces us to work with open covers rather than cell structures. While cell structures of a manifold are automatically finite dimensional, in our situation more care is needed to establish the bound on the dimension of $\mathcal{U}$ and our bound is much larger then the dimension of the metric space $X$. Finally, we deal with an honest proper action and do not require a torsion free subgroup of finite index, as is used in [8].

The proof of Theorem 1.4 will be given in Section 5 and depends on Sections 2, 3 and 4 . 


\subsection{The flow space}

Let $G$ be a hyperbolic group. Fix a set of generators $S$. Let $d_{G}$ be the word metric on $G$ with respect to $S$. Let $X$ be a hyperbolic complex and $\bar{X}=X \cup \partial X$ be its compactification as before. Assume that $G$ acts isometrically on $X$. In Section 6 we introduce the metric space $\left(F S(X), d_{F S}\right)$. This space is equipped with an isometric $G$-action and a $G$-equivariant flow $\phi_{\tau}$.

Our main flow estimate is the following.

Theorem 1.5 There exists a continuous $G$-equivariant (with respect to the diagonal $G$-action on the source) map $j: G \times \bar{X} \rightarrow F S(X)$ such that for every $\alpha>0$ there exists a number $\beta=\beta(\alpha)$ such that the following holds:

If $g, h \in G$ with $d_{G}(g, h) \leq \alpha$ and $c \in \bar{X}$ then there is $\tau_{0} \in \mathbb{R}$ with $\left|\tau_{0}\right| \leq \beta$ such that for all $\tau \in \mathbb{R}$

$$
d_{F S}\left(\phi_{\tau} j(g, c), \phi_{\tau+\tau_{0}} j(h, c)\right) \leq f_{\alpha}(\tau) \text {. }
$$

Here $f_{\alpha}: \mathbb{R} \rightarrow[0, \infty)$ is a function that depends only on $\alpha$ and has the property that $\lim _{\tau \rightarrow \infty} f_{\alpha}(\tau)=0$.

An important ingredient of the proof of this result is Theorem 7.1 which is an improvement of Mineyev's [14, Theorem 57 on page 468]. The main differences are that we consider points not necessary on the same horosphere, and that we consider the action of the flow $\phi_{\tau}$ and not translation by length. In addition, Mineyev's estimate is in terms of a pseudo-metric, not in terms of the metric $d_{F S}$.

In order to apply Theorem 1.4 to $F S(X)$ we need further properties of the flow space and $G$.

\section{Proposition 1.6}

(i) The order of finite subgroups in $G$ is bounded.

(ii) $F S(X)-F S(X)^{\mathbb{R}}$ is locally connected and has finite covering dimension.

(iii) If the action of $G$ on $X$ is cocompact and proper, then action of $G$ on $F S(X)$ is also cocompact and proper.

(iv) If the action of $G$ on $X$ is cocompact and proper, then the number of closed orbits, which are not stationary and whose period is $\leq C$, of the flow induced on $G \backslash F S(X)$ is finite for every $C>0$.

The proof of Theorem 1.5 will be given in Section 8 and depends only on Sections 6 and 7. The proof of Proposition 1.6 will be given in Section 9 and depends only on Sections 2 and 6. 


\subsection{Construction of the cover}

Using the results from Sections 1.1 and 1.2 we can now give the proof of Theorem 1.2. During this proof we will use the following notation: if $A$ is a subset of a metric space $Z$ and $\delta>0$, then $A^{\delta}$ denotes the set of all points $z \in Z$ for which $d(z, A)<\delta$; compare Definition 3.1.

Proof Consider any $\alpha>0$. Let $\beta=\beta(\alpha)$ be the number appearing in Theorem 1.5. It follows from Proposition 1.6 that Theorem 1.4 can be applied to $F S(X)$. Thus there is a number $N$ (independent of $\alpha$ ) such that there exists an $\mathcal{V C}$ yc-cover $\mathcal{V}$ of $F S(X)$ of dimension no more than $N$ with the following property: For every $\xi \in F S(X)$ there exists $V_{\xi} \in \mathcal{V}$ such that

$$
\phi_{[-2 \beta, 2 \beta]}(\xi)=\left\{\phi_{\tau}(\xi) \mid \tau \in[-2 \beta, 2 \beta]\right\} \subseteq V_{\xi} .
$$

Since $\phi_{[-2 \beta, 2 \beta]}(\xi)$ is compact, $V_{\xi}$ is open and $\phi_{[-2 \beta, 2 \beta]}(\xi) \subseteq V_{\xi}$, we can find $\delta_{\xi}>0$ (depending on $\xi$ and $\beta, V_{\xi}$ ) such that

$$
\left(\phi_{[-2 \beta, 2 \beta]}(\xi)\right)^{\delta_{\xi}} \subseteq V_{\xi} .
$$

Because $G$ acts by isometries, we can arrange that $\delta_{\xi}=\delta_{g \xi}$ holds for all $g \in G$. In particular we get $g \cdot\left(\phi_{[-2 \beta, 2 \beta]}(\xi)\right)^{\delta_{\xi}}=\left(\phi_{[-2 \beta, 2 \beta]}(g \xi)\right)^{\delta_{g \xi}}$. For $\xi \in F S(X)$ pick $\epsilon_{\xi}>0$ such that

$$
0<e^{\beta} \epsilon_{\xi}<\delta_{\xi} / 2 .
$$

Again we arrange that $\epsilon_{g \xi}=\epsilon_{\xi}$ holds for all $g \in G$. Obviously the collection

$$
\left\{\left(\phi_{[-\beta, \beta]}(\xi)\right)^{\epsilon \xi} \mid \xi \in F S(X)\right\}
$$

is an open covering of $F S(X)$. Since $G$ acts cocompactly, we can find finitely many points $\xi_{i}$ for $i=0,1,2, \ldots, I$ for some positive natural number $I$ such that the $G$-cofinite collection

$$
\left\{\left(\phi_{[-\beta, \beta]}\left(g \xi_{i}\right)\right)^{\epsilon_{g \xi_{i}}} \mid g \in G, i \in\{0,1,2 \ldots, I\}\right\}
$$

is an open covering of $F S(X)$. Consider $\xi \in F S(X)$. Then we can find $i=i(\xi) \in$ $\{0,1,2 \ldots, I\}$ and $g=g(\xi) \in G$ such that $\xi \in\left(\phi_{[-\beta, \beta]}\left(g \xi_{i}\right)\right)^{\epsilon_{g \xi_{i}}}$. In particular, there is $\tau \in[-\beta, \beta]$ such that $d_{F S}\left(\xi, \phi_{\tau}\left(g \xi_{i}\right)\right)<\epsilon_{g \xi_{i}}$. Let

$$
\delta:=\min \left\{\delta_{\xi_{i}} / 2 \mid i=0,1,2 \ldots, I\right\} .
$$

Consider $\zeta \in\left(\phi_{[-\beta, \beta]}(\xi)\right)^{\delta}$. Choose $\sigma \in[-\beta, \beta]$ satisfying $d_{F S}\left(\zeta, \phi_{\sigma}(\xi)\right)<\delta$. In the following estimate we will use Lemma 7.2. (In this lemma the more careful notation 
$d_{F S, x_{0}}$ is used for $d_{F S}$.)

$$
\begin{aligned}
d_{F S}\left(\zeta, \phi_{\sigma+\tau}\left(g \xi_{i}\right)\right) & \leq d_{F S}\left(\zeta, \phi_{\sigma}(\xi)\right)+d_{F S}\left(\phi_{\sigma}(\xi), \phi_{\sigma+\tau}\left(g \xi_{i}\right)\right) \\
& <\delta+e^{|\sigma|} \cdot d_{F S}\left(\xi, \phi_{\tau}\left(g \xi_{i}\right)\right) \\
& <\delta+e^{\beta} \cdot \epsilon_{g \xi_{i}} \\
& <\delta_{g \xi_{i}}
\end{aligned}
$$

Since $\sigma+\tau \in[-2 \beta, 2 \beta]$, this implies

$$
\left(\phi_{[-\beta, \beta]}(\xi)\right)^{\delta} \subseteq\left(\phi_{[-2 \beta, 2 \beta]}\left(g \xi_{i}\right)\right)^{\delta_{g \xi_{i}}} \subseteq V_{g \xi_{i}} .
$$

Thus we have found $\delta>0$ such that for every $\xi \in F S(X)$ there exists $V_{\xi} \in \mathcal{V}$ such that

$$
\left(\phi_{[-\beta, \beta]}(\xi)\right)^{\delta} \subseteq V_{\xi}
$$

We will construct the desired open covering $\mathcal{U}$ of $G \times \bar{X}$ by pulling back $\mathcal{V}$ with the composition

$$
G \times \bar{X} \stackrel{j}{\rightarrow} F S(X) \stackrel{\phi_{\tau}}{\longrightarrow} F S(X)
$$

for an appropriate real number $\tau$, where $j$ is the map from Theorem 1.5. Obviously $\mathcal{U}$ has for every choice of $\tau$ all the desired properties except for the property that there exists $U_{\left(g_{0}, c\right)} \in \mathcal{U}$ such that $g_{0}^{\alpha} \times\{c\} \subseteq U_{\left(g_{0}, c\right)}$ for every $c \in \bar{X}$ and every $g_{0} \in G$.

We conclude from Theorem 1.5 for $\tau \in \mathbb{R}$ and the function $f_{\alpha}$ appearing in Theorem 1.5

$$
\phi_{\tau} \circ j(g, c) \in\left(\phi_{[-\beta, \beta]}\left(\phi_{\tau} \circ j\left(g_{0}, c\right)\right)\right)^{f_{\alpha}(\tau)}
$$

for all $c \in \bar{X}$ and all $g \in G$ with $d_{G}\left(g_{0}, g\right)<\alpha$. By Theorem 1.5 there is $\tau$ such that $f_{\alpha}(\tau)<\delta$. For such a choice of $\tau$ we conclude from (1.7) that

$$
\phi_{\tau} \circ j(g, c) \in\left(\phi_{[-\beta, \beta]}\left(\phi_{\tau} \circ j\left(g_{0}, c\right)\right)\right)^{\delta} \subset V_{\phi_{\tau} \circ j\left(g_{0}, c\right)}
$$

for all $c \in \bar{X}$ and all $g \in G$ with $d_{G}\left(g, g_{0}\right)<\alpha$. This finishes the proof of Theorem 1.2 .

\section{Acknowledgements}

We thank Igor Mineyev for useful conversations on his paper and helpful comments on a preliminary version of Sections 6 to 9. We thank Tom Farrell who a long time ago explained the proof of [7, Proposition 7.2] to us. Moreover we are grateful to the referee who read through the manuscript very carefully and made a lot of helpful comments. 


\section{Boxes}

Convention 2.1 Throughout this section we consider

- a discrete group $G$;

- a metrizable topological space $X$;

- a proper cocompact $G$-action on $X$;

- a $G$-equivariant flow $\Phi: X \times \mathbb{R} \rightarrow X$ such that $X-X^{\mathbb{R}}$ is locally connected.

\subsection{Basics about boxes}

In this subsection we introduce and study the notion of a box.

Definition 2.2 Let $B$ be a subset of a $G$-space. Define a subgroup of $G$ by

$$
G_{B}:=\{g \in G \mid g B=B\},
$$

where $g B:=\{g b \mid b \in B\}$.

A subset $B$ of a topological $G$-space is called an $\mathcal{F}$-subset for a collection $\mathcal{F}$ of subgroups of $G$, if $G_{B}$ belongs to $\mathcal{F}$ and for all $g \in G$ we have the implication $g B \cap B \neq \varnothing \Rightarrow B=g B$.

Notice that $g B=B$ does not imply that $g b=b$ holds for all $b \in B$. We denote by $\mathcal{F}$ in the collection of finite subgroups.

Definition 2.3 A box $B$ is a subset $B \subseteq X$ with the following properties:

(i) $B$ is a compact $\mathcal{F}$ in-subset;

(ii) There exists a real number $l=l_{B}>0$, called the length of the box $B$, with the property that for every $x \in B$ there exists real numbers $a_{-}(x) \leq 0 \leq a_{+}(x)$ and $\epsilon(x)>0$ satisfying

$$
\begin{aligned}
l & =a_{+}(x)-a_{-}(x) \\
\Phi_{\tau}(x) & \in B \text { for } \tau \in\left[a_{-}(x), a_{+}(x)\right] \\
\Phi_{\tau}(x) & \notin B \text { for } \tau \in\left(a_{-}(x)-\epsilon(x), a_{-}(x)\right) \cup\left(a_{+}(x), a_{+}(x)+\epsilon(x)\right) .
\end{aligned}
$$

Definition 2.4 Let $B \subseteq X$ be a box. Then the following data are associated to it:

- The length $l_{B}>0$;

- Let $G_{B} \subseteq G$ be the finite subgroup $\{g \in G \mid g B=B\}$; 
- We denote by $B^{\circ}$ the (topological) interior and by $\partial B$ the (topological) boundary of $B \subseteq X$;

- Let $S_{B} \subseteq B$ be the set of points $\left\{x \in B \mid a_{-}(x)+a_{+}(x)=0\right\}$. We call $S_{B}$ the central slice of $B$;

- Let $\partial_{ \pm} B$ be the set of points $\left\{x \in B \mid a_{ \pm}(x)=0\right\}=\left\{\phi_{a_{ \pm}(x)}(x) \mid x \in S_{B}\right\}$. We call $\partial_{-} B$ the bottom and $\partial_{+} B$ the top of $B$. Define the open bottom and open top $\partial_{ \pm} B^{\circ}:=\left\{\phi_{a_{ \pm}(x)}(x) \mid x \in S_{B} \cap B^{\circ}\right\}$;

- Let $\pi_{B}: B \rightarrow S_{B}$ be the retraction onto the central slice which sends $x$ to $\Phi_{\left(a_{+}(x)+a_{-}(x)\right) / 2}(x)$.

Remark 2.5 A box does not intersect $X^{\mathbb{R}}$ but may intersect a closed orbit. A box does never contain a closed orbit. It may happen that a nonclosed orbit meets the central slice infinitely many times, but whenever it meets the central slice it has to leave the box before it comes back to the central slice. We do not require that the central slice is connected. We have for $x \in B, \tau \in\left[a_{-}(x), a_{+}(x)\right]$ that $\Phi_{\tau}(x) \in B$ and

$$
\begin{aligned}
& a_{-}\left(\Phi_{\tau}(x)\right)=a_{-}(x)-\tau ; \\
& a_{+}\left(\Phi_{\tau}(x)\right)=a_{+}(x)-\tau .
\end{aligned}
$$

Lemma 2.6 Let $B \subseteq X$ be a box of length $l=l_{B}$. Then the following holds:

(i) We get for $g \in G_{B}$ and $x \in X$

$$
\begin{aligned}
& a_{-}(g x)=a_{-}(x) ; \\
& a_{+}(g x)=a_{+}(x) .
\end{aligned}
$$

The bottom $\partial_{-} B$, the open bottom $\partial_{-} B^{\circ}$, the top $\partial_{+} B$, the open top $\partial_{+} D^{\circ}$, the central slice $S_{B}$ and the interior $B^{\circ}$ are $\mathcal{F}$ in-subsets of $G$ and satisfy (unless they are empty)

$$
G_{B}=G_{B^{\circ}}=G_{\partial_{-} B}=G_{\partial_{-} B^{\circ}}=G_{\partial_{+} B}=G_{\partial_{+} B^{\circ}}=G_{S_{B}} ;
$$

(ii) The maps

$$
a_{ \pm}: B \rightarrow \mathbb{R}, \quad x \mapsto a_{ \pm}(x)
$$

are continuous;

(iii) The maps

$$
\begin{gathered}
\mu: S_{B} \times[-l / 2, l / 2] \stackrel{\cong}{\rightarrow} B, \quad(x, \tau) \mapsto \Phi_{\tau}(x) \\
\text { and } \mu^{-1}: B \stackrel{\cong}{\rightarrow} S_{B} \times[-l / 2, l / 2], \quad x \mapsto\left(\Phi_{\left.\frac{a_{-(x)+a_{+}(x)}}{2}(x), l / 2-a_{+}(x)\right)}\right.
\end{gathered}
$$


are to one another inverse $G_{B}$-homeomorphisms, where $G_{B}=G_{S_{B}}$ acts on $S_{B} \times[-l / 2, l / 2]$ by $g \cdot(s, t)=(g s, t)$.

We have

$$
\begin{aligned}
& B^{\circ}=\mu\left(\left(S_{B} \cap B^{\circ}\right) \times(-l / 2, l / 2)\right) ; \\
& \partial B=\mu\left(\left(S_{B} \cap \partial B\right) \times[-l / 2, l / 2] \cup\left(S_{B} \times\{-l / 2, l / 2\}\right)\right) ; \\
& \partial_{ \pm} B=\mu\left(S_{B} \times\{ \pm l / 2\}\right) ; \\
& \partial_{ \pm} B^{\circ}=\mu\left(\left(S_{B} \cap B^{\circ}\right) \times\{ \pm l / 2\}\right) ;
\end{aligned}
$$

(iv) The space $S_{B} \cap B^{\circ}$ is locally connected;

(v) There exists $\epsilon_{B}>0$ depending only on $B$ such that the numbers $\epsilon(x)$ appearing in Definition 2.3 can be chosen so that $\epsilon(x) \geq \epsilon_{B}$ holds for all $x \in B$.

Proof (i) For $x \in B$ and $g \in G_{B}$ we have $\Phi_{\tau}(x) \in B \Leftrightarrow g \cdot \Phi_{\tau}(x)=\Phi_{\tau}(g x) \in B$. This implies $a_{ \pm}(g x)=a_{ \pm}(x)$ for $x \in B$ and $g \in G_{B}$. We conclude from the definition of the bottom $\partial_{-} B$, the open bottom $\partial_{-} B^{\circ}$, the top $\partial_{+} B$, the open top $\partial_{+} B^{\circ}$, the central slice $S_{B}$ and the interior $B^{\circ}$ that these sets are $G_{B}$ invariant and contained in the $\mathcal{F}$ in-subset $B$. Hence they are themselves $\mathcal{F}$ in-subsets of $X$ and satisfy $G_{B}=G_{B^{\circ}}=G_{\partial_{-} B}=G_{\partial_{+} B}=G_{S_{B}}$ if nonempty.

(ii) Consider $x \in B$ and $\epsilon>0$ with $\epsilon<\epsilon(x)$, where $\epsilon(x)$ is the number appearing in Definition 2.3. The points $\Phi_{a_{ \pm}(x) \pm \epsilon}(x)$ lie outside $B$. Since $B$ is compact and $X$ is a Hausdorff space, we can find an open neighborhood $V_{ \pm}$of $\Phi_{a_{ \pm}(x) \pm \epsilon}(x)$ such that $V_{ \pm}$does not meet $B$. Put $U_{ \pm}=\left(\Phi_{a_{ \pm}}(x) \pm \epsilon\right)^{-1}\left(V_{ \pm}\right)$. Then $x \in U_{ \pm}$and $\Phi_{a_{ \pm}(x) \pm \epsilon}(u)$ does not lie in $B$ for $u \in U_{ \pm}$. This implies $a_{+}(u)<a_{+}(x)+\epsilon$ for $u \in U_{+} \cap B$ and $a_{-}(x)-\epsilon<a_{-}(u)$ for $u \in U_{-} \cap B$. Put $U=U_{-} \cap U_{+} \cap B$. Then $U \subseteq B$ is an open neighborhood of $x$ in $B$ such that $a_{-}(x)-\epsilon<a_{-}(u)$ and $a_{+}(u)<a_{+}(x)+\epsilon$ holds for $u \in U$. Since $a_{+}(u)-a_{-}(u)=l$ for all $u \in U$, we conclude $a_{ \pm}(u) \in\left(a_{ \pm}(x)-\epsilon, a_{ \pm}(x)+\epsilon\right)$ for all $u \in U$. Hence $a_{ \pm}$is continuous.

(iii) The maps $\mu$ and $\mu^{-1}$ are continuous since $\Phi$ and by assertion (ii) the maps $a_{+}$ and $a_{-}$are continuous. One easily checks that they are inverse to one another.

Since the flow is compatible with the $G$-action and $G_{B}=G_{S_{B}}$, the map $\mu$ is $G_{S_{B}}=$ $G_{B}$-equivariant.

Next we prove

$$
\begin{array}{r}
\mu\left(\left(S_{B} \cap B^{\circ}\right) \times(-l / 2, l / 2)\right) \subseteq B^{\circ} ; \\
\mu\left(\left(S_{B} \cap \partial B\right) \times[-l / 2, l / 2] \cup\left(S_{B} \times\{-l / 2, l / 2\}\right)\right) \subseteq \partial B .
\end{array}
$$

Consider $(x, \tau) \in\left(S_{B} \cap B^{\circ}\right) \times(-l / 2, l / 2)$. Since $a_{-}$and $a_{+}$are continuous by assertion (ii) and $a_{-}(x)=-l / 2$ and $a_{+}(x)=l / 2$, we can find an open neighborhood 
$U \subseteq B^{\circ}$ of $x$ such that $\tau \in\left(a_{-}(u), a_{+}(u)\right)$ holds for all $u \in U$. Hence $\Phi_{\tau}(U)$ is contained in $B$. Since $\Phi_{\tau}(U)$ is an open subset of $X$, we have $\Phi_{\tau}(U) \subseteq B^{\circ}$. Since $\mu(x, \tau)=\Phi_{\tau}(x)$ lies in $\Phi_{\tau}(U)$, the inclusion (2.7) is proven.

Consider $x \in S_{B}$. Let $U \subseteq X$ be an open neighborhood of $\Phi_{l / 2}(x)$. Since $\mathbb{R} \rightarrow$ $X, \tau \mapsto \Phi_{\tau}(x)$ is a continuous map, there is an $\epsilon$ with $0<\epsilon<l / 2$ such that $\Phi_{\tau}(x) \in U$ holds for $\tau \in(l / 2-\epsilon, l / 2+\epsilon)$. Since $\left\{\Phi_{\tau}(x) \mid \tau \in(l / 2-\epsilon, l / 2)\right\}$ is contained in $B$ and $\left\{\Phi_{\tau}(x) \mid \tau \in(l / 2, l / 2+\epsilon)\right\}$ is contained in $X-B$, the open neighborhood $U$ of $\Phi_{l / 2}(x)$ intersects both $B$ and $X-B$. This shows $\Phi_{l / 2}(x)=\mu(x, l / 2) \in \partial B$. Analogously one proves $\Phi_{-l / 2}(x)=\mu(x,-l / 2) \in \partial B$.

Consider $x \in S_{B} \cap \partial B$ and $\tau \in(-l / 2, l / 2)$. We want to show $\mu(x, \tau) \in \partial B$. Suppose the converse. Since $\mu(x, \tau)=\Phi_{\tau}(x)$ belongs to $B$, there must be an open neighborhood $U$ of $\Phi_{\tau}(x)$ such that $U \subseteq B$. Since the functions $a_{-}$and $a_{+}$are continuous by assertion (ii) and $a_{-}(x)=-l / 2<-\tau<a_{+}(x)=l / 2$, we can arrange by making $U$ smaller that $-\tau \in\left(a_{-}(u), a_{+}(u)\right)$ holds for all $u \in U$. Hence $\Phi_{-\tau}(U)$ is an open subset of $X$ which is contained in $B$ and contains $x$. This contradicts $x \in \partial B$. This finishes the proof of (2.8). Now assertion (iii) follows from (2.7) and (2.8).

(iv) Since $B^{\circ}$ is an open subset of the locally connected space $X-X^{\mathbb{R}}$, it is itself locally connected. Because of assertion (iii) the space $S_{B} \cap B^{\circ} \times(-l / 2, l / 2)$ is locally connected. Since the projection $S_{B} \cap B^{\circ} \times(-l / 2, l / 2) \rightarrow S_{B} \cap B^{\circ}$ is an open continuous map and the image of a connected set under a continuous maps is again connected, $S_{B} \cap B^{\circ}$ is locally connected.

(v) Suppose that such $\epsilon_{B}$ does not exists. Then we can find a sequence $\left(x_{n}\right)_{n \geq 0}$ of elements in $B$ and a sequence $\left(\tau_{n}\right)_{n \geq 0}>0$ of positive real numbers with $\lim _{n \rightarrow \infty} \tau_{n}=0$ such that one of the following holds for $n \geq 0$ :

(a) $\Phi_{a_{-}\left(x_{n}\right)-\tau_{n}}\left(x_{n}\right) \in B$ and $\Phi_{a_{-}\left(x_{n}\right)-\tau}\left(x_{n}\right) \notin B$ for $\tau \in\left(0, \tau_{n}\right)$

(b) $\Phi_{a_{+}\left(x_{n}\right)+\tau_{n}}\left(x_{n}\right) \in B$ and $\Phi_{a_{+}\left(x_{n}\right)+\tau}\left(x_{n}\right) \notin B$ for $\tau \in\left(0, \tau_{n}\right)$

By passing to a subsequence we can arrange that $x_{n}$ converges to some point $x \in B$ and (a) holds for all $n \geq 0$ or (b) holds for all $n \geq 0$. We only treat the case (a), where $\Phi_{a_{-}\left(x_{n}\right)-\tau_{n}}\left(x_{n}\right) \in B$ and $\Phi_{a_{-}\left(x_{n}\right)-\tau}\left(x_{n}\right) \notin B$ for $\tau \in\left(0, \tau_{n}\right)$ holds for all $n \geq 0$, the proof in the other case (b) is analogous. Put $y_{n}=\Phi_{a_{-}\left(x_{n}\right)-\tau_{n}}\left(x_{n}\right)$. Then $y_{n} \in \partial_{+} B$ for all $n \geq 0$ since $y_{n} \in B$ and $\Phi_{\tau}\left(y_{n}\right)=\Phi_{a_{-}\left(x_{n}\right)-\tau_{n}+\tau}\left(x_{n}\right) \notin B$ holds for $\tau \in\left(0, \tau_{n}\right)$. We conclude $\lim _{n \rightarrow \infty} a_{-}\left(x_{n}\right)=a_{-}(x)$ from assertion (ii). Hence $\lim _{n \rightarrow \infty} y_{n}=\Phi_{a_{-}(x)}(x)$. Since $y_{n} \in \partial_{+} B$ for $n \geq 0$, we have $\lim _{n \rightarrow \infty} y_{n} \in \partial_{+} B$. This contradicts $\Phi_{a_{-}(x)}(x) \in \partial_{-} B$ since $l_{B}>0$.

We mention that in general $S_{B}$ itself is not locally connected. 
Remark 2.9 It is a little bit surprising that the function $a_{ \pm}$is continuous as stated in Lemma 2.6 (ii) since there seem to be no link between different flow lines entering the box. The point here is that we require the box to be compact. If $G$ is trivial and we consider the flow $\phi_{\tau}(x, y)=(x+\tau, y)$ on $\mathbb{R}^{2}$, the subset of $\mathbb{R}^{2}$ given by

$$
B:=\{(x, y) \mid x, y \in[-1,1], y \neq 0\} \cup\{(x, 0) \mid x \in[0,2]\}
$$

satisfies all the requirements of a box of length 2 except for compactness and the functions $a_{ \pm}$are not continuous at $(0,0)$.

Definition 2.10 Consider a box $B$ of length $l_{B}$. Let $V \subseteq S_{B}$ be a nonempty closed $\mathcal{F}$ in-subset of the $G_{S_{B}}$-space $S_{B}$ and $a, b$ real numbers with $-l_{B} / 2 \leq a<b \leq l_{B} / 2$. Define a new box of length $b-a$ by

$$
B(V ; a, b):=\Phi_{[a, b]}(V):=\left\{\Phi_{\tau}(v) \mid v \in V, \tau \in[a, b]\right\} .
$$

If $a=-v / 2$ and $b=v / 2$ for some $v \in[0, w]$ we abbreviate

$$
B(V ; v):=B(V ;-v / 2, v / 2) .
$$

If $a=-l_{B} / 2$ and $b=l_{B} / 2$, we abbreviate

$$
B(V):=B\left(V ;-l_{B} / 2, l_{B} / 2\right),
$$

and call $B(V)$ the restriction of $B$ to $V$.

We have to show that $B(V ; a, b)$ is again a box. Since $V$ is a closed subset of the compact set $S_{A}$, it is compact. Hence $V \times[a, b]$ is compact. We conclude that $B(V, a, b)$ as the image of a compact set under the continuous map $\Phi: X \times \mathbb{R} \rightarrow X$ is compact. From Lemma 2.6 we get $G_{B}=G_{S_{B}}$ and the $G_{B}$-equivariant homeomorphism

$$
\mu_{B}: S_{B} \times\left[-l_{B} / 2, l_{B} / 2\right] \stackrel{\cong}{\rightarrow} B, \quad(x, \tau) \mapsto \Phi_{\tau}(x) .
$$

The subset $V$ of the $G_{B}$-space $S_{B}$ is a $\mathcal{F}$ in-subset. Hence $B(V ; a, b)=\mu_{B}(V \times[a, b])$ is a $\mathcal{F}$ in-subset of the $G_{B}$-space $B$. Since $B$ is a $\mathcal{F}$ in-subset of the $G$-space $X$, $B(V ; a, b)$ is a $\mathcal{F}$ in-subset of the $G$-space $X$. Consider $x \in B(V ; a, b)$. We can write it as $x=\Phi_{\tau}(v)$ for $v \in S_{B}$ and $\tau \in[a, b]$. Put $a_{-}(x)=a-\tau$ and $a_{+}(x)=b-\tau$. Let $\epsilon(x)$ for $x \in B$ be the number appearing in the Definition 2.3 of a box for $B$. Now one easily checks that the collections $a_{ \pm}(x)$ and $\epsilon(x)$ have the desired properties of 
Definition 2.3 for $B(V ; a, b)$. This shows that $B(V ; a, b)$ is a box. We have

$$
\begin{aligned}
\partial_{-} B(V ; a, b) & =\Phi_{a}(V) ; \\
\partial_{+} B(V ; a, b) & =\Phi_{b}(V) ; \\
\partial_{-} B(V ; a, b)^{\circ} & =\Phi_{a}\left(V \cap B^{\circ}\right) ; \\
\partial_{+} B(V ; a, b) & =\Phi_{b}\left(V \cap B^{\circ}\right) ; \\
S_{B(V ; a, b)} & =\Phi_{\frac{a+b}{2}}(V) .
\end{aligned}
$$

In particular $B(V ; v)$ is a box of length $v$ with central slice $V$ and $B(V)$ is box of length $l_{B}$ with central slice $V$.

\subsection{Constructing boxes}

Lemma 2.11 For every $x \in X-X^{\mathbb{R}}$ there exists a nonequivariant box whose interior contains $x$.

The following proof is a variation of an argument used in [16, Theorem 1.2.7].

Proof of Lemma 2.11 Because the $G$-action on the metrizable space $X$ is proper and cocompact $X$ is locally compact; compare [13, Theorem 1.38 on page 27]. Let $\alpha>0$ with $\Phi_{\alpha}(x) \neq x$. Let $W_{ \pm}$be a closed neighborhood of $\Phi_{ \pm \alpha}(x)$ that does not contain $x$. By continuity there exist compact neighborhoods $U^{\prime} \subset U$ of $x$ and $\epsilon>0$ such that $\Phi_{[ \pm \alpha-\epsilon, \pm \alpha+\epsilon]} U \subset W_{ \pm}$and $\Phi_{[-\epsilon, \epsilon]} U^{\prime} \subset U$ and $U$ is disjoint from $W_{-} \cup W_{+}$. Let $f: X \rightarrow[0, \infty)$ be a continuous function with $f(y)=1$ for $y \in U$ and $f(y)=0$ for $y \in W_{-} \cup W_{+}$. Define $\psi: U \rightarrow \mathbb{R}$ by

$$
\psi(y)=\ln \left(\int_{-\alpha}^{\alpha} f\left(\Phi_{\tau}(y)\right) e^{-\tau} d \tau\right) .
$$

(The logarithm makes sense because the integrant is nonnegative, continuous, and positive for $\tau=0$.) Let $y \in U^{\prime}$. If $s \in[ \pm \alpha-\epsilon, \pm \alpha+\epsilon]$ then $\Phi_{s}(y) \in W_{ \pm}$and therefore $f\left(\Phi_{s}(y)\right)=0$. Using this we compute for $\delta \in[-\epsilon, \epsilon]$

$$
\begin{aligned}
\psi\left(\Phi_{\delta}(y)\right) & =\ln \left(\int_{-\alpha}^{\alpha} f\left(\Phi_{\tau+\delta}(y)\right) e^{-\tau} d \tau\right) \\
& =\ln \left(\int_{-\alpha+\delta}^{\alpha+\delta} f\left(\Phi_{\tau}(y)\right) e^{-\tau+\delta} d \tau\right) \\
& =\ln \left(e^{\delta} \int_{-\alpha+\delta}^{\alpha+\delta} f\left(\Phi_{\tau}(y)\right) e^{-\tau} d \tau\right)
\end{aligned}
$$

Geometry 83 Topology, Volume 12 (2008) 


$$
\begin{aligned}
& =\delta+\ln \left(\int_{-\alpha}^{\alpha} f\left(\Phi_{\tau}(y)\right) e^{-\tau} d \tau\right) \\
& =\delta+\psi(y) .
\end{aligned}
$$

Define $S:=U^{\prime} \cap \psi^{-1}(\psi(x))$. Then $B:=\left\{\Phi_{\tau}(s) \mid s \in S, \tau \in[-\epsilon / 2, \epsilon / 2]\right\}$ is a box whose interior contains $x$.

Definition 2.12 Let $C$ be a box of length $l_{C}$. Let

$$
\mu_{C}: S_{C} \times\left[-l_{C} / 2, l_{C} / 2\right] \rightarrow C, \quad(x, \tau) \mapsto \Phi_{\tau}(x)
$$

be the homeomorphism appearing in Lemma 2.6 (iii).

Consider a subset $S \subseteq C$. It is called transversal to the flow with respect to $C$ if $\mu_{C}^{-1}(S) \cap\{x\} \times\left[-l_{C} / 2, l_{C} / 2\right]$ consists of at most one point for every $x \in S_{C}$.

Lemma 2.13 Let $C$ be box of length $l_{C}$. Let $B$ be a box with $B \subseteq C$. Then we can find for every $x \in S_{B}$ a closed neighborhood $U \subseteq S_{B}$ of $x$ satisfying

(i) $U$ is a $G_{x}$-invariant $\mathcal{F}$ in-subset of the $G_{B}$-space $S_{B}$;

(ii) $U$ is transversal to the flow with respect to $C$.

Proof Let $\tau_{S_{B}}: S_{B} \rightarrow\left[-l_{C} / 2, l_{C} / 2\right]$ be the continuous function given by the restriction to $S_{B}$ of the composite of the projection $S_{C} \times\left[-l_{C} / 2, l_{C} / 2\right] \rightarrow\left[-l_{C} / 2, l_{C} / 2\right]$ and $\mu_{C}^{-1}$. Let $U_{1} \subseteq S_{B}$ be a closed neighborhood of $x \in S_{B}$ such that $\left|\tau_{S_{B}}(u)-\tau_{S_{B}}(x)\right|<$ $l_{B} / 2$ holds for $u \in U_{1}$. Choose a closed neighborhood $U_{2} \subseteq S_{B}$ of $x$ such that $g U_{2} \cap U_{2} \neq \varnothing \Rightarrow g \in G_{x}$ holds for $g \in G_{B}$. Put $U=\bigcap_{g \in G_{x}} g\left(U_{1} \cap U_{2}\right)$. This is a closed neighborhood of $x$ in $S_{B}$ which is $G_{x}$-invariant, a $\mathcal{F}$ in-subset of the $G_{B}$-space $S_{B}$ and satisfies $\left|\tau_{S_{B}}(u)-\tau_{S_{B}}(x)\right| \leq l_{B} / 2$ for $u \in U$.

It remains to show that $U$ is transversal to the flow with respect to $C$. Suppose the converse. So we can find $u_{0}, u_{1} \in U, v \in S_{C}$ and $\tau_{0}, \tau_{1}$ such that $u_{0}=\Phi_{\tau_{0}}(v)$, $u_{1}=\Phi_{\tau_{1}}(v)$ and $\tau_{0} \neq \tau_{1}$. Note that $\tau_{0}=\tau_{S_{B}}\left(u_{0}\right)$ and $\tau_{1}=\tau_{S_{B}}\left(u_{1}\right)$. Since $B$ is a box of length $l_{B}$, we can find for $i=0,1$ real numbers $\epsilon_{i}>0$ such that $\Phi_{\left[-l_{B} / 2, l_{B} / 2\right]}\left(u_{i}\right) \subseteq B$ and $\Phi_{\tau}\left(u_{i}\right) \notin B$ for $\tau \in\left(-l_{B} / 2-\epsilon_{i},-l_{B} / 2\right) \cup\left(l_{B} / 2, l_{B} / 2+\epsilon_{i}\right)$. This implies $\left|\tau_{1}-\tau_{0}\right|>l_{B}$. But by the definition of $U,\left|\tau_{1}-\tau_{0}\right|=\left|\tau_{S_{B}}\left(u_{1}\right)-\tau_{S_{B}}\left(u_{0}\right)\right| \leq$ $\left|\tau_{S_{B}}\left(u_{1}\right)-\tau_{S_{B}}(x)\right|+\left|\tau_{S_{B}}(x)-\tau_{S_{B}}\left(u_{0}\right)\right| \leq l_{B}$. This is the required contradiction.

Next we show for $x \in X$ that the existence of nonequivariant box containing $x$ in its interior already implies the existence of an equivariant box containing $x$ in its interior. The basic idea of proof is an averaging process in the time direction of the flow applied to the central slice of a nonequivariant box. 
Lemma 2.14 Suppose for the point $x \in X$ that there is a nonequivariant box whose interior contains $x$.

Then there exists a box $B$ in the sense of Definition 2.3 satisfying

(i) $G_{B}=G_{S_{B}}=G_{x}$;

(ii) $x \in S_{B} \cap B^{\circ}$;

(iii) $S_{B}$ is connected.

Proof Let $C$ be a nonequivariant box, ie a box in the sense of Definition 2.3 in the case, where the group $G$ is trivial, such that $x \in C^{\circ}$. Let $l=l_{C}$ be the length of $C$. Since the $G$-action on $X$ is proper by assumption and $X$ is metrizable, we can find a closed neighborhood $U$ of $x$ such that $U$ is a $\mathcal{F}$ in-subset of $X$ with $G_{U}=G_{x}$. We can assume without loss of generality that $x \in S_{C} \cap C^{\circ}$ and $C \subseteq U$ holds and for every $\tau \in[-l,-l / 2] \cup[l / 2, l]$ and $s \in S_{C}$ we have $\Phi_{\tau}(s) \notin C^{\circ}$, otherwise replace $C$ by an appropriate restriction. Let $S_{C}$ be the central slice of $C$. Let

$$
\mu: S_{C} \times[-l / 2, l / 2] \rightarrow C, \quad(s, \tau) \mapsto \Phi_{\tau}(s)
$$

be the homeomorphism of Lemma 2.6 (iii). Since $S_{C}$ is compact, $C^{\circ} \subseteq X$ is open, $G_{X}$ is finite and $X$ is metrizable, we can find a compact neighborhood $S_{0} \subseteq S_{C} \cap C^{\circ}$ of $x \in S_{C} \cap C^{\circ}$ such that $g S_{0} \subseteq C^{\circ}$ holds for all $g \in G_{x}$. Define

$$
S_{1}=\bigcap_{g \in G_{x}} S_{0} \cap \pi_{C}\left(\mu^{-1}\left(g S_{0}\right)\right),
$$

where $\pi_{C}: S_{C} \times[-l / 2, l / 2] \rightarrow S_{C}$ is the projection. Then $S_{1} \subseteq S_{0}$ is a compact neighborhood of $x$ in $S_{0}$. By construction there exists for every $g \in G_{x}$ and $s \in S_{1}$ precisely one element $\tau_{g}(s) \in(-l / 2, l / 2)$ such that $\Phi_{\tau_{g}(s)}(s) \in g S_{0}$. The function $\tau_{g}(s)$ is continuous in $s$ and has image in $(-l / 2+\delta, l / 2-\delta)$ for some $\delta$ with $0<\delta<l / 2$, since it is the restriction to $S_{1}$ of the continuous function with a compact source

$$
\tau_{g}: S_{0} \rightarrow(-l / 2, l / 2), \quad s \mapsto-\pi_{C} \circ \mu^{-1}\left(g^{-1} s\right) .
$$

Define the continuous function

$$
\tau: S_{1} \rightarrow(-l / 2, l / 2), \quad s \mapsto \frac{1}{\left|G_{x}\right|} \cdot \sum_{g \in G_{x}} \tau_{g}(s) .
$$

Put

$$
S_{2}=\left\{\Phi_{\tau(s)}(s) \mid s \in S_{1}\right\}
$$


Next we show that $S_{2} \subseteq C$ is $G_{x}$-invariant. Consider $g^{\prime} \in G_{x}$ and $u \in S_{2}$. Write $u=\Phi_{\tau(s)}(s)$ for appropriate $s \in S_{1}$. Let $s^{\prime} \in S_{0}$ be the element uniquely determined by $\Phi_{\tau_{\left(g^{\prime}\right)-1}(s)}(s)=\left(g^{\prime}\right)^{-1} s^{\prime}$. Then we get for $g \in G_{x}$

$\Phi_{\tau_{g}(s)-\tau_{\left(g^{\prime}\right)^{-1}}(s)}\left(s^{\prime}\right)=\Phi_{\tau_{g}(s)} \circ \Phi_{-\tau_{\left(g^{\prime}\right)^{-1}}(s)}\left(s^{\prime}\right)=\Phi_{\tau_{g}(s)}\left(g^{\prime} s\right)=g^{\prime} \Phi_{\tau_{g}(s)}(s) \in g^{\prime} g S_{0}$.

Since $g^{\prime} g S_{0} \subseteq C^{\circ}$ and $\tau_{g}(s)$ and $\tau_{\left(g^{\prime}\right)^{-1}}(s)$ belong to $(-l / 2, l / 2)$ and hence $\tau_{g}(s)-$ $\tau_{\left(g^{\prime}\right)^{-1}}(s) \in(-l, l)$ we conclude $\tau_{g}(s)-\tau_{\left(g^{\prime}\right)^{-1}}(s) \in(-l / 2, l / 2)$. Hence $s^{\prime} \in S_{1}$ and $\tau_{g^{\prime} g}\left(s^{\prime}\right)=\tau_{g}(s)-\tau_{\left(g^{\prime}\right)^{-1}}(s)$. Since this implies $\tau\left(s^{\prime}\right)=\tau(s)-\tau_{\left(g^{\prime}\right)^{-1}}(s)$, we conclude

$$
\begin{aligned}
g^{\prime} \cdot u & =g^{\prime} \cdot \Phi_{\tau(s)}(s)=\Phi_{\tau(s)}\left(g^{\prime} s\right)=\Phi_{\tau\left(s^{\prime}\right)+\tau_{\left(g^{\prime}\right)-1}(s)}\left(g^{\prime} s\right) \\
& =\Phi_{\tau\left(s^{\prime}\right)}\left(g^{\prime} \cdot \Phi_{\tau_{\left(g^{\prime}\right)-1}(s)}(s)\right)=\Phi_{\tau\left(s^{\prime}\right)}\left(g^{\prime} \cdot\left(g^{\prime}\right)^{-1} s^{\prime}\right)=\Phi_{\tau\left(s^{\prime}\right)} s^{\prime} \in S_{2} .
\end{aligned}
$$

Since $S_{1} \subseteq S_{C} \cap C^{\circ}$ is compact, $S_{2}$ is a compact $G_{x}$-invariant subset of $C^{\circ}$ with $x \in S_{2}$. Let $S_{3}$ be the component of $S_{2}$ which contains $x$. Then $S_{3}$ is a connected closed subset of $S_{2}$. Since $g S_{3} \cap S_{3}$ contains $x$ for $g \in G_{x}$, the subset $S_{3}$ is $G_{x}-$ invariant. Thus $S_{3} \subseteq C^{\circ}$ is a compact connected $G_{x}$-invariant subspace containing $x$.

We can find $\delta$ with $0<\delta<l / 2$ such that $B \subseteq C^{\circ}$ holds for $B:=\Phi_{[-\delta / 2, \delta / 2]}\left(S_{3}\right)$.

Next we show that $B$ is a box of length $\delta$. Since $S_{3}$ is $G_{x}$-invariant and the flow $\Phi$ commutes with the $G$-action the subset $B \subseteq X$ is $G_{X}$-invariant. Recall that $B \subseteq C$ holds and $C$ is a $\mathcal{F}$ in-subset of $X$ with $G_{C}=G_{X}$. Hence $B$ is a compact $\mathcal{F}$ in-subset of $X$. Consider $y \in B$. There is precisely one element $s \in S_{3}$ and $\tau \in[-\delta / 2, \delta / 2]$ satisfying $y=\Phi_{\tau}(s)$ since $S_{C}$ and hence $S_{3}$ is transversal to the flow with respect to $C$. Put $a_{-}(y)=-\delta / 2-\tau, a_{+}(y)=\delta / 2-\tau, \epsilon_{-}(y)=\epsilon_{+}(y)=l / 2$. Then

$$
\begin{aligned}
\delta & =a_{+}(y)-a_{-}(y) ; \\
\Phi_{\tau}(y) & \in B \text { for } \tau \in\left[a_{-}(y), a_{+}(y)\right] ; \\
\Phi_{\tau}(y) & \notin B \text { for } \tau \in\left(a_{-}(y)-\epsilon(y), a_{-}(y)\right) \cup\left(a_{+}(y), a_{+}(y)+\epsilon(y)\right) .
\end{aligned}
$$

Hence $B$ is a box with connected central slice $S_{B}=S_{3}$. We have $x \in S_{3}$. The projection $\pi_{C}: C \rightarrow S_{C}$ induces a homeomorphism $S_{2} \rightarrow S_{1}$ and maps the component $S_{3}$ of $S_{2}$ to a component $S_{1}^{\prime}$ of $S_{1}$. Since $S_{1}$ is an open neighborhood of $x$ in the space $S_{C} \cap C^{\circ}$ which is locally connected by Lemma 2.6 (iv), the component $S_{1}^{\prime}$ is a neighborhood of $x$ in the space $S_{C} \cap C^{\circ}$. Since $\tau$ is continuous we conclude from Lemma 2.6 (iii) that $x$ lies in the interior of $B$.

Definition 2.15 For $x \in X$ define its $G$-period

$$
\operatorname{per}_{\Phi}^{G}(x)=\inf \left\{\tau \mid \tau>0, \exists g \in G \text { with } \Phi_{\tau}(x)=g x\right\} \quad \in[0, \infty],
$$


where the infimum over the empty set is defined to be $\infty$. If $L \subseteq X$ is an orbit of the flow $\Phi$, define its $G$-period by

$$
\operatorname{per}_{\Phi}^{G}(L):=\operatorname{per}_{\Phi}^{G}(x)
$$

for any choice of $x \in X$ with $L=\Phi_{\mathbb{R}}(x)$.

For $r \geq 0$ put

$$
\begin{aligned}
& X_{>r}:=\left\{x \in X \mid \operatorname{per}_{\Phi}^{G}(x)>r\right\} ; \\
& X_{\leq r}:=\left\{x \in X \mid \operatorname{per}_{\Phi}^{G}(x) \leq r\right\} .
\end{aligned}
$$

Consider $x \in X$. Then the $G$-period $\operatorname{per}_{\Phi}^{G}(x)$ vanishes if and only if $x \in X^{\mathbb{R}}$. We have $\operatorname{per}_{\Phi}^{G}(x)=\infty$ if and only if the orbit through $x$ is not periodic and $g \Phi_{\mathbb{R}}(x) \cap \Phi_{\mathbb{R}}(x)=$ $\varnothing$ holds for all $g \neq 1$, or, equivalently, the orbit through $G x$ in the quotient space $G \backslash X$ with respect to the induced flow is not periodic. If $0<\operatorname{per}_{\Phi}^{G}(x)<\infty$, then the properness of the $G$-action implies the existence of $g \in G$ such that $\Phi_{\operatorname{per}_{\Phi}^{G}(x)}(x)=g x$ and $\operatorname{per}_{\Phi}^{G}(x)$ is the period of the periodic orbit through $G x$ in the quotient space $G \backslash X$ with respect to the induced flow.

Next we show for a point $x$ that we can find an equivariant box around a given compact part of the flow line, where the compact part is as long as the $G$-orbit length allows. The idea of proof is to take an equivariant box which contains $x$ in its interior, making its central slice very small by restriction and then prolonging the box along the flow line though $x$.

Lemma 2.16 Suppose for the point $x \in X$ that there is a nonequivariant box whose interior contains $x$. Consider a real number $l$ with $0<l<\operatorname{per}_{\Phi}^{G}(x)$.

Then we can find a box $C$ which satisfies

- $l_{C}=l$;

- $G_{C}=G_{x}$

- $x \in S_{C} \cap C^{\circ}$;

- $S_{C}$ is connected.

Proof From Lemma 2.14 we conclude the existence of a box $B$ in the sense of Definition 2.3 which satisfies $G_{B}=G_{x}, S_{B}$ is connected and $x \in S_{B} \cap B^{\circ}$. Let $l_{B}$ be the length of $B$. From Lemma 2.6 (v) we obtain a number $\epsilon_{B}>0$ such that for every $y \in S_{B}$ and $\tau \in\left(-l_{B} / 2-\epsilon_{B},-l_{B} / 2\right) \cup\left(l_{B} / 2, l_{B} / 2+\epsilon_{B}\right)$ the element $\Phi_{\tau}(y)$ does not belong to $B$. We can arrange by restricting $B$ and diminishing $\epsilon_{B}$ that $l_{B}<l$ and $l+\epsilon_{B}<\operatorname{per}_{\Phi}^{G}(x)$ holds. 
Next we show

$$
\Phi_{[-l / 2, l / 2]}(x) \cap g \Phi_{[-l / 2, l / 2]}(x) \neq \varnothing \Rightarrow g \in G_{x} .
$$

Namely, consider $y \in \Phi_{[-l / 2, l / 2]}(x) \cap g \Phi_{[-l / 2, l / 2]}(x)$. Then $y=\Phi_{\tau}(x)=g \Phi_{\sigma}(x)$ for appropriate $\tau, \sigma \in[-l / 2, l / 2]$. This implies $\Phi_{\tau-\sigma}(x)=g x$ and $|\tau-\sigma| \leq l<\operatorname{per}_{\Phi}^{G}(x)$. We conclude $\tau-\sigma=0$ and hence $g \in G_{X}$.

Since the $G$-action on $X$ is proper, we can find a closed neighborhood $V_{x}^{1} \subseteq X$ of $x$ such that $\Phi_{[-l / 2, l / 2]}\left(V_{x}^{1}\right) \cap g \Phi_{[-l / 2, l / 2]}\left(V_{x}^{1}\right) \neq \varnothing \Rightarrow g \in G_{x}$ holds. From $l+\epsilon_{B}<\operatorname{per}_{\Phi}^{G}(x)$ we conclude that

$$
\begin{array}{r}
\Phi_{\left[-l / 2+l_{B}, l / 2\right]}(x) \cap \Phi_{\left[-l / 2-\epsilon_{B},-l / 2\right]}(x)=\varnothing ; \\
\Phi_{\left[-l / 2, l / 2-l_{B}\right]}(x) \cap \Phi_{\left[l / 2, l / 2+\epsilon_{B}\right]}(x)=\varnothing .
\end{array}
$$

Since $\Phi$ is continuous and $\left[-l / 2+l_{B}, l / 2\right],\left[-l / 2-\epsilon_{B},-l / 2\right],\left[-l / 2, l / 2-l_{B}\right]$ and $\left[l / 2, l / 2+\epsilon_{B}\right]$ are compact, we can find a closed neighborhood $V_{x}^{2} \subseteq X$ of $x$ such that

$$
\begin{gathered}
\Phi_{\left[-l / 2+l_{B}, l / 2\right]}\left(V_{x}^{2}\right) \cap \Phi_{\left[-l / 2-\epsilon_{B},-l / 2\right]}\left(V_{x}^{2}\right)=\varnothing ; \\
\Phi_{\left[-l / 2, l / 2-l_{B}\right]}\left(V_{x}^{2}\right) \cap \Phi_{\left[l / 2, l / 2+\epsilon_{B}\right]}\left(V_{x}^{2}\right)=\varnothing .
\end{gathered}
$$

Put

$$
V_{x}=\bigcap_{g \in G_{x}} g \cdot\left(V_{x}^{1} \cap V_{x}^{2}\right) .
$$

Then $V_{x} \subseteq X$ is a closed $G_{x}$-invariant neighborhood of $x$ with the properties

- $\Phi_{[-l / 2, l / 2]}\left(V_{x}\right)$ is a $\mathcal{F}$ in-subset of the $G$-space $X$;

- $G_{\Phi_{[-l / 2, l / 2]}\left(V_{x}\right)}=G_{x}$;

- $\Phi_{\left[-l / 2+l_{B}, l / 2\right]}\left(V_{x}\right) \cap \Phi_{\left[-l / 2-\epsilon_{B},-l / 2\right]}\left(V_{x}\right)=\varnothing$;

- $\Phi_{\left[-l / 2, l / 2-l_{B}\right]}\left(V_{x}\right) \cap \Phi_{\left[l / 2, l / 2+\epsilon_{B}\right]}\left(V_{x}\right)=\varnothing$.

Let $V_{x}^{\circ} \subseteq X-X^{\mathbb{R}}$ be the interior of $V_{x}$. Let $T$ be the component of $S_{B} \cap B^{\circ} \cap V_{x}^{\circ}$ that contains $x$. Since $S_{B} \cap B^{\circ}$ is locally connected by Lemma 2.6 (iv) and $S_{B} \cap B^{\circ} \cap V_{x}^{\circ}$ is an open subset of $S_{B} \cap B^{\circ}$, the component $T$ is an open subset of $S_{B} \cap B^{\circ} \cap V_{x}^{\circ}$ and hence of $S_{B}$. Let $\bar{T}$ be the closure of $T$ in $S_{B}$. This is a closed connected $G_{X}$-invariant neighborhood of $x \in S_{B}$ which is contained in $V_{x}$. Since $S_{B}$ is a $\mathcal{F}$ in-subset of $X$ with $G_{S_{B}}=G_{X}, \bar{T}$ is a $\mathcal{F}$ in-subset of $S_{B}$ and we can consider the restriction $B(\bar{T})$. We can assume without loss of generality that the central slice $S_{B}$ is a $G_{X}$-invariant connected subset of $V_{x}$, otherwise replace $B$ by the restriction $B(\bar{T})$.

We define $C:=\Phi_{[-l / 2, l / 2]}\left(S_{B}\right)$. 
Next we show that $C$ is a box of length $l$. Since $C \subseteq \Phi_{[-l / 2, l / 2]}\left(V_{x}\right)$ and $C$ is $G_{x}$-invariant, $C$ is a compact $\mathcal{F}$ in-subset of the $G$-space $X$. Consider $y \in C$. We can write it as $y=\Phi_{\tau_{y}}(s)$ for $\tau_{y} \in[-l / 2, l / 2]$ and $s \in S_{B}$. Put $a_{-}(y)=$ $-l / 2-\tau_{y}$ and $a_{+}(y)=l / 2-\tau_{y}$. Obviously $l=a_{+}(y)-a_{-}(y)$ and $\Phi_{\tau}(y) \in C$ for $\tau \in\left[a_{-}(y), a_{+}(y)\right]$. It remains to show that $\Phi_{\tau^{\prime}}(y) \notin C$ holds for $\tau^{\prime} \in\left(a_{-}(y)-\right.$ $\left.\epsilon_{B}, a_{-}(y)\right) \cup\left(a_{+}(y), a_{+}(y)+\epsilon_{B}\right)$. This is equivalent to showing that $\Phi_{\tau^{\prime}}(s) \notin C$ holds for $\tau^{\prime} \in\left(-l / 2-\epsilon_{B},-l / 2\right) \cup\left(l / 2, l / 2+\epsilon_{B}\right)$. Since $s \in S_{B} \subseteq V_{x}$, we have

$$
\begin{gathered}
\Phi_{\left[-l / 2+l_{B}, l / 2\right]}\left(S_{B}\right) \cap \Phi_{\left[-l / 2-\epsilon_{B},-l / 2\right]}(s)=\varnothing ; \\
\Phi_{\left[-l / 2, l / 2-l_{B}\right]}\left(S_{B}\right) \cap \Phi_{\left[l / 2, l / 2+\epsilon_{B}\right]}(s)=\varnothing .
\end{gathered}
$$

The main property of $\epsilon_{B}$ is

$$
\Phi_{\left[-l_{B} / 2, l_{B} / 2\right]}\left(S_{B}\right) \cap \Phi_{\left(-l_{B} / 2-\epsilon_{B},-l_{B} / 2\right) \cup\left(l_{B} / 2, l_{B} / 2+\epsilon_{B}\right)}(s)=\varnothing .
$$

Applying $\Phi_{\left(l_{B}-l\right) / 2}$ respectively $\Phi_{\left(l-l_{B}\right) / 2}$ we obtain

$$
\begin{array}{r}
\Phi_{\left[-l / 2,-l / 2+l_{B}\right]}\left(S_{B}\right) \cap \Phi_{\left(-l / 2-\epsilon_{B},-l / 2\right)}(s)=\varnothing ; \\
\Phi_{\left[l / 2-l_{B}, l / 2\right]}\left(S_{B}\right) \cap \Phi_{\left(l / 2, l / 2+\epsilon_{B}\right)}(s)=\varnothing .
\end{array}
$$

We conclude

$$
\Phi_{[-l / 2, l / 2]}\left(S_{B}\right) \cap \Phi_{\left(-l / 2-\epsilon_{B},-l / 2\right) \cup\left(l / 2, l / 2+\epsilon_{B}\right)}(s)=\varnothing .
$$

Hence $C$ is a box of length $l$. By construction $S_{C}=S_{B}$ is connected, $G_{C}=G_{S_{C}}=G_{x}$ and $x \in S_{B} \cap B^{\circ}$ and hence $x \in S_{C} \cap C^{\circ}$.

Lemma 2.17 Consider real numbers $a, b, c>0$ satisfying $c>a+2 b$. Let $K$ be a cocompact $G$-invariant subset of $X_{>a+2 b+2 c}$.

Then there exist a $G$-set $\Lambda$ and for every $\lambda \in \Lambda$ boxes $A_{\lambda} \subseteq B_{\lambda} \subseteq C_{\lambda}$ such that:

(i) $\Lambda$ is $G$-cofinite;

(ii) We have

$$
\begin{aligned}
& l_{A_{\lambda}}=a ; \\
& l_{B_{\lambda}}=a+2 b ; \\
& l_{C_{\lambda}}=a+2 b+2 c ;
\end{aligned}
$$

(iii) $S_{C_{\lambda}}$ is connected;

(iv) We have $S_{A_{\lambda}} \subseteq S_{B_{\lambda}} \subseteq S_{C_{\lambda}}$;

(v) $A_{\lambda} \subseteq B_{\lambda}^{\circ}$ and $B_{\lambda} \subseteq C_{\lambda}^{\circ}$;

(vi) $K \subseteq \bigcup_{\lambda \in \Lambda} A_{\lambda}^{\circ}$; 
(vii) $g A_{\lambda}=A_{g \lambda}, g B_{\lambda}=B_{g \lambda}$ and $g C_{\lambda}=C_{g \lambda}$ for $g \in G$;

(viii) If $B_{\lambda} \cap B_{\lambda^{\prime}} \neq \varnothing$, then $B_{\lambda} \subseteq C_{\lambda^{\prime}}^{\circ}$ and $S_{B_{\lambda}}$ is transversal to the flow with respect to $C_{\lambda^{\prime}}$.

Proof Lemma 2.16 implies that we can find for every $x \in X_{>a+2 b+2 c}$ a box $C_{x}$ of length $a+2 b+2 c$ such that $x \in S_{C_{x}} \cap C_{x}^{\circ}$ and $G_{C_{x}}=G_{x}$ holds and $S_{C_{x}}$ is connected. Since $G_{x}$ is finite, we can find a $G_{x}$-invariant closed neighborhood $T_{x}$ of $x$ in $S_{C_{x}}$ such that $T_{x} \subseteq S_{C_{x}} \cap C_{x}^{\circ}$ holds. We can arrange that $g C_{x}=C_{g x}$ and $g T_{x}=T_{g x}$ holds for $g \in G$ and $x \in X_{>a+2 b+2 c}$. Obviously $K \subseteq \bigcup_{x \in K} C_{x}\left(T_{x} ; a\right)^{\circ}$, where we use the notation from Definition 2.10. Since $K$ is cocompact and $G$-invariant, we can find a cofinite $G$-subset $I \subseteq K$ satisfying

$$
K \subseteq \bigcup_{x \in I} C_{x}\left(T_{x} ; a\right)^{\circ}
$$

Fix $x \in I$. Consider $y \in T_{x}$. Since the $G$-action is proper, $g \cdot C_{z}\left(T_{z}\right)=C_{g z}\left(T_{g z}\right)$ holds for $z \in I$ and $g \in G$ and $C_{x}(\{y\} ; a+2 b)$ and $C_{z}\left(T_{z} ; a+2 b\right)$ are compact, we can find a closed $G_{y}$-invariant neighborhood $V_{y} \subseteq T_{x}$ of $y$ such that for all $z \in I$

$$
C_{x}(\{y\} ; a+2 b) \cap C_{z}\left(T_{z} ; a+2 b\right)=\varnothing \Rightarrow C_{x}\left(V_{y} ; a+2 b\right) \cap C_{z}\left(T_{z} ; a+2 b\right)=\varnothing .
$$

For $y \in T_{x}$ we define

$$
I_{y}=\left\{z \in I \mid C_{x}(\{y\} ; a+2 b) \cap C_{z}^{\circ} \neq \varnothing\right\} .
$$

Since the set $G$-set $I$ is cofinite, $C_{x}(\{y\} ; a+2 b)$ and $C_{z}$ are compact and the $G$-action on $X$ is proper, the set $I_{y}$ is finite. From $a+2 b<c$ and $l_{C_{z}}=a+2 b+2 c$ we conclude for $z \in I_{y}$ that $C_{x}(\{y\} ; a+2 b)=\Phi_{[-a / 2-b, a / 2+b]}(y)$ is contained in $C_{z}^{\circ}$. Since $C_{x}(\{y\} ; a+2 b)$ is compact, we can find for $z \in I_{y}$ a closed $G_{y}$-invariant neighborhood $U_{y}(z) \subseteq T_{x}$ of $y$ such that $C_{x}\left(U_{y}(z) ; a+2 b\right)=\Phi_{[-a / 2-b, a / 2+b]}\left(U_{y}(z)\right)$ is contained in $C_{z}^{\circ}$. Because of Lemma 2.13 applied to $C_{x}\left(U_{y}(z) ; a+2 b\right) \subseteq C_{z}$ we can assume without loss of generality that $U_{y}(z)$ is transversal to the flow with respect to $C_{z}$ for every $z \in I_{y}$.

Put $U_{y}:=V_{y} \cap \bigcap_{z \in I_{y}} U_{y}(z)$. Then $U_{y} \subseteq T_{x}$ is a $G_{y}$-invariant closed neighborhood of $y$ such that

$$
C_{x}\left(U_{y} ; a+2 b\right) \subseteq C_{z}^{\circ} \quad \text { if } z \in I_{y} ;
$$

$$
U_{y} \text { is transversal to the flow with respect to } C_{z} \text { for } z \in I_{y} \text {; }
$$

$$
\begin{aligned}
& C_{x}\left(U_{y} ; a+2 b\right) \cap C_{z}\left(T_{z} ; a+2 b\right)=\varnothing \\
& \text { if } z \in I \text { and } C_{x}(\{y\} ; a+2 b) \cap C_{z}\left(T_{z} ; a+2 b\right)=\varnothing .
\end{aligned}
$$


Choose a $G_{y}$-invariant closed neighborhood $W_{y} \subseteq T_{x}$ of $y$ such that $W_{y} \subseteq U_{y}^{\circ}$. Obviously $T_{x}=\bigcup_{y \in T_{x}} W_{y}^{\circ}$. Since $T_{x}$ is compact, we can find $y(x)_{1}, y(x)_{2}, \ldots, y(x)_{n(x)}$ in $T_{x}$ such that $T_{x}=\bigcup_{i=1}^{n(x)} W_{y(x)_{i}}$. We can arrange $W_{g y}=g W_{y}, n(x)=n(g x)$ and $y(g x)_{i}=g y(x)_{i}$ for $g \in G$ since $C_{x}$ is a $\mathcal{F}$ in-subset of $X$ with $G_{C_{x}}=G_{x}$. Obviously

$$
C_{x}\left(T_{x} ; a\right)^{\circ}=\bigcup_{i=1}^{n(x)} C_{x}\left(W_{y(x)_{i}} ; a\right)^{\circ} .
$$

Define

$$
\Lambda=\left\{y_{i}(x) \mid x \in I, i \in\{1,2, \ldots, n(x)\}\right\} .
$$

This is a cofinite $G$-set. Define for $\lambda=y_{i}(x)$ in $\Lambda$

$$
\begin{aligned}
& C_{\lambda}=C_{x} ; \\
& B_{\lambda}=C_{x}\left(U_{y_{i}(x)} ; a+2 b\right) ; \\
& A_{\lambda}=C_{x}\left(W_{y_{i}(x)} ; a\right) .
\end{aligned}
$$

It remains to check that this collection of boxes has the desired properties. This is obvious for assertions (i), (ii), (iii), (iv) and (vii). Assertion (v) follows from $W_{y} \subseteq U_{y}^{\circ}$ and $U_{y} \subseteq S_{C_{x}} \cap C_{x}^{\circ}$. Assertion (vi) follows from (2.18) and (2.22).

Finally we prove assertion (viii). Suppose that $B_{\lambda} \cap B_{\lambda^{\prime}} \neq \varnothing$. Write $\lambda=y_{i}(x)$ and $\lambda^{\prime}=y_{i^{\prime}}\left(x^{\prime}\right)$. Since $B_{\lambda}=C_{x}\left(U_{y_{i}(x)} ; a+2 b\right)$ and $B_{\lambda^{\prime}} \subseteq C_{x^{\prime}}\left(T_{x^{\prime}} ; a+2 b\right)$, we conclude that $C_{x}\left(U_{y_{i}(x)} ; a+2 b\right) \cap C_{x^{\prime}}\left(T_{x^{\prime}} ; a+2 b\right) \neq \varnothing$. By (2.21) we have $x^{\prime} \in I_{y_{i}(x)}$. We conclude from (2.19)

$$
B_{\lambda}=C_{x}\left(U_{y_{i}(x)} ; a+2 b\right) \subseteq C_{x^{\prime}}^{\circ}=C_{\lambda^{\prime}}^{\circ} .
$$

The central slice $S_{B_{\lambda}}=U_{y_{i}(x)}$ is transversal to the flow with respect to $C_{\lambda^{\prime}}$ by (2.20). This finishes the proof of Lemma 2.17.

\section{General position in metric spaces}

Definition 3.1 Let $Z$ be a metric space, $A \subseteq Z$ and $\delta>0$. Then we define the sets

$$
\begin{aligned}
A^{\delta} & =\{x \in Z \mid \exists a \in A \text { such that } d(x, a)<\delta\}, \\
A^{-\delta} & =\{x \in A \mid d(x, Z-A)>\delta\} .
\end{aligned}
$$

For $x \in Z$, we will abbreviate $x^{\delta}=\{x\}^{\delta}$.

Notice that $A^{\delta}$ and $A^{-\delta}$ are open. The following Propositions 3.2 and 3.3 are the main results of this section which is entirely devoted to their proof. 
Proposition 3.2 Let $Z$ be a compact metrizable space of covering dimension $n$ with an action of a finite group $F$. Let $\mathcal{U}$ be a finite collection of open $\mathcal{F}$ in-subsets such that $g U \in \mathcal{U}$ for $g \in F, U \in \mathcal{U}$. Suppose that we are given for each $U \in \mathcal{U}$ an open subset $U^{\prime \prime} \subseteq U$ satisfying $\overline{U^{\prime \prime}} \subseteq U$. Put $m=(n+1) \cdot|F|$.

Then for each $U \in \mathcal{U}$ we can find an open subset $U^{\prime} \subseteq Z$ such that:

(i) $U^{\prime \prime} \subseteq \overline{U^{\prime \prime}} \subseteq U^{\prime} \subseteq \overline{U^{\prime}} \subseteq U$;

(ii) If $\mathcal{U}_{0} \subseteq \mathcal{U}$ has more than $m$ elements, then

$$
\bigcap_{U \in \mathcal{U}_{0}} \partial U^{\prime}=\varnothing
$$

(iii) $(g U)^{\prime}=g\left(U^{\prime}\right)$ for $g \in F, U \in \mathcal{U}$.

Proposition 3.3 Let $Z$ be a compact metric space of covering dimension $n$ with an action of a finite group $F$ by isometries. Let $Y \subseteq Z$ be an open locally connected $F$-invariant subset. Let $\mathcal{U}$ be a finite collection of open subsets such that $g U \in \mathcal{U}$ for $g \in F, U \in \mathcal{U}$ and $\bar{U} \subseteq Y$ for $U \in \mathcal{U}$ holds. Assume that there is $k$ such that for every subset $\mathcal{U}_{0} \subseteq \mathcal{U}$ with more than $k$ elements we have

$$
\bigcap_{U \in \mathcal{U}_{0}} \partial U=\varnothing
$$

Let $\delta>0$. Put $m=(n+1) \cdot|F|, l=k|F|$.

Then there are finite collections $\mathcal{V}^{j}, j=0, \ldots, m$ of open subsets of $Z$, such that:

(i) $\mathcal{V}=\mathcal{V}^{0} \cup \cdots \cup \mathcal{V}^{m}$ is an open cover of $Z$;

(ii) $\operatorname{diam}(V)<\delta$ for every $V \in \mathcal{V}$;

(iii) For $V \in \mathcal{V}$ there are at most $l$ different sets $U \in \mathcal{U}$ such that $U$ and $V$ intersect, but $U$ does not contain $V$;

(iv) For fixed $j$ and $V_{0} \in \mathcal{V}^{j}$ we have $V_{0} \cap V \neq 0$ for at most $2^{j+1}-2$ different sets $V \in \mathcal{V}^{0} \cup \cdots \cup \mathcal{V}^{j}$;

(v) For fixed $j$ and $V_{0}, V_{1} \in \mathcal{V}^{j}$ we have either $V_{0}=V_{1}$ or $\overline{V_{0}} \cap \overline{V_{1}}=\varnothing$;

(vi) Each $\mathcal{V}^{i}$ is $F$-invariant, ie, $g V \in \mathcal{V}^{i}$ for $g \in F, V \in \mathcal{V}^{i}$;

(vii) $V=\bar{V}^{\circ}$ for $V \in \mathcal{V}$.

In order to prove these two propositions we will compare the metric space $Z$ to the nerve of a suitable open cover of $Z$. The results will be first proven for simplicial complexes and then be pulled back to $Z$ using the map from the next remark. 
Remark 3.4 Let $Z$ be a metric space and $\mathcal{U}$ be a locally finite open cover of $Z$. Denote by $\mathcal{N}(\mathcal{U})$ the simplicial complex given by the nerve of $\mathcal{U}$. Then

$$
z \mapsto \sum_{U \in \mathcal{U}}\left(\frac{d(z, Z-U)}{\sum_{V \in \mathcal{U}} d(z, Z-V)}\right)[U]
$$

defines a map $f_{\mathcal{U}}: Z \rightarrow \mathcal{N}(\mathcal{U})$, where $[U]$ denotes the vertex of $\mathcal{N}(\mathcal{U})$ that corresponds to $U \in \mathcal{U}$. If a group $G$ acts by isometries on $Z$ and on the cover $\mathcal{U}$ (ie, $g U \in \mathcal{U}$ for $g \in G, U \in \mathcal{U})$ then $f_{\mathcal{U}}$ is equivariant for the induced action on $\mathcal{N}(\mathcal{U})$.

Lemma 3.6 Let $f: Y \rightarrow Z$ be a continuous map between metric spaces. Assume that $Y$ is compact. Let $U$ be an open subset of $Z$ and $\beta>0$. Then there exists $\alpha>0$ such that

$$
\left(f^{-1}(U)\right)^{-\beta} \subseteq f^{-1}\left(U^{-\alpha}\right) .
$$

Proof We proceed by contradiction and assume that for every $n \in \mathbb{N}$ there is $x_{n} \in Y$ such that

$$
\begin{aligned}
& x_{n} \in f^{-1}(U)^{-\beta}, \\
& x_{n} \notin f^{-1}\left(U^{-1 / n}\right) .
\end{aligned}
$$

By compactness of $Y$, we may assume $x_{n} \rightarrow x$ as $n \rightarrow \infty$. We derive from (3.8) that there is $z_{n} \in Z-U$ such that $d\left(z_{n}, f\left(x_{n}\right)\right) \leq 2 / n$. Then $z_{n} \rightarrow f(x)$ as $n \rightarrow \infty$ and thus $f(x) \notin U$. On the other hand (3.7) implies that $f\left(\left(x_{n}\right)^{\beta}\right) \subseteq U$. Since $x \in\left(x_{n}\right)^{\beta}$ for sufficiently large $n$ we have $f(x) \in U$. This is the desired contradiction.

In the sequel interior of a simplex means simplicial interior, ie, the simplex with all its proper faces removed.

Lemma 3.9 Let $Z$ be a simplicial complex and let $Z^{(n)}$ be the $n$-th barycentric subdivision of $Z, n \geq 1$. Let $\Delta$ be a simplex of $Z^{(n)}$. Let $\sigma, \tau$ be simplices of $Z$ such that both the interior of $\sigma$ and the interior of $\tau$ intersect $\Delta$. Then $\sigma \subseteq \tau$ or $\tau \subseteq \sigma$.

Proof Let $\Delta^{\prime}$ be a simplex of the first barycentric subdivision $Z^{\prime}=Z^{(1)}$ of $Z$ which contains $\Delta$. Then both the interior of $\sigma$ and the interior $\tau$ intersect $\Delta^{\prime}$. Thus it suffices to prove the claim for the first barycentric subdivision.

Now a $d$-simplex $\Delta^{\prime}$ of the first barycentric subdivision $Z^{\prime}$ of $Z$ is given by a sequence $\sigma_{0}, \sigma_{1}, \ldots, \sigma_{d}$ such that each $\sigma_{i}$ is a simplex of $Z$ and $\sigma_{i-1}$ is a proper face of $\sigma_{i}$ for $i=1,2, \ldots, d$. This is the simplex in the barycentric subdivision whose vertices are the barycenters of $\sigma_{0}, \sigma_{1}, \ldots, \sigma_{d}$. Then the simplices of $Z$ whose interior intersect $\Delta^{\prime}$ are precisely the $\sigma_{i}$. 
Recall that the open star of a vertex $e$ of a simplicial complex $Z$ is defined to be the set of all points $z \in Z$ whose carrier simplex has $v$ as an vertex. Equivalently, one obtains the star of $v$ by taking the union of all simplices $\sigma$ which have $v$ as vertex and then deleting those faces of these simplices $\sigma$ which do not have $v$ as a vertex. We will denote it by $\operatorname{star}(v)$. The set of open stars of vertices of $Z$ is an open covering of $Z$.

Let $\sigma$ be a simplex of $Z$. Define $\operatorname{star}^{\prime}(\sigma)$ to be the star in the first barycentric subdivision $Z^{\prime}$ of the vertex in $Z^{\prime}$ given by $\sigma$.

Proof of Proposition 3.3 Since $\mathcal{U}$ is finite and $\partial U$ closed for $U \in \mathcal{U}$, the assumptions on $\mathcal{U}$ imply that we can find for every $z \in Z$ an open neighborhood $W_{z}$ of $z$ such that for all $z \in Z$ the following holds.

- $\operatorname{diam}\left(W_{z}\right)<\delta /|F|$;

- $W_{z}$ intersects the boundary of at most $k$ sets in $\mathcal{U}$;

- If $W_{z}$ intersects $\bar{U}$ for some $U \in \mathcal{U}$, then $W_{z} \subseteq Y$.

Since the covering dimension of $Z$ is $n$ by assumption and $Z$ is compact, we can choose a finite open refinement $\mathcal{W}$ of the open cover $\left\{W_{z} \mid z \in Z\right\}$ such that $\operatorname{dim}(\mathcal{W}) \leq n$. Let $\mathcal{W}_{F}$ be the collection of subsets of $Z$ which consists of the components of subsets of the form $\bigcup_{g \in F} g W$ for $W \in \mathcal{W}$ with $W \subseteq Y$ and subsets of the form $g W$ for $g \in F$ and $W \in \mathcal{W}$ with $W \nsubseteq Y$. Since $Y$ is a locally connected open subset of $Z$, the above components are open subsets of $Z$. Thus the elements in the finite set $\mathcal{W}_{F}$ are open subsets. Hence $\mathcal{W}_{F}$ is a finite open covering of $Z$. Since $\operatorname{dim}(\mathcal{W}) \leq n$ every orbit of $F$ in $Z$ meets at most $(n+1) \cdot|F|$ members of $\mathcal{W}$. We conclude $\operatorname{dim}\left(\mathcal{W}_{F}\right) \leq m=(n+1) \cdot|F|$. Obviously $g V \in \mathcal{W}_{F}$ holds if $V \in \mathcal{W}_{F}$. If $V$ is a component of $\bigcup_{g \in F} g W$ for some $W \in \mathcal{W}$, we can find elements $g_{1}, g_{2}, \ldots, g_{r}$ in $F$ such that $V$ is contained in $\bigcup_{i=1}^{r} g_{i} W$ and $\left(\bigcup_{j=1}^{i} g_{j} W\right) \cap g_{i+1} W \neq \varnothing$ holds for $i=1,2, \ldots,(r-1)$. One shows by induction over $i=1,2 \ldots, r$ that the diameter of $\bigcup_{j=1}^{i} g_{j} W$ is less or equal to the sum of the diameters of the sets $g_{1} W, g_{2} W, \ldots$, $g_{i} \cdot W$. Hence the diameter of any element $V$ of $\mathcal{W}_{F}$ is bounded by $\delta$.

Consider $U \in \mathcal{U}$ and an element $V \in \mathcal{W}_{F}$ such that $V$ intersects $U$ but is not contained in $U$. By construction $V$ is a component of $\bigcup_{g \in F} g W$ for some $W \in \mathcal{W}$ with $W \subseteq Y$. Since $V$ is connected we must have $V \cap \partial U \neq \varnothing$. Hence there exists $g \in F$ with $g W \cap \partial U \neq \varnothing$, or, equivalently, with $W \cap g^{-1} \partial U \neq \varnothing$. Since each set $W_{z}$ intersects the boundary of at most $k$ sets in $\mathcal{U}$ and $g^{-1} U \in \mathcal{U}$, there are at most $l=|F| \cdot k$ elements $U \in \mathcal{U}$ satisfying $W \cap g^{-1} \partial U \neq \varnothing$ for some $g \in F$. Hence for every $V \in \mathcal{W}_{F}$ there are at most $l$ elements $U \in \mathcal{U}$ such that $V$ intersects $U$ but is not contained in $U$. 
Consider the map $f=f_{\mathcal{W}_{F}}: Z \rightarrow \mathcal{N}\left(\mathcal{W}_{F}\right)$ from Remark 3.4. Put for $j=0,1, \ldots, m$

$$
\mathcal{X}^{j}=\left\{\operatorname{star}^{\prime}(\sigma) \mid \sigma \in \mathcal{N}\left(\mathcal{W}_{F}\right), \operatorname{dim}(\sigma)=j\right\} .
$$

Then $\mathcal{X}=\mathcal{X}^{0} \cup \mathcal{X}^{1} \cup \ldots \cup \mathcal{X}^{m}$ is an open cover of $\mathcal{N}\left(\mathcal{W}_{F}\right)$.

Let $\sigma$ and $\tau$ be simplices in $Z$ with $\operatorname{star}^{\prime}(\sigma) \cap \operatorname{star}^{\prime}(\tau) \neq \varnothing$. Let $\Delta$ be the carrier simplex in $Z^{\prime}$ of some point in $\operatorname{star}^{\prime}(\sigma) \cap \operatorname{star}^{\prime}(\tau)$. Then the two vertices of $Z^{\prime}$ given by $\sigma$ and $\tau$ belong to $\Delta$. Hence the barycenters of $\sigma$ and $\tau$ belong to $\Delta$. In particular the interior of $\sigma$ and the interior of $\tau$ intersect $\Delta$. We conclude from Lemma 3.9 that $\sigma \subseteq \tau$ or $\tau \subseteq \sigma$ holds. This implies that the elements in $\mathcal{X}^{j}$ are pairwise disjoint and every $V \in \mathcal{X}^{j}$ intersects at most $2^{j+1}-2$ elements in $\left\{\mathcal{X}^{0} \cup \mathcal{X}^{1} \cup \ldots \cup \mathcal{X}^{j}\right\}$ since a $j$-simplex has $2^{j+1}-2$ proper faces.

If the simplex $\sigma$ in $\mathcal{N}\left(\mathcal{W}_{F}\right)$ is given by the subset $\left\{V_{0}, V_{1}, \ldots, V_{D}\right\} \subseteq \mathcal{W}_{F}$, then $f^{-1}\left(\operatorname{star}^{\prime}(\sigma)\right)$ is contained in $V_{0} \cap V_{1} \cap \ldots \cap V_{D}$, since $\operatorname{star}^{\prime}(\sigma)$ is contained in the intersection of the stars in $\mathcal{N}\left(\mathcal{W}_{F}\right)$ of the vertices of $\sigma$ and for $V \in \mathcal{N}\left(\mathcal{W}_{F}\right)$ we have $f^{-1}(\operatorname{star}(V)) \subseteq V$. Hence for every $X \in \mathcal{X}$ there exists $W \in \mathcal{W}_{F}$ with $f^{-1}(X) \subseteq W$. Put

$$
\hat{\mathcal{V}}^{j}=\left\{f_{\mathcal{W}_{F}}^{-1}(X) \mid X \in \mathcal{X}^{j}\right\}
$$

Then $\hat{\mathcal{V}}^{j}$ has the following properties:

- $\hat{\mathcal{V}}=\hat{\mathcal{V}}^{0} \cup \cdots \cup \hat{\mathcal{V}}^{m}$ is an open cover of $Z$ consisting of finitely many elements.

- $\operatorname{diam} \hat{V}<\delta$ for every $\widehat{V} \in \hat{\mathcal{V}}$;

- For $\hat{V} \in \hat{\mathcal{V}}$ there are at most $l$ different sets $U \in \mathcal{U}$ such that $U$ and $\hat{V}$ intersect, but $U$ does not contain $\hat{V}$;

- For fixed $j$, every $\widehat{V}_{0} \in \widehat{\mathcal{V}}^{j}$ intersects at most $2^{j+1}-2$ different sets $\hat{V} \in$ $\hat{\mathcal{V}}^{0} \cup \cdots \cup \widehat{\mathcal{V}}^{j}$

- For fixed $j$ and $\widehat{V}_{0}, \widehat{V}_{1} \in \hat{\mathcal{V}}^{j}$ we have either $\widehat{V}_{0}=\widehat{V}_{1}$ or $\widehat{V}_{0} \cap \widehat{V}_{1}=\varnothing$;

- Each $\hat{\mathcal{V}}^{i}$ is $F$-invariant, ie, $g \widehat{V} \in \hat{\mathcal{V}}^{i}$ for $g \in F, \widehat{V} \in \hat{\mathcal{V}}^{i}$.

For $\epsilon>0$ define

$$
\mathcal{V}^{j}=\left\{\left(\overline{(\widehat{V})^{-\epsilon}}\right)^{\circ} \mid \widehat{V} \in \hat{\mathcal{V}}^{j}\right\} .
$$

Then $\mathcal{V}^{j}$ has properties (ii), (iii), (iv), (v), (vi) and (vii) since $\left(\overline{(\widehat{V})^{-\epsilon}}\right)^{\circ} \subseteq \widehat{V}$ for $\hat{V} \in \mathcal{V}^{j^{\prime}}$ and we have $\bar{T}=\bar{T}^{\circ}$ for any open subset $T$ of a topological space. Since $Z$ is compact, we can choose $\epsilon$ so small that also property (i) is satisfied. This finishes the proof of Proposition 3.3. 
Proposition 3.10 Let $Z$ be a simplicial complex and let $Z^{(m)}$ be the $m$-th barycentric subdivision of $Z, m \geq 1$. Let $B$ be a subcomplex of $Z$. Let $B_{0}$ be the union of all simplices of $Z^{(m)}$ that are contained in the interior of $B$. Let $\beta$ be a simplex of $Z$ and let $\sigma$ be a simplex of $Z^{(m)}$ that is contained in the intersection of the boundary of $B_{0}$ with $\beta$. Then $\operatorname{dim}(\sigma)<\operatorname{dim}(\beta)$.

We recall that the topological interior and boundary of a subcomplex of a simplicial complex can be described combinatorial as follows: the interior is the union of all open simplices (simplices with all proper faces removed) that are contained in the subcomplex; its boundary is the union of all simplices that are contained in the subcomplex and are in a addition a face of simplex not contained in the subcomplex.

Proof of Proposition 3.10 By the definition of $B_{0}$ there exists a simplex $\Delta$ of $Z^{(m)}$ such that $\sigma \subseteq \Delta$, but $\Delta$ is not contained in the interior of $B$. Thus there exists a simplex $\alpha$ of $Z$ that is contained in the boundary of $B$ and intersects $\Delta$. By passing to a face of $\alpha$, we can arrange that $\alpha$ is contained in the boundary of $B$ and the interior of $\alpha$ intersects $\Delta$. Since $\sigma$ is contained in $\beta$, we can find a face $\beta^{\prime}$ of $\beta$ such that $\sigma$ intersects the interior of $\beta^{\prime}$. Hence the interior of the simplex $\beta^{\prime} \subseteq Z$ intersects $\Delta$. Lemma 3.9 implies $\alpha \subseteq \beta^{\prime}$ or $\beta^{\prime} \subseteq \alpha$. In the second case, $\sigma \subseteq \beta^{\prime} \subseteq \alpha$. But $\sigma$ has to be disjoint from $\alpha$, because $\alpha$ lies on the boundary of $B$, while $\sigma$ is contained in the interior of $B$. We conclude $\alpha \subseteq \beta^{\prime}$ and hence $\alpha \subseteq \beta$. Therefore, $\tau=\beta \cap \Delta$ is a simplex of $Z^{(m)}$, that contains the simplex $\sigma$ as a face and intersects $\alpha$. Since $\sigma$ and $\alpha$ are disjoint, $\sigma$ is a proper face of $\tau$. This implies $\operatorname{dim}(\sigma)<\operatorname{dim}(\tau) \leq \operatorname{dim}(\beta)$.

Proof of Proposition 3.2 The strategy is first to prove a simplicial version and then use the map appearing in Remark 3.4 to handle the general case of a metric space.

Since we assume that $Z$ is metrizable and $F$ is finite, we can choose a metric $d_{Z}$ on $Z$ which is $F$-invariant. Since $Z$ and hence each $\overline{U^{\prime \prime}}$ is compact and the collection $\mathcal{U}$ is finite, we can find $\delta>0$ such that $\overline{U^{\prime \prime}} \subseteq U^{-\delta}$ holds for $U \in \mathcal{U}$. Hence we can assume in the sequel without loss of generality that $U^{\prime \prime}=U^{-\delta}$.

So we start with the special case where $Z$ is in addition a simplicial complex, each $U \in \mathcal{U}$ is the interior of a subcomplex of $Z$ and $F$ acts simplicially on $Z$. Let $\left\{U_{1}, \ldots, U_{r}\right\} \subseteq \mathcal{U}$ contain exactly one element from every $F$-orbit in the $F$-set $\mathcal{U}$. Pick $m \geq 0$ such that the simplices of the $m$-th barycentric subdivision $Z^{(m)}$ of $Z$ have diameter $<\delta$. For $i=1,2, \ldots, r$ let $Z^{(m+i)}$ be the $(m+i)$-th barycentric subdivision of $Z$ and let $A_{i}$ be the union of all those simplices of $Z^{(m+i)}$ which are contained in $U_{i}$. Then $A_{i}$ is the largest subcomplex of $Z^{(m+i)}$ that is contained in $U_{i}$. Since each simplex of $Z^{(m+i)}$ has diameter $<\delta$, we get $U_{i}^{-\delta} \subseteq A_{i} \subseteq U_{i}$. 
Define $U_{i}^{\prime}$ to be the interior of $A_{i}$. For $U \in \mathcal{U}$ define $U^{\prime}=g U_{i}^{\prime}$ for any choice of $g \in F$ and $i \in\{1,2, \ldots, r\}$ satisfying $U=g U_{i}$. One easily checks that $i$ is uniquely determined by $U$ and the choice of $g \in F$ does not matter in the definition of $U^{\prime}$ since each $U$ is by assumption a $\mathcal{F}$ in-subset. Obviously (i) and (iii) are satisfied for $\mathcal{U}^{\prime}=\left\{U^{\prime} \mid U \in \mathcal{U}\right\}$.

Next we prove (ii) but with $m$ replaced by $n=\operatorname{dim}(Z)$. Consider a subset $\mathcal{U}_{0} \subseteq \mathcal{U}$ with $\left|\mathcal{U}_{0}\right|=k$. Notice that $\bigcap_{U \in \mathcal{U}_{0}} \partial U^{\prime}$ is a subcomplex of $Z^{(m+a)}$ for some $a$ since the intersection of a subcomplex of $Z^{(n+a)}$ with a subcomplex of $Z^{(n+b)}$ is a subcomplex of $Z^{(n+b)}$ if $a \leq b$. It suffices to show $\operatorname{dim}\left(\bigcap_{U \in \mathcal{U}_{0}} \partial U^{\prime}\right) \leq n-k$ since this implies

$$
\bigcap_{U \in \mathcal{U}_{0}} \partial U^{\prime}=\varnothing \quad \text { if } \mathcal{U}_{0} \text { contains more than } n \text { elements. }
$$

Choose $i_{1}, i_{2}, \ldots, i_{k}$ in $\{1,2, \ldots, r\}$ and $g_{1}, g_{2}, \ldots, g_{k}$ in $F$ with the property that $\mathcal{U}_{0}$ consists of the mutually different elements $g_{1} U_{i_{1}}, g_{2} U_{i_{2}}, \ldots, g_{k} U_{i_{k}}$ and $i_{1} \leq$ $i_{2} \leq \ldots \leq i_{k}$ holds. If for some $j \in\{1,2, \ldots,(r-1)\}$ we have $i_{j}=i_{j+1}$, then $g_{j} U_{i_{j}} \neq g_{j+1} U_{i_{j+1}}$ implies already $g_{j} U_{i_{j}} \cap g_{j+1} U_{i_{j+1}}=\varnothing$ and the claim is obviously true. Hence we can assume without loss of generality $i_{1}<i_{2}<\ldots<i_{k}$. Next we show by induction for $j=1,2, \ldots, k$ that

$$
\operatorname{dim}\left(\bigcap_{l=1}^{j} \partial g_{i_{l}} U_{i_{l}}^{\prime}\right) \leq n-j .
$$

The induction beginning is obvious since the dimension of the boundary of a simplicial complex is smaller than the dimension of the simplicial complex itself. The induction step from $j-1$ to $j$ is done as follows. By induction hypothesis the dimension of the simplicial subcomplex $\bigcap_{l=1}^{j-1} \partial g_{i_{l}} U_{i_{l}}^{\prime}$ of $Z^{\left(n+i_{j-1}\right)}$ is less or equal to $(n-j+1)$. Let $\sigma$ be a simplex of the subcomplex $\bigcap_{l=1}^{j} \partial g_{i_{l}} U_{i_{l}}^{\prime}$ of $Z^{\left(n+i_{j}\right)}$. We can find a simplex $\beta$ in $\bigcap_{l=1}^{j-1} \partial g_{i_{l}} U_{i_{l}}^{\prime}$ such that $\sigma$ is contained in $\beta$. Recall that by assumption $U_{j}$ is the interior of a subcomplex $B_{j}$ of $Z^{\left(n+i_{j-1}\right)}$. Proposition 3.10 applied to the case $m=i_{j}-i_{j-1}$ and $B=g_{j} B_{j}$ and $\sigma$ and $\beta$ as above implies

$$
\operatorname{dim}(\sigma)<\operatorname{dim}(\beta) \leq n-j+1
$$

since in the notation of Proposition 3.10 we have $B_{0}=g_{j} U_{i_{j}}^{\prime}$. Hence $\operatorname{dim}(\sigma) \leq n-j$. This finishes the proof of Proposition 3.2 in the special case where $Z$ is a simplicial complex, each $U \in \mathcal{U}$ is the interior of a subcomplex of $Z$ and $F$ acts simplicially on $Z$.

In the general case we start with an open cover $\mathcal{V}$ of $Z$ such that each $V \in \mathcal{V}$ has diameter $<\epsilon=\delta / 3$ and $\operatorname{dim}(\mathcal{V}) \leq n$. Let $\mathcal{V}_{F}$ be the cover of $Z$ whose members are 
the open sets of the form $g V$ with $V \in \mathcal{V}$ and $g \in F$. Then $\mathcal{V}_{F}$ is an open cover of $Z$ whose members have diameter $<\epsilon$ and we have $g V \in \mathcal{V}_{F}$ for $V \in \mathcal{V}_{F}$ and $g \in F$. Analogously as in Proposition 3.3 one shows that $\operatorname{dim}\left(\mathcal{V}_{F}\right) \leq m$.

For $U \in \mathcal{U}$ let $\mathcal{V}_{U}=\left\{V \in \mathcal{V}_{F} \mid V \subseteq U\right\}$ and consider $\mathcal{N}\left(\mathcal{V}_{U}\right)$ as a subcomplex of $\mathcal{N}\left(\mathcal{V}_{F}\right)$ (this is the subcomplex spanned by the vertices [V] with $\left.V \in \mathcal{V}_{U}\right)$ and denote by $\hat{U}$ the interior of $\mathcal{N}\left(\mathcal{V}_{U}\right)$. Consider the map $f=f_{\mathcal{V}_{F}}: Z \rightarrow \mathcal{N}\left(\mathcal{V}_{F}\right)$ from Remark 3.4. If $x \in f^{-1}\left(\mathcal{N}\left(\mathcal{V}_{U}\right)\right)$ and $x \in V, V \in \mathcal{V}_{F}$ then by the construction of $f, V \in \mathcal{V}_{U}$. Therefore

$$
f^{-1}(\widehat{U}) \subseteq f^{-1}\left(\mathcal{N}\left(\mathcal{V}_{U}\right)\right) \subseteq U
$$

Let $x \in U^{-\epsilon}$. If $x \in V$ with $V \in \mathcal{V}_{F}$ then $V \in \mathcal{V}_{U}$, because the diameter of $V$ is $<\epsilon$. Therefore $f(x) \in \mathcal{N}\left(\mathcal{V}_{U}\right)$. If $f(x)$ lies on the boundary of $\mathcal{N}\left(\mathcal{V}_{U}\right)$, then there are $V \in \mathcal{V}_{U}, V^{\prime} \in \mathcal{V}_{F}-\mathcal{V}_{U}$ such that $x \in V$ and $V \cap V^{\prime} \neq \varnothing$. In particular this implies $x \notin U^{-2 \epsilon}$. We have thus shown that

$$
U^{-2 \epsilon} \subseteq f^{-1}(\widehat{U}) \subseteq U .
$$

Equip $\mathcal{N}\left(\mathcal{V}_{F}\right)$ with a metric such that the action of $F$ is by isometries. By Lemma 3.6 there is $\alpha$ such that

$$
\left(f^{-1}(\widehat{U})\right)^{-\epsilon} \subseteq f^{-1}\left(\hat{U}^{-\alpha}\right)
$$

for all $U \in \mathcal{U}$. By the special case treated in the first part of this proof for each $\widehat{U}$ there is $\hat{U}^{\prime}$ such that

$$
\begin{aligned}
& (g \hat{U})^{\prime}=g\left(\hat{U}^{\prime}\right) \text { for } g \in F \text { and } \\
& \bigcap_{U \in \hat{U}_{0}-\alpha} \partial \hat{U}^{\prime}=\varnothing \quad \text { if } \mathcal{U}_{0} \text { contains more than } m \text { elements. }
\end{aligned}
$$

(See (3.11) and recall that $\operatorname{dim}\left(\mathcal{V}_{F}\right) \leq m$.) Finally set $U^{\prime}=f^{-1}\left(\widehat{U}^{\prime}\right)$ for $U \in \mathcal{U}$. Since $\partial f^{-1}(U) \subseteq f^{-1}(\partial U)$ and taking preimages preserves inclusions and intersections (ii) and (iii) are satisfied. Moreover, by (3.12) and (3.13)

$$
U^{-\delta}=U^{-3 \epsilon} \subseteq\left(f^{-1}(\widehat{U})\right)^{-\epsilon} \subseteq f^{-1}\left(\widehat{U}^{-\alpha}\right) \subseteq f^{-1}\left(\hat{U}^{\prime}\right) \subseteq f^{-1}(\widehat{U}) \subseteq U
$$

and therefore (i) is also satisfied.

\section{Covering $X_{>\gamma}$ by long boxes}

Throughout this Section we will assume that we are in the situation of Convention 1.3. In particular $k_{G}$ is the maximum over the orders of finite subgroups of $G$ and 
$d_{X}$ is the dimension of $X-X^{\mathbb{R}}$. Both $k_{G}$ and $d_{X}$ are finite. Also recall the notation $X_{>r}$ introduced in Definition 2.15. This section is entirely devoted to the proof of the following

Proposition 4.1 There exists a natural number $M=M\left(k_{G}, d_{X}\right)$ depending only on $k_{G}$ and $d_{X}$ which has the following property:

For every $\alpha, \epsilon \in \mathbb{R}$ with $0<\epsilon<\alpha$ there exists $\gamma=\gamma(\alpha, \epsilon, M)>0$ such that for every cocompact $G$-invariant subset $K$ of $X_{>\gamma}$, there is a collection $\mathcal{D}$ of boxes satisfying:

(4.2) $K \subseteq \bigcup_{D \in \mathcal{D}} \Phi_{(-\epsilon, \epsilon)}\left(D^{\circ}\right)$;

(4.3) For every $x \in X$ which lies on the open bottom or open top of a box in $\mathcal{D}$, the set $\Phi_{[-\alpha,-\epsilon] \cup[\epsilon, \alpha]}(x)$ does not intersect the open bottom or the open top of a box in $\mathcal{D}$;

(4.4) For any $x \in X$ there is no box $D \in \mathcal{D}$ such that $\Phi_{[0, \alpha]}(x)$ intersects both the open bottom and open top of $D$;

(4.5) The dimension of the collection $\left\{D^{\circ} \mid D \in \mathcal{D}\right\}$ is less or equal to $M$, ie the intersection of $(M+2)$ pairwise distinct elements is always empty;

(4.6) For $g \in G, D \in \mathcal{D}$ we have $g D \in \mathcal{D}$;

(4.7) There is a finite subset $\mathcal{D}_{0} \subseteq \mathcal{D}$ such that for every $D \in \mathcal{D}$ there exists $g \in G$ with $g D \in \mathcal{D}_{0}$; $\Phi_{[-\alpha-\epsilon, \alpha+\epsilon]}(D)$ is a $\mathcal{F}$ in-subset of $X$ for all $D \in \mathcal{D}$.

The idea of the proof is very roughly as follows. Conditions (4.2) and (4.3) require the boxes to be very long, but we have still the freedom to make the boxes very thin. Proposition 3.2 applied to the transversal directions will be important to arrange the boxes during the construction to be in general position. This will allow the application of Proposition 3.3, where property (iii) will be crucial in order control how many boxes from previous steps of the construction interfere at each step in the construction.

\subsection{Preliminaries and the basic induction structure}

We begin with fixing some numbers and collection of boxes. Define numbers

$$
\begin{aligned}
m & :=k_{G} \cdot\left(d_{X}+1\right) \\
M & :=\left(k_{G}\right)^{2} \cdot\left(d_{X}+1\right)+2^{m+1} ; \\
a & :=\epsilon / 2 \\
b & :=4 M \cdot(\alpha+2 \epsilon)+3(\alpha+\epsilon) \\
c & :=a+2(b+\epsilon)+1+\epsilon ; \\
\gamma & :=a+2 b+2 c .
\end{aligned}
$$


Notice that $m$ and $M$ depend only on $k_{G}$ and $d_{X}$ and all the other numbers depend only on $\alpha, \epsilon$ and $M$. (The reader may wonder why we picked $a$ small, we are after all looking for long boxes. But for our construction it is only important that $b$ and $c$ are large and in the proof of Lemma 4.49 (iii) our choice of a small $a$ will be convenient.)

Let $A_{\lambda} \subseteq B_{\lambda} \subseteq C_{\lambda}$ for $\lambda \in \Lambda$ be three collection of boxes as in the assertion of Lemma 2.17 , where we use $a$ as defined above and replace $b$ by $b+\epsilon$, and $c$ by $c-\epsilon$. Then we replace $B_{\lambda}$ by the restriction $B_{\lambda}(a+2 b)$. The resulting collections satisfy:

- $\Lambda$ is $G$-cofinite;

- $l_{A}=a, l_{B}=a+2 b$ and $l_{C}=a+2 b+2 c$;

- $S_{C_{\lambda}}$ is connected;

- $S_{A_{\lambda}} \subseteq S_{B_{\lambda}} \subseteq S_{C_{\lambda}}$;

- $A_{\lambda} \subseteq B_{\lambda}^{\circ}$ and $B_{\lambda} \subseteq C_{\lambda}^{\circ}$;

- $K \subseteq \bigcup_{\lambda \in \Lambda} A_{\lambda}^{\circ}$;

- $g A_{\lambda}=A_{g \lambda}, g B_{\lambda}=B_{g \lambda}$ and $g C_{\lambda}=C_{g \lambda}$ for $g \in G$ and $\lambda \in \Lambda$;

- If $B_{\lambda} \cap B_{\lambda^{\prime}} \neq \varnothing$, then $\phi_{[-\epsilon, \epsilon]}\left(B_{\lambda}\right) \subseteq C_{\lambda^{\prime}}^{\circ}$ and $S_{B_{\lambda}}$ is transversal to the flow with respect to $C_{\lambda^{\prime}}$.

Next we discuss the main strategy of the proof. We will construct the desired collection $\mathcal{D}$ inductively over larger and larger parts of $K$. The boxes $C_{\lambda}$ will be used to control the group action, ie, they will allow us to restrict attention to the finite group $G_{C_{\lambda}}$. The boxes $B_{\lambda}$ will be used to control properties (4.2) and (4.3). The boxes $A_{\lambda}$ will be used to control which part of $K$ is already covered. We will need to sharpen the induction assumptions. We introduce a minor but useful variation of (4.2) as follows.

Definition 4.9 A collection of boxes is $\delta$-overlong for $0 \leq \delta<\epsilon$ if for every $x \in$ $X$ which lies on the open bottom or open top of a box in this collection, the set $\Phi_{[-\alpha-\delta,-\epsilon+\delta] \cup[\epsilon-\delta, \alpha+\delta]}(x)$ does not intersect the open bottom or the open top of any box in this collection.

The assertion (4.2) is then that $\mathcal{D}$ is 0 -overlong. Clearly, $\delta$-overlong for some $0 \leq \delta<\epsilon$ implies $0-$ overlong.

Definition 4.10 We say that a box $D$ is not huge if for every $\lambda \in \Lambda$ such that $D$ intersects $B_{\lambda}$ we have $\phi_{[-\epsilon, \epsilon]}(D) \subset C_{\lambda}^{\circ}$ and $S_{D}$ is transversal to the flow with respect to $C_{\lambda}$. 
Every box which is obtained from one of the boxes $B_{\lambda}$ by restriction is automatically not huge.

Definition 4.11 We say a collection $\mathcal{E}$ of boxes is a $\delta$-good box cover of a subset $S$ of $X$ if it has the following properties:

- $\mathcal{E}$ is $\delta$-overlong;

- Every box in $\mathcal{E}$ is not huge;

- Assertions (4.3), (4.4), (4.5), (4.6) and (4.7) hold for $\mathcal{E}$ in place of $\mathcal{D}$;

- $S \subseteq \bigcup_{E \in \mathcal{E}} \Phi_{(-\epsilon, \epsilon)}\left(E^{\circ}\right)$.

To prove Proposition 4.1 we will construct a $0-\operatorname{good}$ box cover of $K$. Let $N$ be the number of $G$-orbits of $\Lambda$.

Put

$$
\delta_{r}=\frac{N-r}{N+1} \cdot \epsilon \quad \text { for } \quad r=0,1, \ldots, N
$$

Clearly,

$$
\epsilon>\frac{N}{N+1} \epsilon=\delta_{0}>\cdots>\delta_{r}>\delta_{r+1}>\cdots>\delta_{N}=0 .
$$

We will show inductively for $r=0,1,2, \ldots, N$ that for any subset $\Xi \subseteq \Lambda$ consisting of $r G$-orbits there exists a $\delta_{r}$-good box cover of $K_{\Xi}:=\bigcup_{\xi \in \Xi} A_{\xi}$. The induction beginning $r=0$ is trivial, take $\mathcal{D}=\varnothing$. The induction step from $r$ to $r+1$ is summarized in the next lemma.

Lemma 4.13 (Induction step : $r$ to $r+1$ ) Let $\Xi \subseteq \Lambda$ consist of $r G$-orbits and assume that $\mathcal{D}$ is a $\delta_{r}$-good box cover of $K_{\Xi}=\bigcup_{\xi \in \Xi} A_{\xi}$. Let $\lambda \in \Lambda-\Xi$ and $\Xi^{\prime}:=G \lambda \cup \Xi$. Then there is a $\delta_{r+1}-\operatorname{good}$ box cover $\mathcal{D}^{\prime}$ of $K_{\Xi^{\prime}}=\bigcup_{\xi \in \Xi^{\prime}} A_{\xi}$.

Clearly Lemma 4.13 implies Proposition 4.1. The proof of Lemma 4.13 will occupy the remainder of Section 4.

\subsection{Boxes in $C_{\lambda}^{\circ}$}

Before we explain the construction of $\mathcal{D}^{\prime}$, we have to introduce some notation and to rearrange $\mathcal{D}$ as follows. In the sequel everything will take place in the interior of the box $C_{\lambda}$. Recall for the sequel the $G_{C_{\lambda}}$-homeomorphism

$$
\mu_{C_{\lambda}}: S_{C_{\lambda}} \times[-a / 2-b-c, a / 2+b+c] \rightarrow C_{\lambda}, \quad(x, \tau) \mapsto \Phi_{\tau}(x)
$$

from Lemma 2.6 (iii). Let $\pi_{C_{\lambda}}: C_{\lambda} \rightarrow S_{C_{\lambda}}, x \mapsto \Phi_{\left(a_{+}(x)+a_{-}(x)\right) / 2}(x)$ be the retraction onto the central slice. Closures and interiors of subsets of $S_{C_{\lambda}}$ are always understood with respect to $S_{C_{\lambda}}$. 
Definition 4.14 Let $E \subseteq C_{\lambda}^{\circ}$ be a box such that $S_{E}$ is transversal to the flow with respect to $C_{\lambda}$.

Define an open subset of $S_{C_{\lambda}} \cap C_{\lambda}^{\circ}$ by

$$
U_{E}:=\pi_{C_{\lambda}}\left(S_{E} \cap E^{\circ}\right)=\pi_{C_{\lambda}}\left(E^{\circ}\right) .
$$

Define the continuous $G_{E}$-invariant map

$$
\tau_{E}: \pi_{C_{\lambda}}\left(S_{E}\right) \rightarrow\left[-l_{C} / 2, l_{C} / 2\right]
$$

to be the composite of the inverse of $\left.\pi_{C_{\lambda}}\right|_{S_{E}}: S_{E} \cong \pi_{C_{\lambda}}\left(S_{E}\right)$, the inverse of $\mu_{C_{\lambda}}$ restricted to $S_{E}$ and the projection $S_{C_{\lambda}} \times\left[-l_{C} / 2, l_{C} / 2\right] \rightarrow\left[-l_{C} / 2, l_{C} / 2\right]$.

For a subset $T \subseteq S_{C_{\lambda}}$ define a subset of $S_{E}$ by

$$
\sigma_{E}(T):=\pi_{C_{\lambda}}^{-1}(T) \cap S_{E}
$$

Lemma 4.15 Let $E, E^{\prime} \subseteq C_{\lambda}^{\circ}$ be boxes such that $S_{E}$ and $S_{E^{\prime}}$ are transversal to the flow with respect to $C_{\lambda}$. Then:

(i) If $g E \cap E^{\prime} \neq \varnothing$ for some $g \in G$, then $g \in G_{C_{\lambda}}$. In particular $G_{E}$ is a subgroup of $G_{C_{\lambda}}$;

(ii) The map $\tau_{E}$ is uniquely characterized by

$$
\mu_{C_{\lambda}}(x)=\left(\pi_{C_{\lambda}}(x), \tau_{E} \circ \pi_{C_{\lambda}}(x)\right)
$$

for $x \in S_{E}$;

(iii) If $T \subseteq S_{C_{\lambda}}$ is a closed $\mathcal{F}$ in-subset of the $G_{E}$-space $S_{C_{\lambda}}$, then $\sigma_{E}(T)$ is a closed $\mathcal{F}$ in-subset of the $G_{E}$-space $S_{E}$ and the restriction $E\left(\sigma_{E}(T)\right)$ is defined;

(iv) Let $U \subseteq S_{C_{\lambda}}$ be an open $\mathcal{F}$ in-subset of the $G_{E}$-space $S_{C_{\lambda}}$ with $\bar{U} \subseteq \pi_{C_{\lambda}}\left(S_{E}\right)$ and $U=\bar{U}^{\circ}$. Then

$$
\begin{aligned}
E\left(\sigma_{E}(\bar{U})\right) & =\Phi_{\left[-l_{E} / 2, l_{E} / 2\right]}\left(\sigma_{E}(\bar{U})\right) ; \\
E\left(\sigma_{E}(\bar{U})\right)^{\circ} & =\Phi_{\left(-l_{E} / 2, l_{E} / 2\right)}\left(\sigma_{E}(U)\right) ; \\
U_{E\left(\sigma_{E}(\bar{U})\right)} & =U .
\end{aligned}
$$

Proof (i) Suppose $g E \cap E^{\prime} \neq \varnothing$. Since then $g C_{\lambda} \cap C_{\lambda} \neq \varnothing$, we get $g \in G_{C_{\lambda}}$.

(ii) This follows from the definitions.

(iii) This is obvious since $\pi_{C_{\lambda}}$ is $G_{E}$-equivariant. 
(iv) We conclude $E\left(\sigma_{E}(\bar{U})\right)=\Phi_{\left[-l_{E} / 2, l_{E} / 2\right]}\left(\sigma_{E}(\bar{U})\right)$ from the definition of the restriction. The set $\Phi_{\left(-l_{E} / 2, l_{E} / 2\right)}\left(\sigma_{E}(U)\right)$ is mapped under the homeomorphism $\mu_{C_{\lambda}}$ to the set $\left\{(u, t)|u \in U| t-,\tau_{E}(u) \mid<l_{C} / 2\right\}$. Since $\tau_{E}$ is continuous and $U$ is open in $S_{C_{\lambda}}$, this set and hence $\Phi_{\left(-l_{E} / 2, l_{E} / 2\right)}\left(\sigma_{E}(U)\right) \subseteq C_{\lambda}^{\circ}$ are open. This implies

$$
\begin{aligned}
\Phi_{\left(-l_{E} / 2, l_{E} / 2\right)}\left(\sigma_{E}(U)\right) & \subseteq E\left(\sigma_{E}(\bar{U})\right)^{\circ} ; \\
\sigma_{E}(U) & \subseteq \sigma_{E}(\bar{U}) \cap E\left(\sigma_{E}(\bar{U})\right)^{\circ} .
\end{aligned}
$$

If we apply $\pi_{C_{\lambda}}$ to the latter inclusion, we conclude

$$
U \subseteq \bar{U} \cap \pi_{C_{\lambda}}\left(E\left(\sigma_{E}(\bar{U})\right)^{\circ}\right) \subseteq \bar{U}^{\circ}=U .
$$

This implies $\sigma_{E}(U)=\sigma_{E}(\bar{U}) \cap E\left(\sigma_{E}(\bar{U})\right)^{\circ}$ and $U_{E\left(\sigma_{E}(\bar{U})\right)}=U$. Lemma 2.6 (iii) implies $\Phi_{\left(-l_{E} / 2, l_{E} / 2\right)}\left(\sigma_{E}(U)\right)=E\left(\sigma_{E}(\bar{U})\right)^{\circ}$.

\subsection{Rearranging the data of the induction beginning}

Let $\mathcal{D}$ be the collection of boxes, from the hypothesis of Lemma 4.13 (with respect to $\Xi \subset \Lambda$ and $\lambda \in \Lambda-\Xi)$.

Definition 4.16 Put

$$
\begin{aligned}
\mathcal{D}_{\lambda} & :=\left\{D \in \mathcal{D} \mid D \cap B_{\lambda} \neq \varnothing\right\} ; \\
\mathcal{U}\left(D_{\lambda}\right) & :=\left\{U_{D} \mid D \in \mathcal{D}_{\lambda}\right\} .
\end{aligned}
$$

Since the $G$-action on $X$ is proper and $B_{\lambda}$ is compact, property (4.6) implies that $\mathcal{D}_{\lambda}$ is finite. We will consider the $G_{C_{\lambda}}$ sets

$$
\begin{aligned}
G_{C_{\lambda}} \cdot \mathcal{D}_{\lambda} & =\left\{g D \mid D \in \mathcal{D}_{\lambda}, g \in G_{C_{\lambda}}\right\} \\
G_{C_{\lambda}} \cdot \mathcal{U}\left(\mathcal{D}_{\lambda}\right) & =\left\{g U_{D} \mid D \in \mathcal{D}_{\lambda}, g \in G_{C_{\lambda}}\right\}=\left\{U_{E} \mid E \in G_{C_{\lambda}} \cdot \mathcal{D}_{\lambda}\right\} .
\end{aligned}
$$

Since every $D \in \mathcal{D}_{\lambda}$ is not huge, the set $D$ is contained in $C_{\lambda}^{\circ}$ and $S_{D}$ is transversal to the flow with respect to $C_{\lambda}$. Both these properties also hold for every $D \in G_{C_{\lambda}} \cdot \mathcal{D}_{\lambda}$.

We use Proposition 3.2 to diminish the elements in $\mathcal{D}$ slightly in order to obtain a general position property for $\mathcal{D}$. At this point it is important, that we arranged the central slice $S_{C_{\lambda}}$ to be connected.

We main goal of this subsection will be the proof of the following lemma. 
Lemma 4.17 We can assume without loss of generality that $\mathcal{D}$ has the following general position properties:

$$
\bigcap_{U \in \mathcal{U}_{0}} \partial U=\varnothing \text { when } \mathcal{U}_{0} \subseteq G_{C_{\lambda}} \cdot \mathcal{U}\left(\mathcal{D}_{\lambda}\right) \text { fulfills }\left|\mathcal{U}_{0}\right|>m=k_{G} \cdot\left(d_{X}+1\right) ;
$$

If $D, D^{\prime} \in G_{C_{\lambda}} \cdot \mathcal{D}_{\lambda}$ and $U_{D}=U_{D^{\prime}}$ then $D=D^{\prime}$.

The proof of Lemma 4.17 will use the following lemma, that we will prove first.

Lemma 4.20 There exists collections $\left\{V_{D} \mid D \in G_{C_{\lambda}} \cdot \mathcal{D}_{\lambda}\right\}$ and $\left\{W_{D} \mid D \in G_{C_{\lambda}} \cdot \mathcal{D}_{\lambda}\right\}$ of open subsets of $S_{C_{\lambda}}$ satisfying:

(i) For $D \in G_{C_{\lambda}} \cdot \mathcal{D}_{\lambda}$ the sets $W_{D}, \overline{W_{D}}, V_{D}$ and $\overline{V_{D}}$ are $G_{D}$-invariant subsets of the $G_{D}$-space $U_{D}$;

(ii) We have $W_{D} \subseteq \overline{W_{D}} \subseteq V_{D} \subseteq \overline{V_{D}} \subseteq U_{D}$ for $D \in G_{C_{\lambda}} \cdot \mathcal{D}_{\lambda}$;

(iii) We have $W_{D}=\left(\overline{W_{D}}\right)^{\circ}$ and $V_{D}=\left(\overline{V_{D}}\right)^{\circ}$ for $D \in G_{C_{\lambda}} \cdot \mathcal{D}_{\lambda}$;

(iv) $W_{g D}=g W_{D}$ and $V_{g D}=g V_{D}$ holds for $g \in G_{C_{\lambda}}$ and $D \in G_{C_{\lambda}} \cdot \mathcal{D}_{\lambda}$;

(v) $K_{\Xi} \subseteq \bigcup_{\substack{D \in \mathcal{D} \\ D \notin G \cdot \mathcal{D}_{\lambda}}} \Phi_{(-\epsilon, \epsilon)}\left(D^{\circ}\right) \cup \bigcup_{g \in G} \bigcup_{D \in \mathcal{D}_{\lambda}} g \cdot \Phi_{(-\epsilon, \epsilon)}\left(D\left(\sigma_{D}\left(\overline{W_{D}}\right)\right)^{\circ}\right)$;

(vi) If $V_{D}=V_{E}$ for $D, E \in G_{C_{\lambda}} \cdot \mathcal{D}_{\lambda}$, then $D=E$;

(vii) If for $D, E \in G_{C_{\lambda}} \cdot \mathcal{D}_{\lambda}$ the intersection $V_{D} \cap V_{E}$ contains both $W_{D}$ and $W_{E}$, then $V_{D}=V_{E}$.

Proof Choose a metric $d$ on $S_{C_{\lambda}}$ which is $G_{C_{\lambda}}$-invariant. Consider $D \in G_{C_{\lambda}} \cdot \mathcal{D}_{\lambda}$. Put

$$
V_{D}(n)=\left(\overline{U_{D}^{-1 / n}}\right)^{\circ} \text {. }
$$

Notice for the sequel that for an open subset $Y$ of a topological space we have $\bar{Y}=\overline{\bar{Y}^{\circ}}$ and $Y \subseteq \bar{Y}^{\circ}$ but in general $Y \neq \bar{Y}^{\circ}$. Hence $V_{D}(n)$ is a $G_{D}$-invariant open subset of $S_{C_{\lambda}}$ with $V_{D}(n)=\left(\overline{V_{D}(n)}\right)^{\circ}$. We get for $D \in G_{C_{\lambda}} \cdot \mathcal{D}_{\lambda}$ and $g \in G_{C_{\lambda}}$ that $\overline{V_{D}(n)} \subseteq V_{D}(n+1) \subseteq U_{D}, U_{D}=\bigcup_{n \geq 1} V_{D}(n)$ and $g V_{D}(n)=V_{g D}(n)$ holds. Denote for $D \in G_{C_{\lambda}} \cdot \mathcal{D}_{\lambda}$ and $n \geq 1$ the restriction by

$$
D_{n}=D\left(\sigma_{D}\left(\overline{V_{D}(n)}\right), l_{D}-1 / n\right) .
$$

Lemma 4.15 (iv) and Lemma 2.6 (iii) imply $D^{\circ}=\bigcup_{n \geq 1} D_{n}^{\circ}$ and $D_{n} \subseteq D_{n+1}^{\circ}$ for $n \geq 1$.

Put

$$
K_{\Xi}^{\prime}:=K_{\Xi}-\left(K_{\Xi} \cap \bigcup_{\substack{D \in \mathcal{D} \\ D \notin G \cdot \mathcal{D}_{\lambda}}} \Phi_{(-\epsilon, \epsilon)}\left(D^{\circ}\right)\right) .
$$


Since $K_{\Xi} \subseteq \bigcup_{D \in \mathcal{D}} \Phi_{(-\epsilon, \epsilon)}\left(D^{\circ}\right)$ by assumption, we have

$$
\begin{aligned}
& K_{\Xi}^{\prime} \subseteq \bigcup_{D \in G \cdot \mathcal{D}_{\lambda}} \Phi_{(-\epsilon, \epsilon)}\left(D^{\circ}\right) ; \\
& K_{\Xi} \subseteq K_{\Xi}^{\prime} \cup \bigcup_{\substack{D \in \mathcal{D} \\
D \notin G \cdot \mathcal{D}_{\lambda}}} \Phi_{(-\epsilon, \epsilon)}\left(D^{\circ}\right) .
\end{aligned}
$$

Since each $D \in \mathcal{D}$ is not huge, $\Phi_{[-\epsilon, \epsilon]}(D)$ is contained in $C_{\lambda}^{\circ}$ for $D \in \mathcal{D}_{\lambda}$. Since $g C_{\lambda} \cap C_{\lambda} \neq \varnothing \Rightarrow g \in G_{C_{\lambda}}$, we get from (4.21)

$$
\begin{aligned}
K_{\Xi}^{\prime} \cap C_{\lambda} & \subseteq \bigcup_{D \in G_{C_{\lambda}} \cdot \mathcal{D}_{\lambda}} \Phi_{(-\epsilon, \epsilon)}\left(D^{\circ}\right) ; \\
K_{\Xi}^{\prime} & =\bigcup_{g \in G} g \cdot\left(K_{\Xi}^{\prime} \cap C_{\lambda}\right) .
\end{aligned}
$$

Since $K_{\Xi}^{\prime}$ is closed, $K_{\Xi}^{\prime} \cap C_{\lambda}$ is compact. Because $D^{\circ}=\bigcup_{n \geq 1} D_{n}^{\circ}$ and $D_{n}^{\circ} \subset D_{n+1}^{\circ}$, (4.23) implies that there exists a natural number $N$ with

$$
K_{\Xi}^{\prime} \cap C_{\lambda} \subseteq \bigcup_{D \in G_{C_{\lambda}} \cdot \mathcal{D}_{\lambda}} \Phi_{(-\epsilon, \epsilon)}\left(D_{N}^{\circ}\right)
$$

Since $D_{N} \subseteq D\left(\sigma_{D}\left(\overline{V_{D}(N)}\right)\right.$ we conclude

$$
K_{\Xi}^{\prime} \cap C_{\lambda} \subseteq \bigcup_{D \in G_{C_{\lambda}} \cdot \mathcal{D}_{\lambda}} \Phi_{(-\epsilon, \epsilon)}\left(D\left(\sigma_{D}\left(\overline{V_{D}(N)}\right)^{\circ}\right) .\right.
$$

We conclude from (4.24)

$$
K_{\Xi}^{\prime} \subseteq \bigcup_{g \in G} \bigcup_{D \in \mathcal{D}_{\lambda}} g \cdot \Phi_{(-\epsilon, \epsilon)}\left(D\left(\sigma_{D}\left(\overline{V_{D}(N)}\right)^{\circ}\right) .\right.
$$

We conclude from (4.22)

$$
K_{\Xi} \subseteq \bigcup_{\substack{D \in \mathcal{D} \\ D \notin G \cdot \mathcal{D}_{\lambda}}} \Phi_{(-\epsilon, \epsilon)}\left(D^{\circ}\right) \cup \bigcup_{g \in G} \bigcup_{D \in \mathcal{D}_{\lambda}} g \cdot \Phi_{(-\epsilon, \epsilon)}\left(D\left(\sigma_{D}\left(\overline{V_{D}(N)}\right)\right)^{\circ}\right) .
$$

So for every choice of natural numbers $\left\{n_{D} \mid D \in G_{C_{\lambda}} \cdot \mathcal{D}_{\lambda}\right\}$ satisfying $n_{D} \geq N$ and $n_{g D}=n_{D}$ for $g \in G_{C_{\lambda}}$ and $D \in G_{C_{\lambda}} \cdot \mathcal{D}_{\lambda}$ the collection $\left\{V_{D}\left(n_{D}\right) \mid D \in G_{C_{\lambda}} \cdot \mathcal{D}_{\lambda}\right\}$ has the following properties:

- $V_{D}\left(n_{D}\right)$ is a $G_{D}$-invariant subset of the $G_{D}$-space $V_{D}$;

- $V_{D}\left(n_{D}\right)=\left(\overline{V_{D}\left(n_{D}\right)}\right)^{\circ}$;

- We have $V_{D}\left(n_{D}\right) \subseteq \overline{V_{D}\left(n_{D}\right)} \subseteq U_{D}$ for $D \in G_{C_{\lambda}} \cdot \mathcal{D}_{\lambda}$; 
- $V_{g D}\left(n_{g D}\right)=g V_{D}\left(n_{D}\right)$ holds for $g \in G_{C_{\lambda}}$ and $D \in G_{C_{\lambda}} \cdot \mathcal{D}_{\lambda}$;

- $K_{\Xi} \subseteq \bigcup_{\substack{D \in \mathcal{D} \\ D \notin G \cdot \mathcal{D}_{\lambda}}} \Phi_{(-\epsilon, \epsilon)}\left(D^{\circ}\right) \cup \bigcup_{g \in G} \bigcup_{D \in \mathcal{D}_{\lambda}} g \cdot \Phi_{(-\epsilon, \epsilon)}\left(D\left(\sigma_{D}\left(\overline{V_{D}\left(n_{D}\right)}\right)\right)^{\circ}\right)$.

Next we show that for some choice of numbers $\left\{n_{D} \mid D \in G_{C_{\lambda}} \cdot \mathcal{D}_{\lambda}\right\}$ satisfying $n_{D} \geq N$ and $n_{g D}=n_{D}$ for $g \in G_{C_{\lambda}}$ and $D \in G_{C_{\lambda}} \cdot \mathcal{D}_{\lambda}$ the collection $\left\{V_{D}\left(n_{D}\right) \mid D \in G_{C_{\lambda}} \cdot \mathcal{D}_{\lambda}\right\}$ also has property (vi). We can write $G_{C_{\lambda}} \cdot \mathcal{D}_{\lambda}$ as the disjoint union

$$
G_{C_{\lambda}} \cdot \mathcal{D}_{\lambda}=\mathcal{C}_{1} \amalg \mathcal{C}_{2} \amalg \ldots \amalg \mathcal{C}_{r}
$$

of its $G_{C_{\lambda}}$-orbits. We show by induction that we can find numbers $n_{1}, n_{2}, \ldots, n_{r}$ with $n_{k} \geq N$ such that if we set $n_{D}=n_{k}$ for $D \in \mathcal{C}_{k}$ the collection $\left\{V_{D}=V_{D}\left(n_{D}\right) \mid D \in\right.$ $\left.\mathcal{C}_{1} \cup \mathcal{C}_{2} \cup \cdots \cup \mathcal{C}_{k}\right\}$ satisfies property (vi). The induction beginning $k=1$ is trivial, the induction step from $k-1$ to $k$ is done as follows. For given $D \in \mathcal{C}_{k}$ choose $n_{0}$ with $\overline{V_{D}\left(n_{0}\right)} \neq \varnothing$. Since $S_{C_{\lambda}}$ is connected, the nonempty closed subset $\overline{V_{D}(n)}$ and the open subset $V_{D}(n+1) \neq S_{C_{\lambda}}$ cannot agree for $n \geq n_{0}$. In particular $V_{D}(n) \subset V_{D}(n+1)$ for all $n \geq n_{0}$ and since $\mathcal{C}_{1} \cup \mathcal{C}_{2} \cup \cdots \cup \mathcal{C}_{k-1}$ is a finite set we can find a number $n_{k}$ such that $V_{D}\left(n_{k}\right) \neq V_{E}\left(n_{E}\right)$ for $E \in \mathcal{C}_{1} \cup \mathcal{C}_{2} \cup \cdots \cup \mathcal{C}_{k-1}$. By invariance under the $G_{C_{\lambda}}$-action the same statement holds for all $D \in \mathcal{C}_{k}$ with this $n_{k}$. If we have $D, g D \in \mathcal{C}_{k}$ with $g \in G_{C_{\lambda}}$ then since $\mu_{C_{\lambda}}$ from Lemma 2.6 (iii) is $G_{C_{\lambda}}$-invariant and the action on the interval trivial and $D$ is a $\mathcal{F}$ in-set, $U_{D}=g U_{D}$ already implies $D=g D$. Therefore property (vi) holds for $\mathcal{C}_{1} \cup \mathcal{C}_{2} \cup \cdots \cup \mathcal{C}_{k}$. We have therefore verified property (vi) for the collection $V_{D}=V_{D}\left(n_{D}\right)$ with $D \in G_{C_{\lambda}} \mathcal{D}_{\lambda}$.

In order to achieve property (vii) we repeat this construction replacing the collection of boxes $G_{C_{\lambda}} \mathcal{D}_{\lambda}$ with the collection of boxes $\left\{D\left(\sigma_{D}\left(\overline{V_{D}\left(n_{0}\right)}\right)\right) \mid D \in G_{C_{\lambda}} \cdot \mathcal{D}_{\lambda}\right\}$. Namely, put

$$
W_{D}(n)=\left(\overline{V_{D}^{-1 / n}}\right)^{\circ} .
$$

Lemma 4.15 (iv) implies $U_{D}\left(\sigma_{D}\left(\overline{V_{D}(n)}\right)=V_{D}(n)\right.$. Thus we get open subsets $W_{D}(n) \subseteq$ $V_{D}$ and a natural number $N^{\prime}$ such that for every choice of natural numbers $\left\{n_{D} \mid D \in\right.$ $\left.G_{C_{\lambda}} \cdot \mathcal{D}_{\lambda}\right\}$ satisfying $n_{D} \geq N^{\prime}$ and $n_{g D}=n_{D}$ for $g \in G_{C_{\lambda}}$ and $D \in G_{C_{\lambda}} \cdot \mathcal{D}_{\lambda}$ the collection $\left\{W_{D}\left(n_{D}\right) \mid D \in G_{C_{\lambda}} \cdot \mathcal{D}_{\lambda}\right\}$ has the following properties:

- $W_{D}\left(n_{D}\right)$ is a $G_{D}$-invariant subset of the $G_{D}$-space $V_{D}$;

- $W_{D}\left(n_{D}\right)=\left(\overline{W_{D}\left(n_{D}\right)}\right)^{\circ}$;

- We have $W_{D}\left(n_{D}\right) \subseteq \overline{W_{D}\left(n_{D}\right)} \subseteq V_{D}$ for $D \in G_{C_{\lambda}} \cdot \mathcal{D}_{\lambda}$;

- $W_{g D}\left(n_{g D}\right)=g W_{D}\left(n_{D}\right)$ holds for $g \in G_{\lambda}$ and $D \in G_{C_{\lambda}} \cdot \mathcal{D}_{\lambda}$;

- $K_{\Xi} \subseteq \bigcup_{\substack{D \notin G \in \mathcal{D} \cdot \mathcal{D}_{\lambda} \\(-\epsilon, \epsilon)}}\left(D^{\circ}\right) \cup \bigcup_{g \in G} \bigcup_{D \in \mathcal{D}_{\lambda}} g \cdot \Phi_{(-\epsilon, \epsilon)}\left(D\left(\sigma_{D}\left(\overline{W_{D}\left(n_{D}\right)}\right)\right)^{\circ}\right)$. 
Consider $D, E \in \mathcal{C}$ with $V_{D} \neq V_{E}$. Since $V_{D}=\bigcup_{n \geq 1} W_{D}(n)$ and $V_{E}=\bigcup_{n \geq 1} W_{E}(n)$ holds, we can find $N^{\prime}(D, E)$ such that $V_{D} \cap V_{E}$ does not contain both $W_{D}(n)$ and $W_{E}(n)$ for $n \geq N^{\prime}(D, E)$. Define $N^{\prime \prime}$ to be the maximum over the numbers $N^{\prime}(D, E)$ for $D, E \in G_{C_{\lambda}} \cdot \mathcal{D}_{\lambda}$ with $V_{D} \neq V_{E}$ and $N^{\prime}$. Put $W_{D}=W_{D}\left(N^{\prime \prime}\right)$ for $D \in G_{C_{\lambda}} \cdot \mathcal{D}_{\lambda}$. Then the collections $\left\{V_{D} \mid D \in G_{C_{\lambda}} \cdot \mathcal{D}_{\lambda}\right\}$ and $\left\{W_{D} \mid D \in G_{C_{\lambda}} \cdot \mathcal{D}_{\lambda}\right\}$ have all the desired properties. This finishes the proof of Lemma 4.20.

Now we can prove Lemma 4.17.

Proof In the sequel we will use the collections $\left\{V_{D} \mid D \in G_{C_{\lambda}} \cdot \mathcal{D}_{\lambda}\right\}$ and $\left\{W_{D} \mid D \in\right.$ $\left.G_{C_{\lambda}} \cdot \mathcal{D}_{\lambda}\right\}$ appearing in Lemma 4.20. We apply Proposition 3.2 in the case, where the space $Z$ is $S_{C_{\lambda}}$, the finite group $F$ is $G_{C_{\lambda}}$, the collection $\mathcal{U}$ is $\left\{V_{D} \mid D \in G_{C_{\lambda}} \cdot \mathcal{D}_{\lambda}\right\}$ and we use the subsets $W_{D} \subseteq V_{D}$. Since $S_{C_{\lambda}} \subseteq X$ is closed, we have $\operatorname{dim}\left(S_{C_{\lambda}}\right) \leq d_{X}$. So from Proposition 3.2 we obtain for every $D \in G_{C_{\lambda}} \cdot \mathcal{D}_{\lambda}$ an open subset $V_{D}^{\prime \prime} \subseteq S_{C_{\lambda}}$ such that the following holds:

- $W_{D} \subseteq V_{D}^{\prime \prime} \subseteq V_{D}$;

- If $\mathcal{U}_{0} \subseteq\left\{V_{D}^{\prime \prime} \mid D \in G_{C_{\lambda}} \cdot \mathcal{D}_{\lambda}\right\}$ has more than $m=k_{G} \cdot\left(d_{X}+1\right)$ elements, then

$$
\bigcap_{U^{\prime \prime} \in \mathcal{U}_{0}} \partial U^{\prime \prime}=\varnothing
$$

- $\left(V_{g D}\right)^{\prime \prime}=\left(g V_{D}\right)^{\prime \prime}=g\left(V_{D}^{\prime \prime}\right)$ for $g \in G_{C_{\lambda}}$ and $D \in G_{C_{\lambda}} \cdot \mathcal{D}_{\lambda}$.

Now define

$$
V_{D}^{\prime}={\overline{V_{D}^{\prime \prime}}}^{\circ}
$$

Since $V_{D}^{\prime \prime}$ is open, we conclude $V_{D}^{\prime}={\overline{V_{D}^{\prime}}}^{\circ}$. Recall that $V_{D}={\overline{V_{D}}}^{\circ}$ and $W_{D}={\overline{W_{D}}}^{\circ}$. Notice that $V_{D}^{\prime \prime}$ is not necessarily $V_{D}^{\prime}$. Since $V_{D}^{\prime \prime}$ is open, we get $V_{D}^{\prime \prime} \subseteq V_{D}^{\prime}$ and hence $V_{D}^{\prime \prime} \cap \partial V_{D}^{\prime}=\varnothing$. We have

$$
\partial V_{D}^{\prime} \subseteq \overline{V_{D}^{\prime}}=\overline{\overline{V_{D}^{\prime \prime}}}=\overline{V_{D}^{\prime \prime}} .
$$

Hence $\partial V_{D}^{\prime} \subseteq \partial V_{D}^{\prime \prime}$. Thus we have constructed for every $D \in G_{C_{\lambda}} \cdot \mathcal{D}_{\lambda}$ an open subset $V_{D}^{\prime} \subseteq S_{C_{\lambda}}$ such that the following holds

- $W_{D} \subseteq V_{D}^{\prime} \subseteq V_{D}$;

- If $\mathcal{V}_{0} \subseteq\left\{V_{D}^{\prime} \mid D \in G_{C_{\lambda}} \cdot \mathcal{U}\left(\mathcal{D}_{\lambda}\right)\right\}$ has more than $m=k_{G} \cdot\left(d_{X}+1\right)$ elements, then

$$
\bigcap_{V^{\prime} \in \mathcal{U}_{0}} \partial V^{\prime}=\varnothing
$$

- $\left(V_{g D}\right)^{\prime}=\left(g V_{D}\right)^{\prime}=g\left(V_{D}^{\prime}\right)$ for $g \in G_{C_{\lambda}}$ and $D \in G_{C_{\lambda}} \cdot \mathcal{U}\left(\mathcal{D}_{\lambda}\right)$; 
- ${\overline{V_{D}^{\prime}}}^{\circ}=V_{D}^{\prime}$.

We use next restriction of boxes to diminish some of the boxes in $\mathcal{D}$ as follows. Consider $D \in \mathcal{D}$. Suppose there exists $g_{0} \in G$ and $D_{0} \in G_{C_{\lambda}} \cdot \mathcal{D}_{\lambda}$ with $D=g_{0} D_{0}$. Then define $\widehat{D}=g_{0} D_{0}\left(\sigma_{D_{0}}\left(\overline{V_{D_{0}}^{\prime}}\right)\right)$. We have to check that this is well-defined. Suppose we have $g_{i} \in G$ and $D_{i} \in G_{C_{\lambda}} \cdot \mathcal{D}_{\lambda}$ with $D=g_{i} D_{i}$ for $i=0,1$. We have $D_{1}=g_{1}^{-1} g_{0} D_{0}$. This implies $g_{1}^{-1} g_{0} \in G_{C_{\lambda}}$ (see Lemma 4.15 (i)) and hence $g_{1}^{-1} g_{0} U_{D_{0}}=U_{D_{1}}$. We conclude $g_{1}^{-1} g_{0}\left(V_{D_{0}}\right)^{\prime}=\left(V_{D_{1}}\right)^{\prime}$ and hence $g_{1}^{-1} g_{0} D_{0}\left(\sigma_{D_{0}}\left(\overline{V_{D_{0}}^{\prime}}\right)\right)=D_{1}\left(\sigma_{D_{1}}\left(\overline{V_{D_{1}}^{\prime}}\right)\right)$. If there does not exist $g_{0} \in G$ and $D_{0} \in \mathcal{D}_{\lambda}$ with $D=g_{0} D_{0}$, we put $\hat{D}=D$. Define a new collection of boxes

$$
\widehat{\mathcal{D}}=\{\widehat{D} \mid D \in \mathcal{D}\} .
$$

Next we want to show that $\hat{\mathcal{D}}$ is a $\delta_{r}$-good box cover of $K_{\Xi}=\bigcup_{\xi \in \Xi} A_{\xi}$. Since $W_{D} \subseteq V_{D}^{\prime}$ for $D \in \mathcal{D}_{\lambda}$, we conclude from property (v) appearing in Lemma 4.20

$$
K_{\Xi} \subseteq \bigcup_{\widehat{D} \in \hat{\mathcal{D}}} \Phi_{(-\epsilon, \epsilon)}\left(\hat{D}^{\circ}\right)
$$

One easily checks that the other required properties of a $\delta_{r}$-good box cover do pass from $\mathcal{D}$ to $\widehat{\mathcal{D}}$ since elements in $\widehat{\mathcal{D}}$ are obtained from those in $\mathcal{D}$ by restriction in a $G$-equivariant way.

We conclude $G_{C_{\lambda}} \cdot \mathcal{U}\left(\widehat{\mathcal{D}}_{\lambda}\right) \subseteq\left\{V_{D}^{\prime} \mid D \in G_{C_{\lambda}} \cdot \mathcal{D}_{\lambda}\right\}$ from Lemma 4.15 (iv). (We remark that $D \cap B_{\lambda} \neq \varnothing$ does not necessarily imply $\widehat{D} \cap B_{\lambda} \neq \varnothing$ and the above inclusion may be a strict inclusion.) By construction $\hat{\mathcal{D}}$ satisfies (4.18).

Suppose for $\hat{D}, \hat{D}^{\prime} \in G_{C_{\lambda}} \cdot \widehat{\mathcal{D}}_{\lambda}$ that $U_{\widehat{D}}=U_{\hat{D}^{\prime}}$. By construction $U_{\widehat{D}}=V_{D}^{\prime}$ and $U_{\widehat{D}}=V_{D^{\prime}}^{\prime}$. We conclude $V_{D}^{\prime}=V_{D^{\prime}}^{\prime}$ and hence both $W_{D}$ and $W_{D^{\prime}}$ are contained in $V_{D} \cap V_{D^{\prime}}$. Properties (vi) and (vii) appearing in Lemma 4.20 imply $D=D^{\prime}$. We conclude that $\hat{\mathcal{D}}$ satisfies (4.19). This finishes the proof of Lemma 4.17.

Now we have finished our arrangement of $\mathcal{D}$ and can now construct the desired new collection $\mathcal{D}^{\prime}$ out of $\mathcal{D}$ as demanded in Lemma 4.13.

\subsection{Carrying out the induction step}

Recall that we defined numbers $m, M, a, b$ and $c$ in the beginning of Section 4.1. Recall also that $N$ is the number of $G$-orbits of $\Lambda$ and that $\epsilon$ is given in Proposition 4.1. 
In the sequel we will abbreviate

We have

$$
\begin{aligned}
& a_{ \pm}= \pm l_{A_{\lambda}} / 2 ; \\
& b_{ \pm}= \pm l_{B_{\lambda}} / 2 ; \\
& c_{ \pm}= \pm l_{C_{\lambda}} / 2 .
\end{aligned}
$$

$$
\begin{aligned}
& a_{+}-a_{-}=a ; \\
& b_{+}-a_{+}=a_{-}-b_{-}=b ; \\
& c_{+}-b_{+}=b_{-}-c_{-}=c .
\end{aligned}
$$

Put

$$
\mu:=\frac{\epsilon}{(N+1)(m+1)} \quad \text { and } \quad \eta:=\frac{\mu}{5} .
$$

We will use the $G_{C_{\lambda}}$-homeomorphism

$$
\mu_{C_{\lambda}}: S_{C_{\lambda}} \times\left[c_{-}, c_{+}\right] \rightarrow C_{\lambda}, \quad(x, \tau) \mapsto \Phi_{\tau}(x)
$$

from Lemma 2.6 (iii) as an identification. Note that $A_{\lambda}=S_{A_{\lambda}} \times\left[a_{-}, a_{+}\right], B_{\lambda}=$ $S_{B_{\lambda}} \times\left[b_{-}, b_{+}\right]$and $B_{\lambda}^{\circ}=\left(S_{B_{\lambda}} \cap B^{\circ}\right) \times\left(b_{-}, b_{+}\right)$under this identification. Note that for $g \in G_{C_{\lambda}}$ we have $g \cdot(x, t)=(g x, t)$, by Lemma 2.6 (iii).

Choose a $G_{C_{\lambda}}$-invariant metric $d_{S_{C_{\lambda}}}$ on $S_{C_{\lambda}}$. Consider $D \in \mathcal{D}_{\lambda}$. Recall that $D \subseteq$ $C_{\lambda}$ and that the retraction $\pi_{C_{\lambda}}: C_{\lambda} \rightarrow S_{C_{\lambda}}$ induces a $G_{D}$-homeomorphism $S_{D} \rightarrow$ $\pi_{C_{\lambda}}\left(S_{D}\right)$. We have introduced the continuous $G_{D}$-invariant map

$$
\tau_{D}: \pi_{C_{\lambda}}\left(S_{D}\right) \rightarrow\left[c_{-}, c_{+}\right]
$$

in Definition 4.14. It is uniquely characterized by $\mu_{C_{\lambda}}(x)=\left(\pi_{C_{\lambda}}(x), \tau_{D} \circ \pi_{C_{\lambda}}(x)\right)$ for $x \in S_{D}$. Since $\tau_{D}$ is continuous and $\pi_{C_{\lambda}}\left(S_{D}\right)$ is compact, we can find $\delta_{D}>0$ such that

$$
\left|\tau_{D}(x)-\tau_{D}(y)\right| \leq \eta \quad \text { for } x, y \in \pi_{C_{\lambda}}\left(S_{D}\right) \text { with } d_{S_{C_{\lambda}}}(x, y)<\delta_{D} .
$$

Because $\mathcal{D}_{\lambda}$ is finite we can set

$$
\delta:=\min \left\{\delta_{D} \mid D \in \mathcal{D}_{\lambda}\right\} .
$$

Then $\delta>0$.

In the sequel interiors and closures of subsets of $S_{C_{\lambda}}$ are to be understood with respect to $S_{C_{\lambda}}$. One easily checks with this convention that Lemma 2.6 (iii) implies $S_{B_{\lambda}}^{\circ}=B^{\circ} \cap S_{B_{\lambda}}$ since $B_{\lambda} \subseteq C_{\lambda}^{\circ}$.

Next we want to apply Proposition 3.3 to the locally connected compact metric space $Z:=S_{C_{\lambda}}$ with the obvious isometric $F:=G_{B_{\lambda}}$-action (note that $F \subseteq G_{C_{\lambda}}$ by 
Lemma 4.15 (i)), the $F=G_{B_{\lambda}}$-invariant open subset $Y:=S_{C_{\lambda}} \cap C_{\lambda}^{\circ}$ which is locally connected by Lemma 2.6 (iv), the collection of sets $\mathcal{U}:=\mathcal{U}\left(\mathcal{D}_{\lambda}\right)$ and the number $\delta>0$ in (4.28). Note that for $D \in \mathcal{D}_{\lambda}$ we have by definition $D \cap B_{\lambda} \neq \varnothing$ and therefore $D \subseteq C_{\lambda}^{\circ}$ since $D$ is not huge. Therefore $\overline{U_{D}} \subseteq \pi_{C_{\lambda}}(D) \subseteq S_{C_{\lambda}} \cap C_{\lambda}^{\circ}=Y$ for $D \in \mathcal{D}_{\lambda}$. In the notation just introduced this means $\bar{U} \subset Y$ for $U \in \mathcal{U}$. Thus we can indeed apply Proposition 3.3 in this situation. By intersecting the resulting open covering with $S_{B_{\lambda}}^{\circ}=S_{B_{\lambda}} \cap B^{\circ}$, we obtain a collection $\mathcal{V}=\mathcal{V}^{0} \cup \mathcal{V}^{1} \cup \cdots \cup \mathcal{V}^{m}$ of open subsets of $S_{B_{\lambda}}^{\circ}$ which has the following properties:

(4.29) $\mathcal{V}$ is a open covering of $S_{B_{\lambda}}^{\circ}$ consisting of finitely many elements;

(4.30) For every $V \in \mathcal{V}$ there are at most $k_{G}^{2} \cdot\left(d_{X}+1\right)$ different $U \in \mathcal{U}\left(\mathcal{D}_{\lambda}\right)$ such that $U \cap V \neq \varnothing$ and $V \not \subset U$;

(4.31) For fixed $j$ and $V_{0} \in \mathcal{V}^{j}$ we have $V_{0} \cap V \neq \varnothing$ for at most $2^{j+1}-2<2^{m+1}$ different subsets $V \in \mathcal{V}^{0} \cup \cdots \cup \mathcal{V}^{j-1}$;

(4.32) For fixed $j$ and $V_{0}, V_{1} \in \mathcal{V}^{j}$ we have either $V_{0}=V_{1}$ or $\overline{V_{0}} \cap \overline{V_{1}}=\varnothing$;

(4.33) Each $\mathcal{V}^{i}$ is $G_{B_{\lambda}}$-invariant, ie, $g V \in \mathcal{V}^{i}$ if $g \in G_{B_{\lambda}}, V \in \mathcal{V}^{i}$;

(4.34) For $V \in \mathcal{V}$ its closure $\bar{V}$ is a $\mathcal{F}$ in-subset of $S_{B_{\lambda}}$ with respect to the $G_{B_{\lambda}-}$ action;

(4.35) We have $\bar{V}^{\circ}=V$ for $V \in \mathcal{V}$;

(4.36) For every $V \in \mathcal{V}$ the diameter of $V$ is bounded by $\delta$;

(4.37) $\mathcal{V}^{j} \cap \mathcal{V}^{k}=\varnothing$ if $j \neq k$.

Properties (4.29), (4.30), (4.31), (4.32), (4.33) and (4.36) are direct consequences of Proposition 3.3. Property (4.34) follows from properties (4.32) and (4.33). Property (4.37) can be achieved by replacing $\mathcal{V}^{j}$ by a subset of $\mathcal{V}^{j}$ if necessary.

Since for every subset $Y \subseteq S_{C_{\lambda}}$ with $\bar{Y}^{\circ}=Y$ we have

$$
\left(\overline{Y \cap S_{B_{\lambda}}^{\circ}}\right)^{\circ} \subseteq\left(\bar{Y} \cap S_{B_{\lambda}}\right)^{\circ}=\bar{Y}^{\circ} \cap S_{B_{\lambda}}^{\circ}=Y \cap S_{B_{\lambda}}^{\circ} \subseteq\left(\overline{Y \cap S_{B_{\lambda}}^{\circ}}\right)^{\circ}
$$

and hence $\left(\overline{Y \cap S_{B_{\lambda}}^{\circ}}\right)^{\circ}=Y \cap S_{B_{\lambda}}^{\circ}$, property (4.35) holds. We mention that because of (4.34) we can consider for $V \in \mathcal{V}$ the restriction $B_{\lambda}(\bar{V})$ and property (4.35) ensures

$$
\begin{aligned}
& S_{B_{\lambda}(\bar{V})}=\bar{V} ; \\
& S_{B_{\lambda}(\bar{V})}^{\circ}=S_{B_{\lambda}(\bar{V})} \cap B_{\lambda}^{\circ}=V .
\end{aligned}
$$

The collection $\mathcal{D}^{\prime}$ we are seeking will be of the form $\mathcal{D} \cup\left\{g B_{\lambda}\left(\bar{W} ; a_{-}^{W}, a_{+}^{W}\right) \mid W \in\right.$ $\mathcal{W}, g \in G\}$, where $\mathcal{W} \subseteq \mathcal{V}$. In order to find suitable $\mathcal{W} \subseteq \mathcal{V}$ and $a_{ \pm}^{W}$ we proceed by a 
subinduction over $n=-1, \ldots, m$. Using (4.12) and (4.25) we set

$$
\delta_{r, n}:=\delta_{r}-(n+1) \cdot \mu \text { for } n=-1,0,1, \ldots, m .
$$

Clearly $\quad \delta_{r}=\delta_{r,-1}>\delta_{r, 0}>\cdots>\delta_{r, n-1}>\delta_{r, n}>\cdots>\delta_{r, m}=\delta_{r+1}$

and $\delta_{r, n-1}-\delta_{r, n}=\mu$. For $j=0, \ldots, m$ let

$$
K_{\lambda}^{(j)}:=\bigcup_{V \in \mathcal{V}_{0} \cup \cdots \cup \mathcal{V}_{j}} V \times\left[a_{-}, a_{+}\right]
$$

(Recall that we use (4.26) to identify $K_{\lambda}^{(j)}$ with a subset of $C_{\lambda}$.) The induction step from $(n-1)$ to $n$ is formulated in the following lemma.

Lemma 4.38 (Induction step : $n-1$ to $n$ ) Assume that we have for $j=0, \ldots, n-1$ subsets $\mathcal{W}^{j} \subseteq \mathcal{V}^{j}$ and numbers $\left\{a_{+}^{W} \mid W \in \mathcal{W}^{j}\right\}$ satisfying $b_{-} \leq a_{-}^{W}<a_{+}^{W} \leq b_{+}$ such that the collection of boxes $\mathcal{D}^{n-1}=\mathcal{D} \cup\left\{g D^{W} \mid W \in \mathcal{W}^{0} \cup \cdots \cup \mathcal{W}^{n-1}, g \in G\right\}$ is a $\delta_{r, n-1}-$ good box cover of $K_{\Xi} \cup G K_{\lambda}^{(n-1)}$, where $D^{W}:=B_{\lambda}\left(\bar{W} ; a_{-}^{W}, a_{+}^{W}\right)$ for $W \in \mathcal{W}^{0} \cup \cdots \cup \mathcal{W}^{n-1}$.

Then there is a subset $\mathcal{W}^{n} \subseteq \mathcal{V}^{n}$ and numbers $a_{ \pm}^{W} \in \mathbb{R}$ with $b_{-} \leq a_{-}^{W}<a_{+}^{W} \leq b_{+}$for $W \in \mathcal{W}^{n}$ such that $\mathcal{D}^{n}=\mathcal{D}^{n-1} \cup\left\{g D^{W} \mid W \in \mathcal{W}^{n}, g \in G\right\}$ is a $\delta_{r, n}$-good box cover of $K_{\Xi} \cup G K_{\lambda}^{(n)}$, where $D^{W}=B_{\lambda}\left(\bar{W} ; a_{-}^{W}, a_{+}^{W}\right)$ for $W \in \mathcal{W}^{n}$.

Since (4.29) implies $S_{A_{\lambda}} \subseteq S_{B_{\lambda}}^{\circ}=\bigcup_{V \in \mathcal{V}} V$ we get $A_{\lambda} \subseteq \bigcup_{V \in \mathcal{V}} V \times\left[a_{-}, a_{+}\right]$. We conclude $K_{\Xi^{\prime}} \subseteq K_{\Xi} \cup G K_{\lambda}^{(m)}$. ( $K_{\Xi^{\prime}}$ was defined in Lemma 4.13.) Hence $\mathcal{D}^{\prime}=\mathcal{D}^{m}$ is the desired $\delta_{r+1}$-good box cover of $K_{\Xi^{\prime}}$. Therefore Lemma 4.13 follows from Lemma 4.38 .

The proof of Lemma 4.38 will occupy the remainder of Section 4.

Definition 4.39 Let $\mathcal{W}^{n}$ be the set of all $W \in \mathcal{V}^{n}$ for which

$$
W \times\left[a_{-}, a_{+}\right] \not \subset \bigcup_{D \in \mathcal{D}^{n-1}} \Phi_{(-\epsilon, \epsilon)}\left(D^{\circ}\right) .
$$

Lemma 4.40 Let $V \in \mathcal{V}$ and let $\partial_{ \pm} D$ be the top or bottom of a box $D \in \mathcal{D}_{\lambda}$. Consider $t \in\left(c_{-}, c_{+}\right)$. Suppose that $\partial_{ \pm} D \cap(V \times\{t\}) \neq \varnothing$. Then

$$
\partial_{ \pm} D \cap\left(V \times\left[c_{-}, c_{+}\right]\right) \subseteq V \times(t-\eta, t+\eta) .
$$

Proof Consider $v \in V$ with $(v, t) \in \partial_{ \pm} D$. Then $\left(v, t \mp l_{D} / 2\right) \in S_{D}$. Hence $\tau_{D}(v)=$ $t \mp l_{D} / 2$, where $\tau_{D}$ is the function introduced in Definition 4.14. Consider $w \in V$ and $s \in\left[c_{-}, c_{+}\right]$with $(w, s) \in \partial_{ \pm} D$. Then $s \mp l_{D} / 2=\tau_{D}(w)$. From (4.27), (4.28) 
and (4.36) we conclude $\left|\tau_{D}(v)-\tau_{D}(w)\right|<\eta$ and hence $|t-s|<\eta$. This implies $\partial_{ \pm} D \cap\left(V \times\left[c_{-}, c_{+}\right]\right) \subseteq V \times(t-\eta, t+\eta)$.

Recall that for $D \in \mathcal{D}_{\lambda}$ we have $D \subseteq C_{\lambda}^{\circ}$ and we have associated to such $D$ an open subset $U_{D}=\pi_{C_{\lambda}}\left(S_{D} \cap D^{\circ}\right)=\pi_{C_{\lambda}}\left(D^{\circ}\right)$ of $S_{C_{\lambda}}$.

Definition 4.41 For $W \in \mathcal{W}^{n}$ define

$$
\begin{aligned}
& \mathcal{D}_{W}:=\left\{D \in \mathcal{D}^{n-1} \mid D^{\circ} \cap W \times\left(b_{-}, b_{+}\right) \neq \varnothing\right\} ; \\
& \mathcal{D}_{W}^{\text {good }}:=\left\{D \in \mathcal{D}_{W} \mid W \subseteq U_{D}\right\} \text {; } \\
& \mathcal{D}_{W}^{\mathrm{bad}}:=\left\{D \in \mathcal{D}_{W} \mid W \cap U_{D} \neq \varnothing, W \nsubseteq U_{D}\right\} ; \\
& J_{W}^{\text {good, } \pm}:=\left\{t \in\left(b_{-}, b_{+}\right)\left|\exists D \in \mathcal{D}_{W}^{\text {good }}\right| \partial_{ \pm} D^{\circ} \cap W \times\{t\} \neq \varnothing\right\} ; \\
& J_{W}^{\mathrm{bad}, \pm}:=\left\{t \in\left(b_{-}, b_{+}\right)\left|\exists D \in \mathcal{D}_{W}^{\mathrm{bad}}\right| \partial_{ \pm} D^{\circ} \cap W \times\{t\} \neq \varnothing\right\} ; \\
& J_{W}^{\text {good }}:=\left\{t \in\left(b_{-}, b_{+}\right)\left|\exists D \in \mathcal{D}_{W}^{\text {good }}\right| D^{\circ} \cap W \times\{t\} \neq \varnothing\right\} ; \\
& J_{W}^{\partial}:=J_{W}^{\mathrm{good},-} \cup J_{W}^{\mathrm{good},+} \cup J_{W}^{\mathrm{bad},-} \cup J_{W}^{\mathrm{bad},+} .
\end{aligned}
$$

Since $D \in \mathcal{D}_{W}$ implies $W \cap U_{D} \neq \varnothing$, we have $\mathcal{D}_{W}=\mathcal{D}_{W}^{\text {good }} \cup \mathcal{D}_{W}^{\text {bad }}$. The reason for the names of $\mathcal{D}_{W}^{\text {good }}$ and $\mathcal{D}_{W}^{\text {bad }}$ is this. In the construction of $D^{W}$ for $W \in \mathcal{W}^{n}$ we will be able to allow top and bottom of $D^{W}$ to be very close to top or bottom of a box in $\mathcal{D}_{W}^{\text {good }}$ (compare Lemma 4.43) but will have to make sure that top and bottom of $D^{W}$ will be far away from top and bottom of every box in $\mathcal{D}_{W}^{\text {bad }}$. Thus, both for choosing $a_{-}^{W}$ and $a_{+}^{W}$ there will be two cases: either we find a suitable top (for $a_{-}^{W}$ ) respectively bottom (for $a_{+}^{W}$ ) of a box in $\mathcal{D}_{W}^{\text {good }}$ to put $W \times\left\{a_{\mp}^{W}\right\}$ close by, or all boxes from $\mathcal{D}_{W}^{\text {good }}$ are far away and we will only have to worry about the boxes from $\mathcal{D}_{W}^{\text {bad }}$. The crucial point will then be, that the number of members of $\mathcal{D}_{W}^{\text {bad }}$ is uniformly bounded, see Lemma 4.42 .

Note that for $g \in G_{B_{\lambda}}$ we have $J_{W}^{\mathrm{good}, \pm}=J_{g W}^{\mathrm{good}, \pm}, J_{W}^{\mathrm{bad}, \pm}=J_{g W}^{\mathrm{bad}, \pm}, J_{W}^{\mathrm{good}}=J_{g W}^{\mathrm{good}}$ and $J_{W}^{\partial}=J_{g W}^{\partial}$, because $g$ acts trivially on the second factor of $C_{\lambda}=S_{C_{\lambda}} \times\left[c_{-}, c_{+}\right]$.

Lemma 4.42 We have

$$
\left|\mathcal{D}_{W}^{\mathrm{bad}}\right| \leq M=\left(k_{G}\right)^{2} \cdot\left(d_{X}+1\right)+2^{m+1}
$$

Proof We conclude from the definitions, Lemma 4.15 (iv) and (4.35)

$$
\begin{aligned}
\mathcal{D}^{n-1} & =\mathcal{D} \cup\left\{g D^{W} \mid W \in \mathcal{W}^{0} \cup \cdots \cup \mathcal{W}^{n-1}, g \in G\right\} ; \\
D^{W} & =B_{\lambda}\left(\bar{W} ; a_{-}^{W}, a_{+}^{W}\right)=\bar{W} \times\left[a_{-}^{W}, a_{+}^{W}\right] \quad \text { for } W \in \mathcal{W}^{0} \cup \cdots \cup \mathcal{W}^{n}, \\
U_{D^{W}} & =W \quad \text { for } W \in \mathcal{W}^{0} \cup \cdots \cup \mathcal{W}^{n} .
\end{aligned}
$$


Recall that $g B_{\lambda} \cap B_{\lambda} \neq \varnothing \Rightarrow g \in G_{B_{\lambda}}$ holds. Hence we get

$$
\begin{aligned}
& \mathcal{D}_{W}^{\mathrm{bad}}=\left\{D \in \mathcal{D} \mid D^{\circ} \cap W \times\left(b_{-}, b_{+}\right) \neq \varnothing, W \cap U_{D} \neq \varnothing, W \not \subset U_{D}\right\} \\
& \cup\left\{g D^{W^{\prime}} \mid W^{\prime} \in \mathcal{W}^{0} \cup \cdots \cup \mathcal{W}^{n-1}, g \in G,\right. \\
&\left.\left(g D^{W^{\prime}}\right)^{\circ} \cap W \times\left(b_{-}, b_{+}\right) \neq \varnothing, W \cap U_{g D^{W^{\prime}}} \neq \varnothing, W \not \subset U_{g D^{W^{\prime}}}\right\} \\
&=\left\{D \in \mathcal{D} \mid D^{\circ} \cap W \times\left(b_{-}, b_{+}\right) \neq \varnothing, W \cap U_{D} \neq \varnothing, W \not \subset U_{D}\right\} \\
& \cup\left\{g D^{W^{\prime}} \mid W^{\prime} \in \mathcal{W}^{0} \cup \cdots \cup \mathcal{W}^{n-1}, g \in G_{B_{\lambda}}, W \cap g W^{\prime} \neq \varnothing, W \not \subset g W^{\prime}\right\} \\
& \subseteq\left\{D \in \mathcal{D}_{\lambda} \mid W \cap U_{D} \neq \varnothing, W \not \subset U_{D}\right\} \\
& \cup\left\{g D^{W^{\prime}} \mid W^{\prime} \in \mathcal{W}^{0} \cup \cdots \cup \mathcal{W}^{n-1}, g \in G_{B_{\lambda}}, W \cap g W^{\prime} \neq \varnothing, W \not \subset g W^{\prime}\right\} .
\end{aligned}
$$

We have $g D^{W^{\prime}}=D^{g W^{\prime}}$ and $D^{W^{\prime}}=D^{W^{\prime \prime}} \Leftrightarrow W^{\prime}=W^{\prime \prime}$. Hence we conclude using (4.19), (4.30), (4.31) and (4.37) that

$$
\begin{aligned}
\left|\mathcal{D}_{W}^{\text {bad }}\right| & \leq\left|\left\{D \in \mathcal{D}_{\lambda} \mid W \cap U_{D} \neq \varnothing, W \not \subset U_{D}\right\}\right| \\
& +\left|\left\{g D^{W^{\prime}} \mid W^{\prime} \in \mathcal{W}^{0} \cup \cdots \cup \mathcal{W}^{n-1}, g \in G_{B_{\lambda}}, W \cap g W^{\prime} \neq \varnothing, W \not \subset g W^{\prime}\right\}\right| \\
& =\left|\left\{U_{D} \in \mathcal{U}\left(\mathcal{D}_{\lambda}\right) \mid W \cap U_{D} \neq \varnothing, W \not \subset U_{D}\right\}\right| \\
& +\left|\left\{g W^{\prime} \mid W^{\prime} \in \mathcal{W}^{0} \cup \cdots \cup \mathcal{W}^{n-1}, g \in G_{B_{\lambda}}, W \cap g W^{\prime} \neq \varnothing, W \not \subset g W^{\prime}\right\}\right| \\
& \leq\left|\left\{U_{D} \in \mathcal{U}\left(\mathcal{D}_{\lambda}\right) \mid W \cap U_{D} \neq \varnothing, W \not \subset U_{D}\right\}\right| \\
& +\left|\left\{V \mid V \in \mathcal{V}^{0} \cup \cdots \cup \mathcal{V}^{n-1}, W \cap V \neq \varnothing, W \not \subset V\right\}\right| \\
& \leq k_{G}^{2} \cdot\left(d_{X}+1\right)+2^{m+1} \\
& =M .
\end{aligned}
$$

This finishes the proof of Lemma 4.42 .

Lemma 4.43 Let $W \in \mathcal{W}^{n}$. If $t_{0} \in J_{W}^{\text {good, }-} \cup J_{W}^{\text {good, }+}$ and $t_{1} \in J_{W}^{\partial}$, then

$$
\left|t_{0}-t_{1}\right| \notin\left[\epsilon-\delta_{r, n-1}+\eta, \alpha+\delta_{r, n-1}-\eta\right] .
$$

Proof There are $D_{0} \in \mathcal{D}_{W}^{\text {good }}, D_{1} \in \mathcal{D}_{W}, \sigma_{0}, \sigma_{1} \in\{-,+\}$ and $w_{0}, w_{1} \in W$ such that $\left(w_{0}, t_{0}\right) \in \partial_{\sigma_{0}} D_{0}^{\circ}$ and $\left(w_{1}, t_{1}\right) \in \partial_{\sigma_{1}} D_{1}^{\circ}$. By definition of $\mathcal{D}_{W}^{\text {good }}$ we have $W \subseteq U_{D_{0}}$ and $D_{0} \subseteq C_{\lambda}^{\circ}$. Therefore there is $\tau \in \mathbb{R}$ such that $\Phi_{\tau}\left(w_{1}, t_{1}\right)=\left(w_{1}, t_{1}+\tau\right) \in \partial_{\sigma_{0}} D_{0}^{\circ}$ and $t_{1}+\tau \in\left(c_{-}, c_{+}\right)$. We conclude from Lemma 4.40 that $\left|t_{0}-\left(t_{1}+\tau\right)\right|<\eta$. Because 
$\mathcal{D}^{n-1}$ satisfies the induction assumption, we know that $\mathcal{D}_{W} \subseteq \mathcal{D}^{n-1}$ is $\delta_{r, n-1}$-overlong. Therefore

$$
|\tau| \notin\left[\epsilon-\delta_{r, n-1}, \alpha+\delta_{r, n-1}\right] .
$$

This implies our result, because ||$t_{0}-t_{1}|-| \tau|| \leq\left|\left(t_{0}-t_{1}\right)-\tau\right|<\eta$.

Definition 4.44 For $W \in \mathcal{W}^{n}$ let

$$
\begin{aligned}
& R_{-}^{W}:=\sup \left(\left(b_{-}+\alpha+\epsilon, a_{-}\right) \cap J_{W}^{\text {good, }+}\right) \cup\left\{b_{-}+\alpha+\epsilon\right\} \\
& R_{+}^{W}:=\inf \left(\left(a_{+}, b_{+}-\alpha-\epsilon\right) \cap J_{W}^{\text {good, }-}\right) \cup\left\{b_{+}-\alpha-\epsilon\right\}
\end{aligned}
$$

Lemma 4.45 Let $W \in \mathcal{W}^{n}$. We have:

(i) $\left(R_{-}^{W}, R_{+}^{W}\right) \cap J_{W}^{\text {good }}=\varnothing$;

(ii) $R_{-}^{W}+\alpha+\delta_{r, n}+2 \eta<R_{+}^{W}$.

Proof We first show that

$$
\left[a_{-}-\epsilon / 2+\eta, a_{+}+\epsilon / 2-\eta\right] \cap J_{W}^{\text {good }}=\varnothing .
$$

We proceed by contradiction. If (4.46) fails then there are $t_{0} \in\left[a_{-}-\epsilon / 2+\eta, a_{+}+\epsilon / 2-\right.$ $\eta], w_{0} \in W$ and a box $D \in \mathcal{D}_{W}^{\text {good }}$ such that $\left(w_{0}, t_{0}\right) \in D^{\circ}$. We have $W \subseteq U_{D}$. For every $w \in W$ there exist unique real numbers $\tau_{ \pm}(w)$ such that $\left(w, \tau_{ \pm}(w)\right) \in \partial_{ \pm} D^{\circ}$. From Lemma 4.40 we conclude

$$
\tau_{ \pm}(w) \in\left(\tau_{ \pm}\left(w_{0}\right)-\eta, \tau_{ \pm}\left(w_{0}\right)+\eta\right) \quad \text { for } w \in W .
$$

We have $\tau_{-}\left(w_{0}\right) \leq t_{0} \leq \tau_{+}\left(w_{0}\right)$. From $a_{+}-a_{-}=\epsilon / 2$ we conclude $t_{0} \leq a_{+}+\epsilon / 2-\eta=$ $a_{-}+\epsilon-\eta$ and $t_{0} \geq a_{-}-\epsilon / 2+\eta=a_{+}-\epsilon+\eta$. We estimate

$$
\begin{aligned}
& \tau_{-}(w)-\epsilon<\tau_{-}\left(w_{0}\right)+\eta-\epsilon \leq t_{0}+\eta-\epsilon \leq a_{-} ; \\
& \tau_{+}(w)+\epsilon>\tau_{+}\left(w_{0}\right)-\eta+\epsilon \geq t_{0}-\eta+\epsilon \geq a_{+} .
\end{aligned}
$$

This implies $W \times\left[a_{-}, a_{+}\right] \subset \Phi_{(-\epsilon, \epsilon)} D^{\circ}$ which contradicts the definition of $\mathcal{W}^{n}$ in Definition 4.39. This proves (4.46).

We give now the proof of (i). Assume that there is $D \in \mathcal{D}_{W}^{\text {good }}, t_{0} \in\left(R_{-}^{W}, R_{+}^{W}\right)$ and $w_{0} \in W$ such that $\left(w_{0}, t_{0}\right) \in D^{\circ}$. Because $\eta=\mu / 5<\epsilon / 5<\epsilon / 2$ we conclude from (4.46) that either $t_{0}<a_{-}$or $t_{0}>a_{+}$. We treat the first case, in the second case there is an analogous argument. There is $\tau_{+} \geq 0$ such that $\left(w_{0}, t_{0}+\tau_{+}\right) \in \partial_{+} D^{\circ}$. If $t_{0}+\tau_{+}>a_{-}$, then $\left(w_{0}, a_{-}\right) \in D^{\circ}$, that is, $a_{-} \in J_{W}^{\text {good }}$. Since this contradicts (4.46) we conclude $t_{0}+\tau_{+} \leq a_{-}$. Clearly $t_{0}+\tau_{+} \in J_{W}^{\text {good, }}+$ and $b_{-}+\alpha+\epsilon \leq R_{-}^{W}<t_{0} \leq t_{0}+\tau_{+}$. But this contradicts the construction of $R_{-}^{W}$ in Definition 4.44. This proves (i). 
Next we prove (ii). First we treat the case $R_{-}^{W}=b_{-}+\alpha+\epsilon$. Since $2 \eta=2 \mu / 5<$ $2 \epsilon / 5<\epsilon, \delta_{r, n}<\epsilon$ and $2 \alpha+3 \epsilon<b=a_{-}-b_{-}$we conclude

$$
R_{-}^{W}+\alpha+\delta_{r, n}+2 \eta=b_{-}+2 \alpha+\epsilon+\delta_{r, n}+2 \eta<b_{-}+2 \alpha+3 \epsilon<b_{-}+b=a_{-} \leq R_{+}^{W} .
$$

The case $R_{+}^{W}=b_{+}-\alpha-\epsilon$ can be treated similarly. Therefore we may assume now $R_{-}^{W} \neq b_{-}+\alpha+\epsilon$ and $R_{+}^{W} \neq b_{+}-\alpha-\epsilon$. From the construction of $R_{+}^{W}$ we conclude then that there are $t_{ \pm} \in J_{W}^{\text {good, } \mp}$, such that $R_{-}^{W}-\eta<t_{-} \leq R_{-}^{W}$ and $R_{+}^{W} \leq t_{+}<R_{+}^{W}+\eta$. Clearly $t_{-} \leq R_{-}^{W} \leq a_{-}<a_{+} \leq R_{+}^{W} \leq t_{+}$. Thus $t_{+}-t_{-}>0$. By Lemma 4.43

$$
t_{+}-t_{-} \notin\left[\epsilon-\delta_{r, n-1}+\eta, \alpha+\delta_{r, n-1}-\eta\right] \text {. }
$$

On the other hand (4.46) implies $t_{-}<a_{-}-\epsilon / 2+\eta$ and $t_{+}>a_{+}+\epsilon / 2-\eta$. Using $a_{+}-a_{-}=\epsilon / 2,2 \eta=2 \mu / 5<2 \epsilon / 5<\epsilon / 2$ and $\delta_{r, n-1}-\eta=\delta_{r, n}+\mu-\eta>\delta_{r, n} \geq 0$ we estimate

$t_{+}-t_{-}>\left(a_{+}+\epsilon / 2-\eta\right)-\left(a_{-}-\epsilon / 2+\eta\right)=3 \epsilon / 2-2 \eta>\epsilon>\epsilon-\delta_{r, n-1}+\eta$.

Therefore $t_{+}-t_{-}>\alpha+\delta_{r, n-1}-\eta=\alpha+\delta_{r, n}+\mu-\eta$. This implies $t_{-}+\alpha+\delta_{r, n}<$ $t_{+}+\eta-\mu$. Using this and $5 \eta=\mu$ we compute

$$
\begin{aligned}
R_{-}^{W}+\alpha+\delta_{r, n}+2 \eta & <t_{-}+\alpha+\delta_{r, n}+3 \eta \\
& <t_{+}-\mu+4 \eta<R_{+}^{W}-\mu+5 \eta=R_{+}^{W} .
\end{aligned}
$$

We can now give the construction of $a_{ \pm}^{W}$ for $W \in \mathcal{W}^{n}$. If $R_{-}^{W}>b_{-}+\alpha+\epsilon$ then we set $a_{-}^{W}:=R_{-}^{W}+\eta$. Otherwise $R_{-}^{W} \stackrel{ \pm}{=} b_{-}+\alpha+\epsilon$ and we will use the fact that we arranged $b=a_{-}-b_{-}$to be very large. It follows from Lemmas 4.42 and 4.45 (i) and $a_{-} \leq R_{+}^{W}$ that $J_{W}^{\partial} \cap\left[R_{-}^{W}, a_{-}\right]$is contained in the union of $2 M$ intervals of length $2 \eta$. Using $\delta_{r, n}+\eta<\delta_{r, n}+\mu=\delta_{r, n-1}<\epsilon$ we estimate

$$
\begin{aligned}
a_{-}-\left(b_{-}+\alpha+\epsilon\right) & =b-(\alpha+\epsilon)=4 M(\alpha+2 \epsilon)+2(\alpha+\epsilon) \\
& >(2 M+1)(2 \alpha+2 \epsilon)>(2 M+1)\left(2 \alpha+2 \eta+2 \delta_{r, n}\right) .
\end{aligned}
$$

If from an interval $I$ of length strictly larger than $L$, we take out $2 M$ or less intervals, each of which has length less than or equal to $l$, then the remaining set contains an interval of length $\tilde{l}:=(L-2 M l) /(2 M+1)$. The center of such an interval, will have distance $\widetilde{l} / 2$ from all points in the $2 M$ intervals and from the boundary of $I$. Therefore we find $a_{-}^{W} \in\left[\left(b_{-}+\alpha+\epsilon\right)+\left(\alpha+\delta_{r, n}\right), a_{-}-\left(\alpha+\delta_{r, n}\right)\right]$ such that

$$
\left|a_{-}^{W}-t\right|>\alpha+\delta_{r, n} \text { for all } t \in J_{W}^{\partial} .
$$

This finishes the construction of $a_{-}^{W}$. To construct $a_{+}^{W}$ we proceed similarly. If $R_{+}^{W}<b_{+}-\alpha-\epsilon$ then we set $a_{+}^{W}:=R_{+}^{W}-\eta$. Otherwise $R_{+}^{W}=b_{+}-\alpha-\epsilon$ and there 
is $a_{+}^{W} \in\left[a_{+}+\left(\alpha+\delta_{r, n}\right),\left(b_{+}-\alpha+\epsilon\right)-\left(\alpha+\delta_{r, n}\right)\right]$ such that

$$
\left|a_{+}^{W}-t\right|>\alpha+\delta_{r, n} \text { for all } t \in J_{W}^{\partial} .
$$

This finishes the construction of $a_{+}^{W}$. We can arrange that $a_{ \pm}^{W}=a_{ \pm}^{g W}$ for $g \in G_{B_{\lambda}}$.

For $W \in \mathcal{W}^{n}$ let now $D^{W}:=B_{\lambda}\left(\bar{W} ; a_{-}^{W}, a_{+}^{W}\right)=\bar{W} \times\left[a_{-}^{W}, a_{+}^{W}\right]$.

Lemma 4.49 Let $W \in \mathcal{W}^{n}$.

(i) $\Phi_{(-\alpha-\epsilon, \alpha+\epsilon)}\left(D^{W}\right) \subset B_{\lambda}$;

(ii) If $x$ lies in the open bottom or open top of $D^{W}$ then

$$
\Phi_{\left[-\alpha-\delta_{r, n},-\epsilon+\delta_{r, n}\right] \cup\left[\epsilon-\delta_{r, n}, \alpha+\delta_{r, n}\right]}(x)
$$

does not intersect the open bottom or top of a box $D \in \mathcal{D}^{n-1}$;

(iii) $l_{D^{W}}=a_{+}^{W}-a_{-}^{W}>\alpha+\delta_{r, n}$;

(iv) $W \times\left[a_{-}, a_{+}\right] \subseteq \Phi_{(-\epsilon, \epsilon)}\left(D^{W}\right)^{\circ}$;

(v) $\left|\left\{D \in \mathcal{D}^{n-1} \mid D^{\circ} \cap\left(D^{W}\right)^{\circ} \neq \varnothing\right\}\right| \leq M$.

Proof (i) By construction $b_{-}+\alpha+\epsilon<a_{-}^{W}<a_{+}^{W}<b_{+}-\alpha-\epsilon$ and (4.34) implies $\bar{W} \subseteq S_{B_{\lambda}}$.

(ii) We consider the open bottom first. Let $w \in W$ and $x=\left(w, a_{-}^{W}\right)$. By (i) $\Phi_{\left[-\alpha-\delta_{r, n},-\epsilon+\delta_{r, n}\right] \cup\left[\epsilon-\delta_{r, n}, \alpha+\delta_{r, n}\right]}(x)$ is contained in $B_{\lambda}$ and can therefore only intersect boxes from $\mathcal{D}_{W}$. The claim follows thus if $\left|a_{-}^{W}-t\right| \notin\left[\epsilon-\delta_{r, n}, \alpha+\delta_{r, n}\right]$ for all $t \in J_{W}^{\partial}$. If $R_{-}^{W}=b_{-}+\alpha+\epsilon$ then (4.47) holds and implies our claim. Otherwise $a_{-}^{W}=R_{-}^{W}+\eta$ and there is $t_{0} \in J_{W}^{\text {good, }+} \cap\left[R_{-}^{W}-\eta, R_{-}^{W}\right]$ by the construction of $R_{-}^{W}$ in Definition 4.44. Now Lemma 4.43 implies our claim since $\delta_{r, n-1}-\delta_{r, n}=\mu>$ $3 \mu / 5=3 \eta$. The open top can be treated completely analogously.

(iii) Clearly $a_{+}^{W}-a_{-}^{W}$ is the length of $D^{W}$. By construction $a_{-}^{W} \leq a_{-}+\eta<a_{+}$ and $a_{+}^{W} \geq a_{+}-\eta>a_{-}$since $a_{+}-a_{-}=a=\epsilon / 2>\mu / 5=\eta$. If $R_{-}^{W}=b_{-}+\alpha+\epsilon$, then by construction $a_{-}^{W} \leq a_{-}-\left(\alpha+\delta_{r, n}\right)$ and our claim follows. Similarly the claim follows if $R_{+}^{W}=b_{+}-\alpha-\epsilon$. Thus we are left with the case $a_{ \pm}^{W}=R_{ \pm}^{W} \mp \eta$ and the claim follows from Lemma 4.45 (ii).

(iv) As noted above the construction of $a_{ \pm}^{W}$ implies that $a_{-}^{W}-\eta \leq a_{-}$and $a_{+}^{W}+\eta \geq a_{+}$. The claim follows therefore from $\eta<\epsilon$.

(v) Because $\left(D^{W}\right)^{\circ} \subset W \times\left(b_{-}, b_{+}\right)$,

$$
\left\{D \in \mathcal{D}^{n-1} \mid D^{\circ} \cap\left(D^{W}\right)^{\circ} \neq \varnothing\right\}=\left\{D \in \mathcal{D}_{W} \mid D^{\circ} \cap\left(D^{W}\right)^{\circ} \neq \varnothing\right\} .
$$


By construction $R_{-}^{W}<a_{-}^{W}<a_{+}^{W}<R_{+}^{W}$. Thus Lemma 4.45 (i) and Lemma 4.42 imply

$$
\left|\left\{D \in \mathcal{D}_{W} \mid D^{\circ} \cap\left(D^{W}\right)^{\circ} \neq \varnothing\right\}\right|=\left|\left\{D \in \mathcal{D}_{W}^{\mathrm{bad}} \mid D^{\circ} \cap\left(D^{W}\right)^{\circ} \neq \varnothing\right\}\right| \leq M .
$$

We now define

$$
\mathcal{D}^{n}:=\mathcal{D}^{n-1} \cup\left\{g D^{W} \mid W \in \mathcal{W}^{n}, g \in G\right\} .
$$

It remains to check that $\mathcal{D}^{n}$ is a $\delta_{r, n}-\operatorname{good}$ box cover of $K_{\Xi} \cup G K_{\lambda}^{(n)}$. Recall the induction hypothesis that $\mathcal{D}^{n-1}$ is a $\delta_{r, n-1}-\operatorname{good}$ box cover of $K_{\Xi} \cup G K_{\lambda}^{(n-1)}$.

We begin with showing that $\mathcal{D}^{n}$ is $\delta_{r, n}$-overlong. So we have to show for every $x \in X$ which lies on the open bottom or open top of a box $D_{1}$ in $\mathcal{D}^{n}$, that the set $\Phi_{\left[-\alpha-\delta_{r, n},-\epsilon+\delta_{r, n}\right] \cup\left[\epsilon-\delta_{r, n}, \alpha+\delta_{r, n}\right]}(x)$ does not intersect the open bottom or the open top of any box $D_{2}$ in $\mathcal{D}^{n}$.

If $D_{1}$ and $D_{2}$ lie in $\mathcal{D}^{n-1}$, this follows from the induction hypothesis.

Suppose that $D_{1} \notin \mathcal{D}^{n-1}$ and $D_{2} \in \mathcal{D}^{n-1}$. Then we can assume without loss of generality that $D_{1}=D^{W}$ for some $W \in \mathcal{W}^{n}$ since $\mathcal{D}^{n-1}$ is $G$-invariant. The claim follows then from Lemma 4.49 (ii).

The case $D_{1} \in \mathcal{D}^{n-1}$ and $D_{2} \notin \mathcal{D}^{n-1}$ is treated analogously.

If $D_{1}=D_{2}$ and $D_{1} \notin \mathcal{D}^{n-1}$, then the claim follows from Lemma 4.49 (i) and (iii).

If $D_{1} \neq D_{2}$ and $D_{1}, D_{2} \notin \mathcal{D}^{n-1}$, the claim follows from (4.32) and Lemma 4.49 (i) since $B_{\lambda}$ is a $\mathcal{F}$ in-subset of $X$. Hence $\mathcal{D}^{n}$ is $\delta_{r, n}$-overlong.

We conclude from Lemma 4.49 (i) and (iii) that $\mathcal{D}^{n}$ satisfies (4.3).

We derive the inclusion $K_{\Xi} \cup G K_{\lambda}^{(n)} \subset \bigcup_{D \in \mathcal{D}^{n}} \Phi_{(-\epsilon, \epsilon)}\left(D^{\circ}\right)$ from Definition 4.39 and Lemma 4.49 (iv). (The set $K_{\lambda}^{(n)}$ was defined before Lemma 4.38.)

By (4.32) the $D^{W}$ are mutually disjoint. Therefore Lemma 4.49 (v) implies that (4.4) holds for $\mathcal{D}^{n}$.

It is clear that (4.5) and (4.6) hold for $\mathcal{D}^{n}$.

Next we prove property (4.7). Because of the induction hypothesis it suffices to prove the assertion for the boxes $g D^{W}$ for $g \in G$ and $W \in \mathcal{W}^{n}$, where $D^{W}=B_{\lambda}\left(\bar{W} ; a_{-}^{W}, a_{+}^{W}\right)$. From Lemma 4.49 (i) we conclude $\Phi_{[-\alpha-\epsilon, \alpha+\epsilon]}\left(D^{W}\right) \subseteq B_{\lambda}(W)$. Since $\bar{W}$ is a $\mathcal{F}$ insubset of $S_{B_{\lambda}}$ with respect to the $G_{B_{\lambda}}$-action by (4.34) and $B$ is a $\mathcal{F}$ in-subset of the $G$-space $X, \Phi_{[-\alpha-\epsilon, \alpha+\epsilon]}\left(D^{W}\right)$ is a $\mathcal{F}$ in-subset of the $G$-space $X$. 
Finally we show that elements in $\mathcal{D}^{(n)}$ are not huge. For $g \in G$ and $W \in \mathcal{W}^{n}$ the box $g D^{W}$ can be obtained by restriction from $B_{g \lambda}$ and is therefore not huge; compare Definition 4.10 and the subsequent comment.

We have shown that $\mathcal{D}^{n}$ is the required $\delta_{r, n}-\operatorname{good}$ box cover of $K_{\Xi} \cup G K_{\lambda}^{(n)}$. This finishes the proof of Lemma 4.38.

As was noted before, Proposition 4.1 follows from Lemma 4.13 which follows from Lemma 4.38. The proof of Proposition 4.1 is therefore now completed.

\section{Construction of long $\mathcal{V} \mathcal{C} y c-$ covers of $X$}

At the end of this section we will give the proof of Theorem 1.4. Throughout this section we will work in the situation of Convention 1.3. In order to construct the long and thin cover of $X$ we need to discuss covers of $X^{\mathbb{R}}$ and $X_{\leq \gamma}-X^{\mathbb{R}}$.

Lemma 5.1 There exists a collection $\mathcal{U}_{X} \mathbb{R}$ of open $\mathcal{F}$ in-subsets of $X$ such that $G \backslash \mathcal{U}_{X^{\mathbb{R}}}$ is finite, $X^{\mathbb{R}} \subset \bigcup_{U \in \mathcal{U}_{X} \mathbb{R}} U$ and $\operatorname{dim}\left(\mathcal{U}_{X^{\mathbb{R}}}\right)<\infty$.

Proof Because the action of $G$ on $X$ is proper there is for $x \in X^{\mathbb{R}}$ an open $\mathcal{F}$ inneighborhood $W_{x}$ of $x$. Because the action of $G$ on $X$ is cocompact and $X^{\mathbb{R}}$ is closed, there is a finite subset $\Lambda \subset X^{\mathbb{R}}$ such that $X^{\mathbb{R}} \subset \bigcup_{g \in G} \bigcup_{\lambda \in \Lambda} g W_{\lambda}$. Let $\mathcal{U}_{X \mathbb{R}}=\left\{g W_{\lambda} \mid g \in G, \lambda \in \Lambda\right\}$. Because the $W_{\lambda}$ are $\mathcal{F}$ in-sets we have $g W_{\lambda} \neq W_{\lambda} \Rightarrow$ $g W_{\lambda} \cap W_{\lambda}=\varnothing$. Therefore $\operatorname{dim}\left(\mathcal{U}_{X^{\mathbb{R}}}\right) \leq|\Lambda|-1$.

Lemma 5.2 Fix $\gamma>0$. Let $\mathcal{L}_{\leq \gamma}$ be the set of orbits $L=\Phi_{\mathbb{R}}(x)$ in $X$ whose $G$ period satisfies $0<\operatorname{per}_{\Phi}^{G}(L) \leq \gamma$. Then there exists a collection $\mathcal{U}_{\gamma}=\left\{U_{L} \mid L \in \mathcal{L}_{\leq \gamma}\right\}$ of open $\mathcal{V C y c - s u b s e t s} U_{L}$ of the $G$-space $X$ such that $L \subseteq U_{L}$ for $L \in \mathcal{L}_{\gamma}$ and $\operatorname{dim} \mathcal{U}=0$.

Proof By assumption we can find finitely many pairwise distinct elements $L_{1}, L_{2}$, $\ldots, L_{r}$ in $\mathcal{L}_{\leq \gamma}$ such that $\mathcal{L}_{\leq \gamma}=G \cdot\left\{L_{1}, L_{2}, \ldots, L_{r}\right\}$. We can arrange that $L_{j}=g \cdot L_{k}$ for some $g \in G$ implies $j=k$. Since the $G_{L_{j}}$-action on $L_{j}$ is proper and cocompact and $L_{j}$ is homeomorphic to $\mathbb{R}$ or $S^{1}$, the group $G_{L_{j}}$ is virtually cyclic. (A group that acts cocompact and properly on $\mathbb{R}$ has two ends and is therefore virtually cyclic [5, Theorem I.8.32(2)].) We can choose compact subsets $K_{j} \subseteq L_{j}$ with $L_{j}=G_{L_{j}} \cdot K_{j}$ and $G \backslash G L_{j}$ closed.

Since $G \backslash X$ is compact and $G \backslash G L_{j} \cap G \backslash G L_{k} \neq \varnothing \Rightarrow j=k$ holds, we can find open subsets $V_{1}^{\prime}, V_{2}^{\prime}, \ldots, V_{r}^{\prime}$ in $G \backslash X$ such that $G \backslash G L_{j} \subseteq V_{j}^{\prime}$ and $V_{j}^{\prime} \cap V_{k}^{\prime} \neq \varnothing \Rightarrow j=k$ 
holds. Let $V_{j}$ be the preimage of $V_{j}^{\prime}$ under the projection $X \rightarrow G \backslash X$. Then $V_{j}$ is a $G$-invariant open neighborhood of $L_{j}$ and $V_{j} \cap V_{k} \neq \varnothing \Rightarrow j=k$.

Fix $j \in\{1,2, \ldots r\}$. Since the $G$-action on $X$ is proper, we can find an open neighborhood $W_{j}^{\prime}$ of $K_{j}$ and a finite subset $S \subseteq G$ such that $W_{j}^{\prime} \cap g W_{j}^{\prime} \neq \varnothing \Rightarrow g \in S$. Let $S_{0} \subseteq S$ be the subset consisting of those elements $g \in S$ for which $K_{j} \cap g K_{j}=\varnothing$. Since $K_{j}$ is compact, we can find for $s \in S_{0}$ an open neighborhood $W_{s}^{\prime \prime}$ of $K_{j}$ such that $W_{s}^{\prime \prime} \cap s W_{s}^{\prime \prime}=\varnothing$. Put $U_{j}^{\prime}:=W_{j}^{\prime} \cap \bigcap_{s \in S_{0}} W_{s}^{\prime \prime}$. Then $U_{j}^{\prime}$ is an open neighborhood of $K_{j}$ such that $U_{j}^{\prime} \cap g U_{j}^{\prime} \neq \varnothing$ implies $K_{j} \cap g K_{j} \neq \varnothing$. Put $U_{j}=G_{L_{j}} \cdot U_{j}^{\prime}$. Then $U_{j}$ is a $G_{L_{j}}$-invariant open subset containing $L_{j}=G_{L_{j}} K_{j}$. Next we prove $g U_{j} \cap U_{j} \neq \varnothing \Rightarrow g \in G_{L_{j}}$. Suppose for $g \in G$ that $g U_{j} \cap U_{j} \neq \varnothing$. Then we can find $g_{0}, g_{1} \in G_{L_{j}}$ such that $g g_{0} U_{j}^{\prime} \cap g_{1} U_{j} \neq \varnothing$. This implies $g_{1}^{-1} g g_{0} K_{j} \cap K_{j} \neq \varnothing$. We conclude $g_{1}^{-1} g g_{0} L_{j} \cap L_{j} \neq \varnothing$ and hence $g_{1}^{-1} g g_{2} L_{j}=L_{j}$. This shows $g_{1}^{-1} g g_{0} \in G_{L_{j}}$ and thus $g \in G_{L_{j}}$. Hence $U_{j}$ is an open $\mathcal{V} \mathcal{C}$ yc-subset of the $G$-space $X$ such that $G_{U_{j}}=G_{L_{j}}$ and $L_{j} \subseteq U_{j}$.

Define for any element $L \in \mathcal{L}_{\leq \gamma}$

$$
U_{L}=g \cdot\left(V_{j} \cap U_{j}\right) \quad \text { for } g \in G \text { with } L=g L_{j} .
$$

This is independent of the choice of $g$ and $j$ and $U_{L}$ is a $\mathcal{V C y c}$-subset of the $G$ space $X$ with $G_{U_{L}}=G_{L}$ since $\left(V_{j} \cap U_{j}\right)$ is a $\mathcal{V} \mathcal{C}$ yc-subset of the $G$-space $X$ with $G_{V_{j} \cap U_{j}}=G_{L_{j}}$. We have by construction

$$
U_{L_{1}} \cap U_{L_{2}} \neq \varnothing \Rightarrow L_{1}=L_{2}
$$

Finally we can give the proof of Theorem 1.4.

Proof Let $\alpha>0$ be given. Choose $\epsilon$ such that $0<\epsilon<\alpha$. Let $M=M\left(k_{G}, d_{X}\right)$ and $\gamma=\gamma(4 \alpha, \epsilon, M)>0$ be as in Proposition 4.1.

Let $\mathcal{U}_{X} \mathbb{R}$ be the collection of open $\mathcal{F}$ in-sets from Lemma 5.1 and $\mathcal{U}_{\gamma}$ be the collection of open $\mathcal{V} \mathcal{C}$ yc-sets from Lemma 5.2. Note that $\operatorname{dim}\left(\mathcal{U}_{X^{\mathbb{R}}} \cup \mathcal{U}_{\gamma}\right)=\operatorname{dim}\left(\mathcal{U}_{X^{\mathbb{R}}}\right)+1$ is finite and does not depend on $\alpha$, but only on an arbitrarily small neighborhood of $X^{\mathbb{R}}$ as a $G$-space.

Put

$$
S=\left\{x \in X \mid \exists U \in \mathcal{U}_{\gamma} \cup \mathcal{U}_{X^{\mathbb{R}}} \text { such that } \Phi_{[-\alpha, \alpha]}(x) \subset U\right\} .
$$

Note that $S$ is $G$-invariant, because $\mathcal{U}_{\gamma}$ and $\mathcal{U}_{X \mathbb{R}}$ are. Consider $x \in S$. Choose $U_{x} \in \mathcal{U}_{\gamma} \cup \mathcal{U}_{X} \mathbb{R}$ with $\Phi_{[-\alpha, \alpha]}(x) \subseteq U$. Since $\{x\} \times[-\alpha, \alpha]$ is compact and contained in $\Phi^{-1}\left(U_{x}\right)$, we can find an open neighborhood $V_{x}$ of $x$ such that $V_{x} \times[-\alpha, \alpha] \subseteq$ $\Phi^{-1}\left(U_{x}\right)$. This implies $V_{x} \subseteq S$. Hence $S$ is an open $G$-invariant subset of $X$ which contains $X_{\leq \gamma}$. 
Let $K$ be the closure of the complement of $S$ in $X$. Since $G \backslash X$ is compact $K \subseteq X$ is a cocompact $G$-invariant subset which does not meet $X_{\leq \gamma}$. Hence we can apply Proposition 4.1 to $K$ with respect to $4 \alpha$ instead of $\alpha$ and $\epsilon$ with $0<\epsilon<\alpha$. Recall that $M=M\left(k_{G}, d_{X}\right)$ and $\gamma=\gamma(M, 4 \alpha, \epsilon)$ are the numbers appearing in Proposition 4.1. So we get a collection of boxes $\mathcal{D}$ with the properties described in Proposition 4.1. Put

$$
\mathcal{U}_{K}=\left\{\Phi_{(-\alpha-\epsilon,+\alpha+\epsilon)} D^{\circ} \mid D \in \mathcal{D}\right\} .
$$

Then for $x \in K$ there is $U \in \mathcal{U}_{K}$ such that $\Phi_{[-\alpha, \alpha]}(x) \subset U$ and every element in $\mathcal{U}_{K}$ is an open $\mathcal{F}$ in-subset of $X$.

Next we show $\operatorname{dim}\left(\mathcal{U}_{K}\right) \leq 2 M+1$. Consider pairwise disjoint elements $D_{1}, D_{2}$, $\ldots, D_{2 M+3}$ of $\mathcal{D}$. We have to show that $\bigcap_{k=1}^{2 M+3} \Phi_{(-\alpha-\epsilon, \alpha+\epsilon)}\left(D_{k}^{\circ}\right)=\varnothing$. Suppose the contrary, ie, there exist $x \in X$ such that $x \in \Phi_{(-\alpha-\epsilon, \alpha+\epsilon)}\left(D_{k}^{\circ}\right)$ holds for $k=$ $1,2, \ldots,(2 M+3)$. Obviously $x \in \Phi_{(-\alpha-\epsilon, \alpha+\epsilon)}\left(D_{k}^{\circ}\right)$ implies that $\Phi_{2 \alpha}(x) \in D_{k}^{\circ}$ or $\Phi_{-2 \alpha}(x) \in D_{k}^{\circ}$ since $\epsilon<\alpha$ and for every $y \in X$ the set $\Phi_{[0,4 \alpha]}(y)$ can not intersect both the open bottom and the open top of $D_{k}$. Hence we can find $(M+2)$ pairwise distinct elements $k_{1}, k_{2}, \ldots, k_{M+2} \subseteq\{1,2, \ldots, 2 M+3\}$ such that $\Phi_{2 \alpha}(x) \in D_{k_{j}}^{\circ}$ holds for $j=1,2, \ldots, M+2$ or that $\Phi_{-2 \alpha}(x) \in D_{k_{j}}^{\circ}$ holds for $j=1,2, \ldots, M+2$. In both cases we get a contradiction to $\operatorname{dim}\left(\left\{D^{\circ} \mid D \in \mathcal{D}\right\}\right) \leq M$. This shows $\operatorname{dim}\left(\mathcal{U}_{K}\right) \leq$ $2 M+1$. Note that this bound depends only on $k_{G}$ and $d_{X}$ and is independent of $\alpha$. Because

$$
\operatorname{dim}\left(\mathcal{U}_{K} \cup \mathcal{U}_{\gamma} \cup \mathcal{U}_{X^{\mathbb{R}}}\right) \leq \operatorname{dim}\left(\mathcal{U}_{K}\right)+\operatorname{dim}\left(\mathcal{U}_{\gamma} \cup \mathcal{U}_{X^{\mathbb{R}}}\right)+1
$$

this implies that the dimension of

$$
\mathcal{U}:=\mathcal{U}_{K} \cup \mathcal{U}_{\gamma} \cup \mathcal{U}_{X^{\mathbb{R}}}
$$

is bounded by a number that depends only on $k_{G}, d_{X}$ and the $G$-action on an arbitrary small neighborhood of $X^{\mathbb{R}}$. Thus $\mathcal{U}$ is the required $\mathcal{V} \mathcal{C}$ yc-cover of $X$. This finishes the proof of Theorem 1.4.

Remark 5.3 In Convention 1.3 we assumed that the number of closed orbits, which are not stationary and whose period is $\leq C$, of the flow induced on $G \backslash X$ is finite for every $C>0$. This assumption can be replace with the following less restrictive assumption.

There is a number $N$ such that for every $\gamma>0$ there is a $G$-invariant collection $\mathcal{U}$ of open $\mathcal{V} \mathcal{C}$ c-subsets of $X$ such that for each $x \in X_{\leq \gamma}$ there is $U \in \mathcal{U}$ such that $\Phi_{[-\gamma, \gamma]} \subset U, \operatorname{dim}(\mathcal{U}) \leq N$ and $G \backslash \mathcal{U}$ is finite.

The proof of Theorem 1.4 given above clearly also works under this less restrictive assumption. This might be useful in nonpositively curved situations. 


\section{Mineyev's flow space}

\subsection{Hyperbolic complexes, double difference and Gromov product}

We collect some basic concepts such as hyperbolic complexes, double difference and Gromov product which are all taken from the paper by Mineyev [14] and which we will need for our purposes.

A simplicial complex is called uniformly locally finite if there exists a number $N$ such that any element in the 0 -skeleton $X_{0}$ occurs as vertex of at most $N$ simplices.

Let $X$ be a simplicial complex. Given any metric $d$ on its 0 -skeleton, one can extend it to a metric $\tilde{d}$ on $X$ as follows. Given points $u_{k}$ for $k=1,2$ in $X$, we can find vertices $x_{k}[0], x_{k}[1], x_{k}\left[n_{k}\right]$ such that $u_{k}$ belongs to the simplex with vertices $x_{k}[0]$, $x_{k}[1], \ldots, x_{k}\left[n_{k}\right]$. There are unique numbers $\alpha_{k}[0], \alpha_{k}[1], \ldots, \alpha_{k}\left[n_{k}\right]$ in $[0,1]$ with $\sum_{i_{k}=0}^{n_{k}} \alpha_{k}\left[i_{k}\right]=1$ such that $u_{k}$ is given by $\sum_{i_{k}=0}^{n_{k}} \alpha_{k}\left[i_{k}\right] \cdot x_{k}\left[n_{k}\right]$. Then define

$$
\tilde{d}\left(u_{1}, u_{2}\right)=\sum_{i_{1}=0}^{n_{1}} \sum_{i_{2}=0}^{n_{2}} \alpha_{1}\left[i_{1}\right] \cdot \alpha_{2}\left[i_{2}\right] \cdot d\left(x_{1}\left[i_{1}\right], x_{2}\left[i_{2}\right]\right) .
$$

This is a well-defined metric extending $d$ such that each simplex with the metric induced by $\tilde{d}$ is homeomorphic to the standard simplex.

Given a connected simplicial complex, define a metric $d_{0}$ on its 0 -skeleton by defining $d_{0}(x, y)$ as the minimum of the numbers $n \geq 0$ such that there is a sequence of vertices $x=x_{0}, x_{1}, \ldots, x_{n}=y$ with the property that $x_{i}$ and $x_{i+1}$ are joint by an edge for $i=0,1, \ldots, n-1$. The word metric $d_{\text {word }}$ on a connected simplicial complex $X$ is the metric $\tilde{d}_{0}$.

A metric complex $(X, d)$ is a connected uniformly locally finite simplicial complex $X$ equipped with its word metric $d=d_{\text {word }}$. A hyperbolic complex $X$ is a metric complex $(X, d)$ such that $(X, d)$ is $\delta$-hyperbolic in the sense of Gromov for some $\delta>0$ (see Gromov [10] and Bridson, Haefliger and Buch [5, Definition III.H.1.1]). Let $\partial X$ be the boundary and $\bar{X}=X \cup \partial X$ be the compactification of the hyperbolic complex $X$ in the sense of Gromov [10; 5, III.H.3].

Mineyev [14, 6.1] constructs for a hyperbolic metric complex $(X, d)$ a new metric $\widehat{D}$ with certain properties (see [14, Lemma 2.7 on page 449 and Theorem 32 on page 446]). For instance $\hat{D}$ is quasi-isometric to the word metric $d_{\text {word }}$. For a simplicial map $f: X \rightarrow X$ the following conditions are equivalent: (i) $f$ is a simplicial automorphism, (ii) $f$ is a simplicial automorphism preserving the word metric $d_{\text {word }}$, (iii) $f$ is a 
simplicial automorphism preserving the metric $\widehat{D}$. Define for $a, a^{\prime}, b, b^{\prime} \in X$ the double difference to be the real number

$$
\left\langle a, a^{\prime} \mid b, b^{\prime}\right\rangle:=\frac{1}{2} \cdot\left(\widehat{D}(a, b)+\widehat{D}\left(a^{\prime}, b^{\prime}\right)-\widehat{D}\left(a^{\prime}, b\right)-\widehat{D}\left(a, b^{\prime}\right)\right) .
$$

Recall that the Gromov product for $a, b, c \in X$ is defined to be the positive real number

$$
\langle a \mid b\rangle_{c}:=\frac{1}{2} \cdot(\widehat{D}(a, c)+\widehat{D}(b, c)-\widehat{D}(a, b)) .
$$

Define the subset

$$
S(\bar{X}) \subseteq\left\{\left(a, a^{\prime}, b, b^{\prime}\right) \in \bar{X} \times \bar{X} \times \bar{X} \times \bar{X}\right\}=\bar{X}^{4}
$$

to consist of those quadruples $\left(a, a^{\prime}, b, b^{\prime}\right)$ satisfying

$$
\begin{gathered}
a, b \in \partial X \Rightarrow a \neq b ; \\
a, b^{\prime} \in \partial X \Rightarrow a^{\prime} \neq b ; \\
a^{\prime}, b \in \partial X \Rightarrow a \neq b^{\prime} \\
a^{\prime}, b^{\prime} \in \partial X \Rightarrow a^{\prime} \neq b^{\prime} .
\end{gathered}
$$

Let

$$
T(\bar{X}):=\{(a, b, c)|\bar{X} \times \bar{X} \times \bar{X}| c \in \partial X \Rightarrow(a \neq c \text { and } b \neq c)\} .
$$

We equip $S(\bar{X}) \subseteq \bar{X}^{4}$ and $T(\bar{X}) \subseteq \bar{X}^{3}$ with the subspace topology. The following result is a special case of [14, Theorem 35 on page 448 and Theorem 36 on page 452]. (We only need and want to consider the case where the double difference takes values in $\mathbb{R}$.)

Theorem 6.3 (Mineyev) Let $(X, d)$ be a hyperbolic complex. Then the double difference of (6.1) extends to a continuous function invariant under simplicial automorphisms of $X$

$$
\langle-,-\mid-,-\rangle: S(\bar{X}) \rightarrow \mathbb{R}
$$

satisfying

(i) $\left\langle a, a^{\prime} \mid b, b^{\prime}\right\rangle=\left\langle b, b^{\prime} \mid a, a^{\prime}\right\rangle$;

(ii) $\left\langle a, a^{\prime} \mid b, b^{\prime}\right\rangle=-\left\langle a^{\prime}, a \mid b, b^{\prime}\right\rangle=-\left\langle a, a^{\prime} \mid b^{\prime}, b\right\rangle$;

(iii) $\left\langle a, a \mid b, b^{\prime}\right\rangle=\left\langle a, a^{\prime} \mid b, b\right\rangle=0$;

(iv) $\left\langle a, a^{\prime} \mid b, b^{\prime}\right\rangle+\left\langle a^{\prime}, a^{\prime \prime} \mid b, b^{\prime}\right\rangle=\left\langle a, a^{\prime \prime} \mid b, b^{\prime}\right\rangle$;

(v) $\langle a, b \mid c, x\rangle+\langle c, a \mid b, x\rangle+\langle b, c \mid a, x\rangle=0$; 
The Gromov product of (6.2) extends to a continuous function invariant under simplicial automorphisms of $X$

$$
\langle-\mid-\rangle_{-}: T(\bar{X}) \rightarrow[0, \infty]
$$

satisfying

(i) $\langle a \mid b\rangle_{c}=\infty \Leftrightarrow(c \in \partial X)$ or $(a, b \in \partial X$ and $a=b)$;

(ii) $\langle a, b \mid x, y\rangle=\langle b \mid x\rangle_{a}-\langle b \mid y\rangle_{a}$ for $a \in X$ and $(a, b, x, y) \in S(\bar{X})$.

We will often use the rules appearing in Theorem 6.3 tacitly.

Another important ingredient will be the following result due to Mineyev [14, Proposition 38 on page 453].

Proposition 6.4 (Mineyev) Let $(X, d)$ be a hyperbolic complex. Then there exist constants $\lambda \in\left(e^{-1}, 1\right)$ and $T \in[0, \infty)$ depending only on $X$ such that for all $a, b, c, u \in$ $\bar{X}$ satisfying

we have

$$
\begin{aligned}
& (a, c, u, b) \in S(\bar{X}) ; \\
& (b, c, u, a) \in S(\bar{X}) ;
\end{aligned}
$$

$$
\max \{\langle a, c \mid u, b\rangle,\langle b, c \mid u, a\rangle\} \geq T
$$

$$
\begin{aligned}
(u, c, a, b) & \in S(\bar{X}) \\
|\langle u, c \mid a, b\rangle| & \leq \lambda^{\max \{\langle a, c \mid u, b\rangle,\langle b, c \mid u, a\rangle\}} .
\end{aligned}
$$

\subsection{Two auxiliary functions}

In the sequel we will use the following two functions for $\alpha, \beta \in \overline{\mathbb{R}}:=\mathbb{R} \bigsqcup\{-\infty, \infty\}$ with $\alpha \leq \beta$.

$$
\begin{array}{r}
\theta_{[\alpha, \beta]}: \overline{\mathbb{R}} \rightarrow[\alpha, \beta] \\
\Theta_{[\alpha, \beta]}: \overline{\mathbb{R}} \rightarrow[\alpha, \beta]
\end{array}
$$

which are defined by

$$
\begin{aligned}
& \theta_{[\alpha, \beta]}(t):= \begin{cases}\alpha & \text { if }-\infty \leq t \leq \alpha ; \\
t & \text { if } \alpha \leq t \leq \beta ; \\
\beta & \text { if } \beta \leq t \leq \infty,\end{cases} \\
& \Theta_{[\alpha, \beta]}(t):= \begin{cases}-\infty & \text { if }-\infty=t=\alpha, \\
\alpha+e^{t-\alpha} / 2-e^{t-\beta} / 2 & \text { if }-\infty \leq t \leq \alpha,-\infty<\alpha<\infty ; \\
t+e^{\alpha-t} / 2-e^{t-\beta} / 2 & \text { if } \alpha \leq t \leq \beta,-\infty<t<\infty ; \\
\beta+e^{\alpha-t} / 2-e^{\beta-t} / 2 & \text { if } \beta \leq t \leq \infty,-\infty<\beta<\infty ; \\
\infty & \text { if } t=\beta=\infty .\end{cases}
\end{aligned}
$$


Here and in the sequel we use the convention that for $r, s \in \mathbb{R}$ the expressions $r+s$, $r \cdot s$ and $e^{r}$ are defined as usual and furthermore

$$
\begin{array}{ll}
r+\infty=\infty+r=\infty & \text { for } r \in \mathbb{R} \\
r-\infty=-\infty+r=-\infty & \text { for } r \in \mathbb{R} ; \\
e^{-\infty}=0 & \\
e^{\infty}=\infty & \\
| \pm \infty|=\infty &
\end{array}
$$

The function $\Theta_{[\alpha, \beta]}$ agrees with the function denoted by $\theta^{\prime}[\alpha, \beta ; \cdot]$ in Mineyev [14, Section 1.6]. We equip $\overline{\mathbb{R}}$ with the topology uniquely determined by the properties that $\mathbb{R} \subseteq \overline{\mathbb{R}}$ is an open subset, the subspace topology on $\mathbb{R} \subseteq \overline{\mathbb{R}}$ is the standard topology and a fundamental system for open neighborhoods of $\infty$ is $\{(R, \infty) \cup\{\infty\} \mid R \in \mathbb{R}\}$ and of $-\infty$ is $\{(-\infty, R) \cup\{-\infty\} \mid R \in \mathbb{R}\}$.

The elementary proof of the following basic properties of $\Theta_{[\alpha, \beta]}$ is left to the reader.

Lemma 6.7 Suppose that $\alpha<\beta$. Then:

(i) We have for $t \in \mathbb{R}$

$$
\Theta_{[\alpha, \beta]}(t)=\int_{-\infty}^{\infty} \frac{\theta_{[\alpha, \beta]}(t+s)}{2 \cdot e^{|s|}} d s=\theta_{[\alpha, \beta]}(t)+e^{-|\alpha-t|} / 2-e^{-|\beta-t|} / 2 ;
$$

(ii) The restriction of $\Theta_{[\alpha, \beta]}$ to $\mathbb{R}$ is a homeomorphism $\mathbb{R} \cong(\alpha, \beta)$ which is a $C^{1}$-function. Its first derivative is the continuous function

$$
t \in \mathbb{R} \mapsto \begin{cases}e^{t-\alpha} / 2-e^{t-\beta} / 2 & \text { if }-\infty<t \leq \alpha \\ 1-e^{\alpha-t} / 2-e^{t-\beta} / 2 & \text { if } \alpha \leq t \leq \beta \\ -e^{\alpha-t} / 2+e^{\beta-t} / 2 & \text { if } \beta \leq t<\infty\end{cases}
$$

(iii) The function $\Theta_{[\alpha, \beta]}$ is strictly monotone increasing. The function $\theta_{[\alpha, \beta]}$ is monotone increasing;

(iv) The function $\Theta_{[\alpha, \beta]}$ is nonexpanding, ie, $\left|\Theta_{[\alpha, \beta]}(t)-\Theta_{[\alpha, \beta]}(s)\right| \leq|t-s|$ for $t, s \in \overline{\mathbb{R}}$. The same is true for $\theta_{[\alpha, \beta]}$;

(v) The map $\Theta_{[\alpha, \beta]}: \overline{\mathbb{R}} \rightarrow[\alpha, \beta]$ is a homeomorphism;

(vi) We have for $t \in \overline{\mathbb{R}}$ and $s \in \mathbb{R}$

$$
\begin{aligned}
\theta_{[\alpha+s, \beta+s]}(t+s) & =\theta_{[\alpha, \beta]}(t)+s ; \\
\Theta_{[\alpha+s, \beta+s]}(t+s) & =\Theta_{[\alpha, \beta]}(t)+s ;
\end{aligned}
$$


(vii) Consider $\alpha_{0}, \alpha_{1}, \beta_{0}, \beta_{1} \in \overline{\mathbb{R}}$ such that $\alpha_{i} \leq \beta_{i}$ for $i=0,1,\left(\alpha_{0}=-\infty \Leftrightarrow \alpha_{1}=\right.$ $-\infty)$ and $\left(\beta_{0}=\infty \Leftrightarrow \beta_{1}=\infty\right)$ holds. Put

$$
C:= \begin{cases}\max \left\{\left|\alpha_{1}-\alpha_{0}\right|,\left|\beta_{1}-\beta_{0}\right|\right\} & \text { if } \alpha_{0}, \alpha_{1}, \beta_{0}, \beta_{1} \in \mathbb{R} \\ \left|\alpha_{1}-\alpha_{0}\right| & \text { if } \alpha_{0}, \alpha_{1}, \in \mathbb{R}, \beta_{0}=\beta_{1}=\infty ; \\ \left|\beta_{1}-\beta_{0}\right| & \text { if } \alpha_{0}=\alpha_{1}=-\infty, \beta_{0}, \beta_{1}, \in \mathbb{R} ; \\ 0 & \text { if } \alpha_{0}=\alpha_{1}=-\infty, \beta_{0}=\beta_{1}=\infty .\end{cases}
$$

Then we get for all $t \in \mathbb{R}$ that

$$
\begin{aligned}
\left|\theta_{\left[\alpha_{1}, \beta_{1}\right]}(t)-\theta_{\left[\alpha_{0}, \beta_{0}\right]}(t)\right| & \leq C ; \\
\left|\Theta_{\left[\alpha_{1}, \beta_{1}\right]}(t)-\Theta_{\left[\alpha_{0}, \beta_{0}\right]}(t)\right| & \leq C ;
\end{aligned}
$$

(viii) If $\alpha \leq t$, then

$$
\Theta_{[\alpha, \beta]}(t)-\Theta_{[-\infty, \beta]}(t)=e^{\alpha-t} / 2
$$

If $\beta \geq t$, then

$$
\Theta_{[\alpha, \infty]}(t)-\Theta_{[\alpha, \beta]}(t)=e^{t-\beta} / 2 ;
$$

(ix) Consider $\alpha, \beta \in \overline{\mathbb{R}}$ with $-\infty<\alpha<\beta$ and $t \in \mathbb{R}$.

If $\Theta_{[\alpha, \beta]}(t) \leq(\alpha+\beta) / 2$, then

$$
t \leq \min \left\{\beta, \Theta_{[\alpha, \beta]}(t)+1 / 2\right\} .
$$

If $\Theta_{[\alpha, \beta]}(t) \geq(\alpha+\beta) / 2$, then

$$
t \geq \max \left\{\alpha, \Theta_{[\alpha, \beta]}(t)-1 / 2\right\} .
$$

\subsection{The construction of the flow space}

Let $(X, d)$ be a hyperbolic complex. We want to define the associated flow space, ie a metric space $F S(X)$ together with a flow, following Mineyev [14]. (It is the same as the half open symmetric join $* \bar{X}$ constructed by Mineyev [14, Section 8.3].) The underlying set is

$$
\begin{aligned}
F S(X):=\{(a, b, t) \in \bar{X} \times \bar{X} \times \overline{\mathbb{R}} \mid(a \in \partial X \Rightarrow t \neq-\infty) \\
\\
\quad \text { and }(b \in \partial X \Rightarrow t \neq \infty) \text { and }(a, b \in \partial X \Rightarrow a \neq b)\} / \sim,
\end{aligned}
$$

where we identify $(a, b,-\infty) \sim\left(a, b^{\prime},-\infty\right),(a, b, \infty) \sim\left(a^{\prime}, b, \infty\right)$, and $(a, a, t) \sim$ $\left(a, a, t^{\prime}\right)$. In the sequel we will denote for $(a, b, t) \in \bar{X} \times \bar{X} \times \overline{\mathbb{R}}$ which satisfies $a \in \partial X \Rightarrow t \neq-\infty, b \in \partial X \Rightarrow t \neq \infty$ and $a, b \in \partial X \Rightarrow a \neq b$ its class in FSX again by $(a, b, t)$. 
From now on we fix a base point $x_{0} \in X$. The metric on $F S(X)$ will depend on this choice.

Define the map

$$
\begin{aligned}
l_{x_{0}}: X \times F S(X) & \rightarrow \mathbb{R} \\
(u,(a, b, t)) & \mapsto\langle a \mid b\rangle_{u}+\left|\theta_{\left[-\left\langle b \mid x_{0}\right\rangle_{a},\left\langle a \mid x_{0}\right\rangle_{b}\right]}(t)-\left\langle a, b \mid u, x_{0}\right\rangle\right| .
\end{aligned}
$$

It is easy to check that it is compatible with the equivalence relation appearing in the definition of $F S(X)$.

Definition 6.10 Define a pseudometric on $F S(X)$

$$
\begin{gathered}
d_{F S, x_{0}}^{\times}=d_{F S(X), x_{0}}^{\times}: F S(X) \times F S(X) \rightarrow \mathbb{R}, \\
(a, b, t),(c, d, s) \mapsto \sup _{u \in X}\left|l_{x_{0}}(u,(a, b, t))-l_{x_{0}}(u,(c, d, s))\right| .
\end{gathered}
$$

Recall that a pseudometric satisfies the same axioms as a metric except that the condition $d(x, y)=0 \Rightarrow x=y$ is dropped. The proof that this definition makes sense and yields a pseudometric $d_{F S, x_{0}}^{\times}$is given in Mineyev [14, Theorem 44 on page 459].

Lemma 6.11 The inclusion $X \rightarrow F S(X), x \mapsto(x, x, 0)$ is an isometric embedding with respect to the metric $\widehat{D}$ on $X$ and the pseudometric $d_{F S, x_{0}}^{\times}$on $F S(X)$.

Proof We compute for $u \in X$ and $x \in X$ and $t \in \overline{\mathbb{R}}$

$$
\begin{aligned}
l_{x_{0}}(u,(x, x, t)) & =\langle x \mid x\rangle_{u}+\left|\theta_{\left[-\left\langle x \mid x_{0}\right\rangle_{x},\left\langle x, x_{0}\right\rangle_{x}\right]}(t)-\left\langle x, x \mid u, x_{0}\right\rangle\right| \\
& =\widehat{D}(x, u)+\left|\theta_{[0,0]}(t)-0\right| \\
& =\widehat{D}(u, x) .
\end{aligned}
$$

Consider $a, b \in X$. Since by the triangle inequality $|\widehat{D}(u, a)-\widehat{D}(u, b)| \leq \widehat{D}(a, b)$ and $|\widehat{D}(b, a)-\widehat{D}(b, b)|=\widehat{D}(a, b)$ holds, we conclude

$$
\begin{aligned}
d_{F S, x_{0}}^{\times}((a, a, t),(b, b, s)) & =\sup _{u \in X}\left|l_{x_{0}}(u,(a, a, t))-l_{x_{0}}(u,(b, b, s))\right| \\
& =\sup _{u \in X}|\widehat{D}(u, a)-\widehat{D}(u, b)| \\
& =\widehat{D}(a, b) .
\end{aligned}
$$

The canonical $\mathbb{R}$-action on $\overline{\mathbb{R}}$

$$
\phi: \mathbb{R} \times \overline{\mathbb{R}}, \quad(\tau, t) \mapsto \phi_{\tau}(t)
$$


is defined by $\phi_{\tau}(t)=t+\tau$, if $t \in \mathbb{R}, \phi_{\tau}(-\infty)=-\infty$ and $\phi_{\tau}(\infty)=\infty$. This $\mathbb{R}$-action on $\overline{\mathbb{R}}$ together with the trivial $\mathbb{R}$-action on $\bar{X} \times \bar{X}$ yields an action of $\mathbb{R}$ on $\bar{X} \times \bar{X} \times \overline{\mathbb{R}}$ which in turn induces an $\mathbb{R}$-action

$$
\phi: \mathbb{R} \times F S(X) \rightarrow F S(X), \quad(\tau,(a, b, t)) \mapsto\left(a, b, \phi_{\tau}(t)\right) .
$$

For $a, b \in \bar{X}$ we define the line $(a, b)_{F S(X)}$ to be the set of points $\{(a, b, t) \mid t \in \mathbb{R}\}$. Obviously $(a, b)_{F S(X)}$ is a transitive free $\mathbb{R}$-set if $a \neq b$.

Next we construct the desired metric from the pseudometric above.

Definition 6.14 Define a metric on $F S(X)$

$$
\begin{aligned}
d_{F S(X), x_{0}}: F S(X) \times F S(X) & \rightarrow \mathbb{R}, \\
(a, b, t),(c, d, s) & \mapsto \int_{\mathbb{R}} \frac{d_{F S, x_{0}}^{\times}\left(\phi_{\tau}(a, b, t), \phi_{\tau}(c, d, s)\right)}{2 \cdot e^{|\tau|}} d \tau .
\end{aligned}
$$

Obviously $d_{F S, x_{0}}$ inherits from $d_{F S, x_{0}}^{\times}$the properties of a pseudometric. The proof that $d_{F S, x_{0}}$ is a metric can be found in [14, Theorem 14 on page 426 and Theorem 45 on page 459].

Lemma 6.15 The inclusion $X \rightarrow F S(X)$ is an isometric embedding with respect to the metric $\hat{D}$ on $X$ and the metric $d_{F S, x_{0}}$ on $F S(X)$.

Proof We compute for $u \in X$ and $x \in X$ and $t \in \overline{\mathbb{R}}$ using Lemma 6.11.

$$
\begin{aligned}
d_{F S, x_{0}}((a, a, t),(b, b, s)) & =\int_{\mathbb{R}} \frac{d_{F S, x_{0}}^{\times}\left(\phi_{\tau}(a, a, t), \phi_{\tau}(b, b, s)\right)}{2 \cdot e^{|\tau|}} d \tau \\
& =\int_{\mathbb{R}} \frac{d_{F S, x_{0}}^{\times}((a, a, t+\tau),(b, b, s+\tau))}{2 \cdot e^{|\tau|}} d \tau \\
& =\int_{\mathbb{R}} \frac{\widehat{D}(a, b)}{2 \cdot e^{|\tau|}} d \tau \\
& =\widehat{D}(a, b) \cdot \int_{\mathbb{R}} \frac{1}{2 \cdot e^{|\tau|}} d \tau \\
& =\widehat{D}(a, b) .
\end{aligned}
$$

In the sequel we will consider $X$ as a subspace of $F S(X)$ by the isometric embedding appearing in Lemma 6.15. 
Let $f: X \rightarrow X$ be an isometry, ie a bijection respecting the word metric $d$ on $X$. It extends uniquely to a homeomorphism $\bar{f}: \bar{X} \rightarrow \bar{X}$ and induces an isometry with respect to the metric $d_{F S(X), x_{0}}$

$F S(f): F S(X) \rightarrow F S(X), \quad(a, b, t) \mapsto\left(\bar{f}(a), \bar{f}(b), t+\left\langle a, b \mid x_{0}, f^{-1}\left(x_{0}\right)\right\rangle\right.$.

(Already $d_{F S, x_{0}}^{\times}$is invariant under this map by a straight-forward calculation that uses Lemma 6.7 (vi).) We have $F S(g \circ f)=F S(g) \circ F S(f)$ and $F S($ id $)=$ id. In particular a $G$-action on $X$ by isometries with respect to the word metric extends to a $G$-action on $F S(X)$ by isometries with respect to the metric $d_{F S(X), x_{0}}$.

Next we compute the pseudometric $d_{F S}^{\times}$and the metric $d_{F S}$ on a line.

Lemma 6.16 We get for $(a, b, t),(a, b, s) \in F S(X)$ and a given base point $x_{0} \in X$

$$
\begin{aligned}
& d_{F S, x_{0}}^{\times}((a, b, t),(a, b, s))=\left|\theta_{\left[-\left\langle b \mid x_{0}\right\rangle_{a},\left\langle a \mid x_{0}\right\rangle_{b}\right]}(t)-\theta_{\left[-\left\langle b \mid x_{0}\right\rangle_{a},\left\langle a \mid x_{0}\right\rangle_{b}\right]}(s)\right| ; \\
& d_{F S, x_{0}}((a, b, t),(a, b, s))=\left|\Theta_{\left[-\left\langle b \mid x_{0}\right\rangle_{a},\left\langle a \mid x_{0}\right\rangle_{b}\right]}(t)-\Theta_{\left[-\left\langle b \mid x_{0}\right\rangle_{a},\left\langle a \mid x_{0}\right\rangle_{b}\right]}(s)\right| .
\end{aligned}
$$

Proof We have

$$
\begin{aligned}
& d_{F S, x_{0}}^{\times}((a, b, t),(a, b, s)) \\
& =\sup _{u \in X}\left|l_{x_{0}}(u,(a, b, t))-l_{x_{0}}(u,(a, b, s))\right| \\
& =\sup _{u \in X} \mid\left(\langle a \mid b\rangle_{u}+\left|\theta_{\left[-\left\langle b \mid x_{0}\right\rangle_{a},\left\langle a \mid x_{0}\right\rangle_{b}\right]}(t)-\left\langle a, b \mid u, x_{0}\right\rangle\right|\right) \\
& \quad-\left(\langle a \mid b\rangle_{u}+\left|\theta_{\left[-\left\langle b \mid x_{0}\right\rangle_{a},\left\langle a \mid x_{0}\right\rangle_{b}\right]}(s)-\left\langle a, b \mid u, x_{0}\right\rangle\right|\right) \mid \\
& =\sup _{u \in X}|| \theta_{\left[-\left\langle b \mid x_{0}\right\rangle_{a},\left\langle a \mid x_{0}\right\rangle_{b}\right]}(t)-\left\langle a, b \mid u, x_{0}\right\rangle|-| \theta_{\left[-\left\langle b \mid x_{0}\right\rangle_{a},\left\langle a \mid x_{0}\right\rangle_{b}\right]}(s)-\left\langle a, b \mid u, x_{0}\right\rangle|| .
\end{aligned}
$$

We conclude from the triangle inequality

$$
\begin{aligned}
& || \theta_{\left[-\left\langle b \mid x_{0}\right\rangle_{a},\left\langle a \mid x_{0}\right\rangle_{b}\right]}(t)-\left\langle a, b \mid u, x_{0}\right\rangle|-| \theta_{\left[-\left\langle b \mid x_{0}\right\rangle_{a},\left\langle a \mid x_{0}\right\rangle_{b}\right]}(s)-\left\langle a, b \mid u, x_{0}\right\rangle|| \\
& \leq\left|\theta_{\left[-\left\langle b \mid x_{0}\right\rangle_{a},\left\langle a \mid x_{0}\right\rangle_{b}\right]}(t)-\left\langle a, b \mid u, x_{0}\right\rangle-\left(\theta_{\left[-\left\langle b \mid x_{0}\right\rangle_{a},\left\langle a \mid x_{0}\right\rangle_{b}\right]}(s)-\left\langle a, b \mid u, x_{0}\right\rangle\right)\right| \\
& \leq\left|\theta_{\left[-\left\langle b \mid x_{0}\right\rangle_{a},\left\langle a \mid x_{0}\right\rangle_{b}\right]}(t)-\theta_{\left[-\left\langle b \mid x_{0}\right\rangle_{a},\left\langle a \mid x_{0}\right\rangle_{b}\right]}(s)\right|,
\end{aligned}
$$

and we get for $u=a$

$$
\begin{aligned}
& \| \theta_{\left[-\left\langle b \mid x_{0}\right\rangle_{a},\left\langle a \mid x_{0}\right\rangle_{b}\right]}(t)-\left\langle a, b \mid a, x_{0}\right\rangle|-| \theta_{\left[-\left\langle b \mid x_{0}\right\rangle_{a},\left\langle a \mid x_{0}\right\rangle_{b}\right]}(s)-\left\langle a, b \mid a, x_{0}\right\rangle|| \\
& =|| \theta_{\left[-\left\langle b \mid x_{0}\right\rangle_{a},\left\langle a \mid x_{0}\right\rangle_{b}\right]}(t)-\left(-\left\langle b \mid x_{0}\right\rangle_{a}\right)|-| \theta_{\left[-\left\langle b \mid x_{0}\right\rangle_{a},\left\langle a \mid x_{0}\right\rangle_{b}\right]}(s)-\left(-\left\langle b \mid x_{0}\right\rangle_{a}\right) \| \\
& =\left|\theta_{\left[-\left\langle b \mid x_{0}\right\rangle_{a},\left\langle a \mid x_{0}\right\rangle_{b}\right]}(t)-\theta_{\left[-\left\langle b \mid x_{0}\right\rangle_{a},\left\langle a \mid x_{0}\right\rangle_{b}\right]}(s)\right| .
\end{aligned}
$$

This implies

$$
d_{F S, x_{0}}^{\times}((a, b, t),(a, b, s))=\left|\theta_{\left[-\left\langle b \mid x_{0}\right\rangle_{a},\left\langle a \mid x_{0}\right\rangle_{b}\right]}(t)-\theta_{\left[-\left\langle b \mid x_{0}\right\rangle_{a},\left\langle a \mid x_{0}\right\rangle_{b}\right]}(s)\right| .
$$


We prove the claim for $d_{F S, x_{0}}$ only in the case $t \geq s$, the case $t \leq s$ is analogous. Then $t+\tau \geq s+\tau$ holds for all $\tau \in \mathbb{R}$. Since both $\theta_{\left[-\left\langle b \mid x_{0}\right\rangle_{a},\left\langle a \mid x_{0}\right\rangle_{b}\right]}$ and $\Theta_{\left[-\left\langle b \mid x_{0}\right\rangle_{a},\left\langle a \mid x_{0}\right\rangle_{b}\right]}$ are monotone increasing, we conclude for all $\tau \in \mathbb{R}$,

$$
\begin{aligned}
& \theta_{\left[-\left\langle b \mid x_{0}\right\rangle_{a},\left\langle a \mid x_{0}\right\rangle_{b}\right]}(t+\tau)-\theta_{\left[-\left\langle b \mid x_{0}\right\rangle_{a},\left\langle a \mid x_{0}\right\rangle_{b}\right]}(s+\tau) \\
& =\left|\theta_{\left[-\left\langle b \mid x_{0}\right\rangle_{a},\left\langle a \mid x_{0}\right\rangle_{b}\right]}(t+\tau)-\theta_{\left[-\left\langle b \mid x_{0}\right\rangle_{a},\left\langle a \mid x_{0}\right\rangle_{b}\right]}(s+\tau)\right| ; \\
& \Theta_{\left[-\left\langle b \mid x_{0}\right\rangle_{a},\left\langle a \mid x_{0}\right\rangle_{b}\right]}(t+\tau)-\Theta_{\left[-\left\langle b \mid x_{0}\right\rangle_{a},\left\langle a \mid x_{0}\right\rangle_{b}\right]}(s+\tau) \\
& =\left|\Theta_{\left[-\left\langle b \mid x_{0}\right\rangle_{a},\left\langle a \mid x_{0}\right\rangle_{b}\right]}(t+\tau)-\Theta_{\left[-\left\langle b \mid x_{0}\right\rangle_{a},\left\langle a \mid x_{0}\right\rangle_{b}\right]}(s+\tau)\right| .
\end{aligned}
$$

Now we get

$$
\begin{aligned}
& d_{F S, x_{0}}((a, b, t),(a, b, s)) \\
& =\int_{\mathbb{R}} \frac{d_{F S, x_{0}}^{\times}\left(\phi_{\tau}((a, b, t)), \phi_{\tau}(a, b, s)\right)}{2 \cdot e^{|\tau|}} d \tau \\
& =\int_{\mathbb{R}} \frac{\left.d_{F S, x_{0}}^{\times}((a, b, t+\tau)),(a, b, s+\tau)\right)}{2 \cdot e^{|\tau|}} d \tau \\
& =\int_{\mathbb{R}} \frac{\left|\theta_{\left[-\left\langle b \mid x_{0}\right\rangle_{a},\left\langle a \mid x_{0}\right\rangle_{b}\right]}(t+\tau)-\theta_{\left[-\left\langle b \mid x_{0}\right\rangle_{a},\left\langle a \mid x_{0}\right\rangle_{b}\right]}(s+\tau)\right|}{2 \cdot e^{|\tau|}} d \tau \\
& =\int_{\mathbb{R}} \frac{\theta_{\left[-\left\langle b \mid x_{0}\right\rangle_{a},\left\langle a \mid x_{0}\right\rangle_{b}\right]}(t+\tau)-\theta_{\left[-\left\langle b \mid x_{0}\right\rangle_{a},\left\langle a \mid x_{0}\right\rangle_{b}\right]}(s+\tau)}{2 \cdot e^{|\tau|}} d \tau \\
& =\int_{\mathbb{R}} \frac{\theta_{\left[-\left\langle b \mid x_{0}\right\rangle_{a},\left\langle a \mid x_{0}\right\rangle_{b}\right]}(t+\tau)}{2 \cdot e^{|\tau|}} d \tau-\int_{\mathbb{R}} \frac{\theta_{\left[-\left\langle b \mid x_{0}\right\rangle_{a},\left\langle a \mid x_{0}\right\rangle_{b}\right]}(s+\tau)}{2 \cdot e^{|\tau|}} d \tau \\
& =\Theta_{\left[-\left\langle b \mid x_{0}\right\rangle_{a},\left\langle a \mid x_{0}\right\rangle_{b}\right]}(t)-\Theta_{\left[-\left\langle b \mid x_{0}\right\rangle_{a},\left\langle a \mid x_{0}\right\rangle_{b}\right]}(s) \\
& =\left|\Theta_{\left[-\left\langle b \mid x_{0}\right\rangle_{a},\left\langle a \mid x_{0}\right\rangle_{b}\right]}(t)-\Theta_{\left[-\left\langle b \mid x_{0}\right\rangle_{a},\left\langle a \mid x_{0}\right\rangle_{b}\right]}(s)\right| .
\end{aligned}
$$

This finishes the proof of Lemma 6.16.

Remark 6.17 We have fixed a base point $x_{0} \in X$. For a different base point $x_{1} \in X$ there is a canonical isometry $\left(F S(X), d_{F S, x_{0}}\right) \rightarrow\left(F S(X), d_{F S, x_{1}}\right)$ defined by $(a, b, t) \mapsto$ $\left(a, b, t+\left\langle a, b \mid x_{0}, x_{1}\right\rangle\right)$. (Of course this isometry appeared already when we defined $F S(f)$ for an isometry $f: X \rightarrow X$.) Using these isometries and a colimit over all choices of base points it is possible to give a canonical construction of the metric space $F S(X)$ without choosing a base point. However, then we do no longer have canonical coordinates in $F S(X)$, ie to make sense out of $(a, b, t) \in F S(X)$ we would still need to pick a base point. Since the base-point free formulation is not directly relevant for our applications, we do not give any details here.

Remark 6.18 As pointed out before, $F S(X)$ and $* \bar{X}$ agree as topological spaces. But it should be noted that the construction of the metric $d_{F S, x_{0}}$ on $F S(X)$ differs slightly 
from the metric $d_{*}$ constructed by Mineyev on $* \bar{X}$. The definitions of $l_{x_{0}}$ in (6.9) and of $l(u, x)$ in [14, Definition 10 on page 422] do not quite agree. There Mineyev uses a different parametrization to the effect that his formula translates to replacing $\theta$ by $\Theta$ in (6.9). (The point $\llbracket a, b ; s \rrbracket_{x_{0}} \in * \bar{X}$ corresponds to $\left(a, b, \Theta_{\left[-\left\langle b \mid x_{0}\right\rangle_{a},\left\langle a \mid x_{0}\right\rangle_{b}\right]}(s)\right)$ in our parametrization, because $\llbracket a, b ; s \rrbracket_{x_{0}}^{\prime}=\llbracket a, b ; \Theta_{\left[-\left\langle b \mid x_{0}\right\rangle_{a},\left\langle a \mid x_{0}\right\rangle_{b}\right]}(s) \rrbracket_{x_{0}}$ is used in [14, Section 2.3] to identify the models $X$ and $X$.) However, this difference is not important. All results of [14] that we will use are also valid with this minor variation. Moreover we remark that since $\left|\theta_{\left[-\left\langle b \mid x_{0}\right\rangle_{a},\left\langle a \mid x_{0}\right\rangle_{b}\right]}(t)-\Theta_{\left[-\left\langle b \mid x_{0}\right\rangle_{a},\left\langle a \mid x_{0}\right\rangle_{b}\right]}(t)\right| \leq 1$ for all $t \in \mathbb{R}$, it is easy to check that the identification of $F S(X)$ and $* \bar{X}$ is a quasi-isometry with respect to $d_{F S, x_{0}}$ and $d_{*}$.

\section{Flow estimates}

In this section we prove the main exponential flow estimate for $F S(X)$. Recall that we have fixed a base point $x_{0} \in X$.

Theorem 7.1 (Exponential flow estimate) Let $\lambda \in\left(e^{-1}, 1\right)$ and $T \in[0, \infty)$ be the constants depending only on $X$ which appear in Proposition 6.4. Consider $a, b, c \in \bar{X}$ such that $a, c \in \partial X \Rightarrow a \neq c$ and $b, c \in \partial X \Rightarrow b \neq c$ holds. Let $t, s, \tau \in \mathbb{R}$. Put

$$
\begin{gathered}
\tau_{0}=t-s-\left\langle a, b \mid c, x_{0}\right\rangle ; \\
N=2+\frac{2}{\lambda^{T} \cdot(-\ln (\lambda))} .
\end{gathered}
$$

Then we get

$$
d_{F S, x_{0}}\left(\phi_{\tau}(a, c, t), \phi_{\tau+\tau_{0}}(b, c, s)\right) \leq \frac{N}{1-\ln (\lambda)^{2}} \cdot \lambda^{\left(t-\left\langle a, c \mid b, x_{0}\right\rangle\right)} \cdot \lambda^{\tau} .
$$

For the sphere bundle of the universal cover of a strictly negatively curved manifold estimates as above are classical results and have been used in algebraic $K$-theory by Farrell and Jones [7]. Compare also the work of Bartels, Farrell, Jones and Reich [2, Proposition 14.2]. There only $c \in \partial X$ is considered and $\tau_{0}$ is chosen to ensure that $\phi_{\tau}(a, c, t)$ and $\phi_{\tau+\tau_{0}}(b, c, s)$ both lie on the same horosphere around $c$.

As mentioned in the introduction, the proof of Theorem 7.1 is strongly based on ideas due to Mineyev [14, Theorem 57 on page 468].

We also will use the following basic flow estimate.

Lemma 7.2 We get for $(a, b, t),(c, d, s) \in F S(X)$ and $\tau \in \mathbb{R}$,

$$
d_{F S, x_{0}}\left(\phi_{\tau}(a, b, t), \phi_{\tau}(c, d, s)\right) \leq e^{|\tau|} \cdot d_{F S, x_{0}}((a, b, t),(c, d, s)) .
$$


Proof We compute

$$
\begin{aligned}
& d_{F S, x_{0}}\left(\phi_{\tau}(a, b, t), \phi_{\tau}(c, d, s)\right) \\
& =\int_{\mathbb{R}} \frac{d_{F S, x_{0}}^{\times}\left(\phi_{\sigma}\left(\phi_{\tau}(a, b, t)\right), \phi_{\sigma}\left(\phi_{\tau}(c, d, s)\right)\right)}{2 \cdot e^{|\sigma|}} d \sigma \\
& =\int_{\mathbb{R}} \frac{d_{F S, x_{0}}^{\times}\left(\phi_{\sigma+\tau}(a, b, t), \phi_{\sigma+\tau}(c, d, s)\right)}{2 \cdot e^{|\sigma|}} d \sigma \\
& =\int_{\mathbb{R}} \frac{d_{F S, x_{0}}^{\times}\left(\phi_{\sigma}(a, b, t), \phi_{\sigma}(c, d, s)\right)}{2 \cdot e^{|\sigma-\tau|}} d \sigma \\
& \leq \int_{\mathbb{R}} \frac{d_{F S, x_{0}}^{\times}\left(\phi_{\sigma}(a, b, t), \phi_{\sigma}(c, d, s)\right)}{2 \cdot e^{|\sigma|-|\tau|}} d \sigma \\
& \leq e^{|\tau|} \cdot \int_{\mathbb{R}} \frac{d_{F S, x_{0}}^{\times}\left(\phi_{\sigma}(a, b, t), \phi_{\sigma}(c, d, s)\right)}{2 \cdot e^{|\sigma|}} d \sigma \\
& =e^{|\tau|} \cdot d_{F S, x_{0}}((a, b, t),(c, d, s)) .
\end{aligned}
$$

We record the following result due to Mineyev[14, Proposition 48 on page 460].

Theorem 7.3 The map

$$
(\bar{X} \times \bar{X}-\Delta(\bar{X})) \times \mathbb{R} \stackrel{\cong}{\rightarrow} F S(X)-X, \quad((a, b), t) \mapsto(a, b, t)
$$

is a homeomorphism, where $\Delta(\bar{X}) \subseteq \bar{X} \times \bar{X}$ is the diagonal.

\subsection{Flow estimates for the pseudo metric}

The goal of this subsection is to prove the version of Theorem 7.1 for the pseudometric $d_{F S, x_{0}}^{\times}$.

Theorem 7.4 Let $\lambda \in\left(e^{-1}, 1\right)$ and $T \in[0, \infty)$ be the constants depending only on $X$ which appear in Proposition 6.4. Consider $a, b, c \in \bar{X}$ such that $a, c \in \partial X \Rightarrow a \neq c$ and $b, c \in \partial X \Rightarrow b \neq c$ holds. Let $t, s, \tau \in \mathbb{R}$. Put

$$
\begin{aligned}
& \tau_{0}=t-s-\left\langle a, b \mid c, x_{0}\right\rangle \\
& N=2+\frac{2}{\lambda^{T} \cdot(-\ln (\lambda))}
\end{aligned}
$$

Then we get

$$
d_{F S, x_{0}}^{\times}\left(\phi_{\tau}(a, c, t), \phi_{\tau+\tau_{0}}(b, c, s)\right) \leq N \cdot \lambda^{t+\tau-\left\langle a, c \mid b, x_{0}\right\rangle} .
$$


Its proof needs some preparations and is then done in several steps.

We begin with the trivial case $a=b$.

Lemma 7.6 Consider the situation appearing in Theorem 7.4. If $a=b$ then (7.5) holds.

Proof Since

$$
\tau_{0}=t-s-\left\langle a, b \mid c, x_{0}\right\rangle=t-s-\left\langle a, a \mid c, x_{0}\right\rangle=t-s,
$$

we get for all $\tau \in \mathbb{R}$

$$
\phi_{\tau}(a, c, t)=(a, c, t+\tau)=\left(b, c, s+\tau+\tau_{0}\right)=\phi_{\tau+\tau_{0}}(b, c, s)
$$

and hence

$$
d_{F S, x_{0}}^{\times}\left(\phi_{\tau}(a, c, t), \phi_{\tau+\tau_{0}}(b, c, s)\right)=0 .
$$

So we can make in the sequel the additional assumption that $a \neq b$. This has the advantage that the expressions $\left\langle a, c \mid b, x_{0}\right\rangle,\left\langle b, c \mid a, x_{0}\right\rangle,\langle a, c \mid u, b\rangle$ and $\langle b, c \mid u, a\rangle$ for $u \in X$ which will appear below are well defined elements in $\mathbb{R}$.

Lemma 7.7 Define $\tau, \tau_{0}, a, b, c, x_{0}$ as in Theorem 7.4. Suppose that $a, b \in \partial X \Rightarrow$ $a \neq b$. Then:

(i) We have

$$
\begin{aligned}
\left\langle a \mid x_{0}\right\rangle_{c}-\left\langle a, c \mid b, x_{0}\right\rangle & =\left\langle b \mid x_{0}\right\rangle_{c}-\left\langle b, c \mid a, x_{0}\right\rangle=\langle a \mid b\rangle_{c} ; \\
t+\tau-\left\langle a, c \mid b, x_{0}\right\rangle & =s+\tau+\tau_{0}-\left\langle b, c \mid a, x_{0}\right\rangle ; \\
\left\langle a \mid x_{0}\right\rangle_{c}-(t+\tau) & =\left\langle b \mid x_{0}\right\rangle_{c}-\left(s+\tau+\tau_{0}\right) ; \\
-\left\langle c \mid x_{0}\right\rangle_{a}-\left\langle a, c \mid b, x_{0}\right\rangle & =-\langle c \mid b\rangle_{a} ; \\
-\left\langle c \mid x_{0}\right\rangle_{b}-\left\langle b, c \mid a, x_{0}\right\rangle & =-\langle c \mid a\rangle_{b} ; \\
\max \{\langle a, c \mid u, b\rangle,\langle b, c \mid u, a\rangle\} & \leq\left\langle a \mid x_{0}\right\rangle_{c}-\left\langle a, c \mid b, x_{0}\right\rangle \quad \text { for } u \in X ;
\end{aligned}
$$

(ii) If

$$
\begin{gathered}
t+\tau \geq-\left\langle c \mid x_{0}\right\rangle_{a} ; \\
s+\tau+\tau_{0} \geq-\left\langle c \mid x_{0}\right\rangle_{b}, \\
\theta_{\left[-\left\langle c \mid x_{0}\right\rangle_{a},\left\langle a \mid x_{0}\right\rangle_{c}\right]}(t+\tau)-\left\langle a, c \mid b, x_{0}\right\rangle \\
=\theta_{\left[-\left\langle c \mid x_{0}\right\rangle_{b},\left\langle b \mid x_{0}\right\rangle_{c}\right]}\left(s+\tau_{0}+\tau\right)-\left\langle b, c \mid a, x_{0}\right\rangle ;
\end{gathered}
$$

then 
(iii) If we assume

$$
\begin{gathered}
t+\tau \geq-\left\langle c \mid x_{0}\right\rangle_{a} ; \\
\theta_{\left[-\left\langle c \mid x_{0}\right\rangle_{a},\left\langle a \mid x_{0}\right\rangle_{c}\right]}(t+\tau)-\left\langle a, c \mid b, x_{0}\right\rangle<\max \{\langle a, c \mid u, b\rangle,\langle b, c \mid u, a\rangle\}, \\
\text { then } \quad \theta_{\left[-\left\langle c \mid x_{0}\right\rangle_{a},\left\langle a \mid x_{0}\right\rangle_{c}\right]}(t+\tau)=t+\tau .
\end{gathered}
$$

Proof (i) Notice for the sequel that $\left\langle a \mid a^{\prime}\right\rangle_{c}=\infty$ if $c \in \partial X$. Hence the first equality, the third equality and the last inequality are true for trivial reasons if $c \in \partial X$.

We compute for $c \in X$

$$
\begin{aligned}
\left\langle a \mid x_{0}\right\rangle_{c}-\left\langle a, c \mid b, x_{0}\right\rangle & =\left\langle a \mid x_{0}\right\rangle_{c}+\left\langle c, a \mid b, x_{0}\right\rangle \\
& =\left\langle a \mid x_{0}\right\rangle_{c}+\left(\langle a \mid b\rangle_{c}-\left\langle a \mid x_{0}\right\rangle_{c}\right) \\
& =\langle a \mid b\rangle_{c},
\end{aligned}
$$

and analogously

$$
\left\langle b \mid x_{0}\right\rangle_{c}-\left\langle b, c \mid a, x_{0}\right\rangle=\langle b \mid a\rangle_{c}=\langle a \mid b\rangle_{c} .
$$

This proves the first equation.

The second follows from

$$
\begin{aligned}
s+\tau+\tau_{0}- & \left\langle b, c \mid a, x_{0}\right\rangle \\
& =s+\tau+t-s-\left\langle a, b \mid c, x_{0}\right\rangle-\left\langle b, c \mid a, x_{0}\right\rangle \\
& =\tau+t-\left\langle a, b \mid c, x_{0}\right\rangle-\left\langle b, c \mid a, x_{0}\right\rangle-\left\langle c, a \mid b, x_{0}\right\rangle+\left\langle c, a \mid b, x_{0}\right\rangle \\
& =\tau+t-\left\langle a, c \mid b, x_{0}\right\rangle .
\end{aligned}
$$

The third equation is a direct consequence of the first two if $c \in X$, and hence true for all $c \in \bar{X}$.

The proof of the fourth and fifth equation is analogous to the one of the first one.

Since for $c \in X$

$$
\begin{aligned}
& \langle a, c \mid u, b\rangle=\langle c, a \mid b, u\rangle=\langle a \mid b\rangle_{c}-\langle a \mid u\rangle_{c} \leq\langle a \mid b\rangle_{c} \\
& \text { and } \quad\langle b, c \mid u, a\rangle=\langle c, b \mid a, u\rangle=\langle b \mid a\rangle_{c}-\langle b \mid u\rangle_{c} \leq\langle b \mid a\rangle_{c}=\langle a \mid b\rangle_{c}
\end{aligned}
$$

holds, the last inequality follows from the first equality.

(ii) We begin with the case $t+\tau \geq\left\langle a \mid x_{0}\right\rangle_{c}$. Then we get from the third equation of assertion (i) that also $s+\tau+\tau_{0} \geq\left\langle b \mid x_{0}\right\rangle_{c}$ holds. We conclude from the definitions 
and the first equation of assertion (i)

$$
\begin{aligned}
\theta_{\left[-\left\langle c \mid x_{0}\right\rangle_{a},\left\langle a \mid x_{0}\right\rangle_{c}\right]}(t+\tau)-\left\langle a, c \mid b, x_{0}\right\rangle & =\left\langle a \mid x_{0}\right\rangle_{c}-\left\langle a, c \mid b, x_{0}\right\rangle \\
& =\left\langle b \mid x_{0}\right\rangle_{c}-\left\langle b, c \mid a, x_{0}\right\rangle \\
& =\theta_{\left[-\left\langle c \mid x_{0}\right\rangle_{b},\left\langle b \mid x_{0}\right\rangle_{c}\right]}\left(s+\tau_{0}+\tau\right)-\left\langle b, c \mid a, x_{0}\right\rangle .
\end{aligned}
$$

Next we treat the case $t+\tau \leq\left\langle a \mid x_{0}\right\rangle_{c}$. Then we get from the third equation of assertion (i) that also $s+\tau+\tau_{0} \leq\left\langle b \mid x_{0}\right\rangle_{c}$ holds. Since by assumption $t+\tau \geq-\left\langle c \mid x_{0}\right\rangle_{a}$ and $s+\tau+\tau_{0} \geq-\left\langle c \mid x_{0}\right\rangle_{b}$ holds, we conclude from the definitions and the second equation of assertion (i)

$$
\begin{aligned}
\theta_{\left[-\left\langle c \mid x_{0}\right\rangle_{a},\left\langle a \mid x_{0}\right\rangle_{c}\right]}(t+\tau)-\left\langle a, c \mid b, x_{0}\right\rangle & =t+\tau-\left\langle a, c \mid b, x_{0}\right\rangle \\
& =s+\tau+\tau_{0}-\left\langle b, c \mid a, x_{0}\right\rangle \\
& =\theta_{\left[-\left\langle c \mid x_{0}\right\rangle_{b},\left\langle b \mid x_{0}\right\rangle_{c}\right]}\left(s+\tau_{0}+\tau\right)-\left\langle b, c \mid a, x_{0}\right\rangle .
\end{aligned}
$$

(iii) Since the inequality in assertion (i) implies

$$
\begin{aligned}
\theta_{\left[-\left\langle c \mid x_{0}\right\rangle_{a},\left\langle a \mid x_{0}\right\rangle_{c}\right]}(t+\tau) & <\max \{\langle a, c \mid u, b\rangle,\langle b, c \mid u, a\rangle\}+\left\langle a, c \mid b, x_{0}\right\rangle \\
& \leq\left\langle a \mid x_{0}\right\rangle_{c},
\end{aligned}
$$

we get $t+\tau<\left\langle a \mid x_{0}\right\rangle_{c}$. Since we assume $-\left\langle c \mid x_{0}\right\rangle_{a} \leq t+\tau$, we conclude

$$
\theta_{\left[-\left\langle c \mid x_{0}\right\rangle_{a},\left\langle a \mid x_{0}\right\rangle_{c}\right]}(t+\tau)=t+\tau \text {. }
$$

This finishes the proof of Lemma 7.7.

The elementary proof of the next lemma is left to the reader.

Lemma 7.8 Consider numbers $\lambda \in\left(e^{-1}, 1\right)$ and $T \in[0, \infty)$. Put

$$
N:=2+\frac{2}{\lambda^{T} \cdot(-\ln (\lambda))}
$$

Then we get

$$
\begin{array}{lll}
2 & \leq N \\
2 \cdot\left(T-v+\lambda^{v}\right) & \leq N \cdot \lambda^{v} & \text { for all } v \leq T \\
v+N \cdot \lambda^{v-w} & \leq N \cdot \lambda^{-w} & \text { for } 0 \leq v \leq w .
\end{array}
$$

Lemma 7.9 Consider the situation appearing in Theorem 7.4. Suppose that $a, b \in$ $\partial X \Rightarrow a \neq b$. Suppose that

$$
\begin{aligned}
t+\tau & \geq-\left\langle c \mid x_{0}\right\rangle_{a} ; \\
s+\tau+\tau_{0} & \geq-\left\langle c \mid x_{0}\right\rangle_{b} .
\end{aligned}
$$


Then we get for all $u \in X$

$$
\begin{aligned}
& \left|l_{x_{0}}\left(u, \phi_{\tau}(a, c, t)\right)-l_{x_{0}}\left(u, \phi_{\tau+\tau_{0}}(b, c, s)\right)\right| \\
& \quad \begin{array}{r}
|\langle u, c \mid a, b\rangle+| \theta_{\left[-\left\langle c \mid x_{0}\right\rangle_{a},\left\langle a \mid x_{0}\right\rangle_{c}\right]}(t+\tau)-\left\langle a, c \mid b, x_{0}\right\rangle-\langle a, c \mid u, b\rangle \mid \\
-\left|\theta_{\left[-\left\langle c \mid x_{0}\right\rangle_{a},\left\langle a, x_{0}\right\rangle_{c}\right]}(t+\tau)-\left\langle a, c \mid b, x_{0}\right\rangle-\langle b, c \mid u, a\rangle\right| \mid .
\end{array}
\end{aligned}
$$

\section{Proof We compute}

$$
\begin{aligned}
& \left|l_{x_{0}}\left(u, \phi_{\tau}(a, c, t)\right)-l_{x_{0}}\left(u, \phi_{\tau+\tau_{0}}(b, c, s)\right)\right| \\
& =\left|l_{x_{0}}(u,(a, c, t+\tau))-l_{x_{0}}\left(u,\left(b, c, s+\tau+\tau_{0}\right)\right)\right| \\
& =\left|\langle a \mid c\rangle_{u}+\right| \theta_{\left[-\left\langle c \mid x_{0}\right\rangle_{a},\left\langle a \mid x_{0}\right\rangle_{c}\right]}(t+\tau)-\left\langle a, c \mid u, x_{0}\right\rangle \mid-\langle b \mid c\rangle_{u} \\
& \quad \quad-\left|\theta_{\left[-\left\langle c \mid x_{0}\right\rangle_{b},\left\langle b \mid x_{0}\right\rangle_{c}\right]}\left(s+\tau_{0}+\tau\right)-\left\langle b, c \mid u, x_{0}\right\rangle\right| \mid \\
& =\mid\langle c \mid a\rangle_{u}-\langle c \mid b\rangle_{u} \\
& \quad+\left|\theta_{\left[-\left\langle c \mid x_{0}\right\rangle_{a},\left\langle a \mid x_{0}\right\rangle_{c}\right]}(t+\tau)-\left\langle a, c \mid b, x_{0}\right\rangle+\left\langle a, c \mid b, x_{0}\right\rangle-\left\langle a, c \mid u, x_{0}\right\rangle\right| \\
& \quad-\left|\theta_{\left[-\left\langle c \mid x_{0}\right\rangle_{b},\left\langle b \mid x_{0}\right\rangle_{c}\right]}\left(s+\tau_{0}+\tau\right)-\left\langle b, c \mid a, x_{0}\right\rangle+\left\langle b, c \mid a, x_{0}\right\rangle-\left\langle b, c \mid u, x_{0}\right\rangle\right| \mid \\
& =|\langle u, c \mid a, b\rangle+| \theta_{\left[-\left\langle c \mid x_{0}\right\rangle_{a},\left\langle a \mid x_{0}\right\rangle_{c}\right]}(t+\tau)-\left\langle a, c \mid b, x_{0}\right\rangle-\langle a, c \mid u, b\rangle \mid \\
& \quad-\left|\theta_{\left[-\left\langle c \mid x_{0}\right\rangle_{b},\left\langle b \mid x_{0}\right\rangle_{c}\right]}\left(s+\tau_{0}+\tau\right)-\left\langle b, c \mid a, x_{0}\right\rangle-\langle b, c \mid u, a\rangle\right| \mid .
\end{aligned}
$$

Since we get from Lemma 7.7 (ii)

$\theta_{\left[-\left\langle c \mid x_{0}\right\rangle_{a},\left\langle a \mid x_{0}\right\rangle_{c}\right]}(t+\tau)-\left\langle a, c \mid b, x_{0}\right\rangle=\theta_{\left[-\left\langle c \mid x_{0}\right\rangle_{b},\left\langle b \mid x_{0}\right\rangle_{c}\right]}\left(s+\tau_{0}+\tau\right)-\left\langle b, c \mid a, x_{0}\right\rangle$.

Lemma 7.9 follows.

Lemma 7.10 Consider the situation appearing in Theorem 7.4. Suppose that $a, b \in$ $\partial X \Rightarrow a \neq b$. Suppose that

$$
\begin{aligned}
t+\tau & \geq-\left\langle c \mid x_{0}\right\rangle_{a} ; \\
s+\tau+\tau_{0} & \geq-\left\langle c \mid x_{0}\right\rangle_{b},
\end{aligned}
$$

and $\theta_{\left[-\left\langle c \mid x_{0}\right\rangle_{a},\left\langle a \mid x_{0}\right\rangle_{c}\right]}(t+\tau)-\left\langle a, c \mid b, x_{0}\right\rangle \geq \max \{\langle a, c \mid u, b\rangle,\langle b, c \mid u, a\rangle\}$.

Then we get for all $u \in X$

$$
\left|l_{x_{0}}\left(u, \phi_{\tau}(a, c, t)\right)-l_{x_{0}}\left(u, \phi_{\tau+\tau_{0}}(b, c, s)\right)\right|=0 .
$$


Proof We compute

$$
\begin{aligned}
& \left|\theta_{\left[-\left\langle c \mid x_{0}\right\rangle_{a},\left\langle a \mid x_{0}\right\rangle_{c}\right]}(t+\tau)-\left\langle a, c \mid b, x_{0}\right\rangle-\langle a, c \mid u, b\rangle\right| \\
& -\left|\theta_{\left[-\left\langle c \mid x_{0}\right\rangle_{a},\left\langle a \mid x_{0}\right\rangle_{c}\right]}(t+\tau)-\left\langle a, c \mid b, x_{0}\right\rangle-\langle b, c \mid u, a\rangle\right| \\
& =\left(\theta_{\left[-\left\langle c \mid x_{0}\right\rangle_{a},\left\langle a \mid x_{0}\right\rangle_{c}\right]}(t+\tau)-\left\langle a, c \mid b, x_{0}\right\rangle-\langle a, c \mid u, b\rangle\right) \\
& -\left(\theta_{\left[-\left\langle c \mid x_{0}\right\rangle_{a},\left\langle a \mid x_{0}\right\rangle_{c}\right]}(t+\tau)-\left\langle a, c \mid b, x_{0}\right\rangle-\langle b, c \mid u, a\rangle\right) \\
& =-\langle a, c \mid u, b\rangle+\langle b, c \mid u, a\rangle \text {. }
\end{aligned}
$$

This implies together with Lemma 7.9

$$
\begin{aligned}
\mid l_{x_{0}}\left(u, \phi_{\tau}(a, c, t)\right)-l_{x_{0}}\left(u, \phi_{\tau+\tau_{0}}\right. & (b, c, s)) \mid \\
& =|\langle u, c \mid a, b\rangle-\langle a, c \mid u, b\rangle+\langle b, c \mid u, a\rangle| \\
& =|\langle b, a \mid c, u\rangle+\langle a, c \mid b, u\rangle+\langle c, b \mid a, u\rangle| \\
& =0 .
\end{aligned}
$$

Lemma 7.11 Consider the situation appearing in Theorem 7.4. Suppose that $a, b \in$ $\partial X \Rightarrow a \neq b$. Suppose that

$$
\begin{aligned}
t+\tau & \geq-\left\langle c \mid x_{0}\right\rangle_{a} ; \\
s+\tau+\tau_{0} & \geq-\left\langle c \mid x_{0}\right\rangle_{b},
\end{aligned}
$$

and that

$$
\begin{aligned}
& \theta_{\left[-\left\langle c \mid x_{0}\right\rangle_{a},\left\langle a \mid x_{0}\right\rangle_{c}\right]}(t+\tau)-\left\langle a, c \mid b, x_{0}\right\rangle<\max \{\langle a, c \mid u, b\rangle,\langle b, c \mid u, a\rangle\} ; \\
& \theta_{\left[-\left\langle c \mid x_{0}\right\rangle_{a},\left\langle a \mid x_{0}\right\rangle_{c}\right]}(t+\tau)-\left\langle a, c \mid b, x_{0}\right\rangle \geq T .
\end{aligned}
$$

Then we get for all $u \in X$

$$
\left|l_{x_{0}}\left(u, \phi_{\tau}(a, c, t)\right)-l_{x_{0}}\left(u, \phi_{\tau+\tau_{0}}(b, c, s)\right)\right| \leq 2 \cdot \lambda^{t+\tau-\left\langle a, c \mid b, x_{0}\right\rangle} .
$$

Proof We get from Lemma 7.9

$$
\begin{aligned}
\mid l_{x_{0}} & \left(u, \phi_{\tau}(a, c, t)\right)-l_{x_{0}}\left(u, \phi_{\tau+\tau_{0}}(b, c, s)\right) \mid \\
= & |\langle u, c \mid a, b\rangle+| \theta_{\left[-\left\langle c \mid x_{0}\right\rangle_{a},\left\langle a \mid x_{0}\right\rangle_{c}\right]}(t+\tau)-\left\langle a, c \mid b, x_{0}\right\rangle-\langle a, c \mid u, b\rangle \mid \\
& \quad-\left|\theta_{\left[-\left\langle c \mid x_{0}\right\rangle_{a},\left\langle a \mid x_{0}\right\rangle_{c}\right]}(t+\tau)-\left\langle a, c \mid b, x_{0}\right\rangle-\langle b, c \mid u, a\rangle\right| \mid \\
\leq & |\langle u, c \mid a, b\rangle|+\mid\left(\theta_{\left[-\left\langle c \mid x_{0}\right\rangle_{a},\left\langle a \mid x_{0}\right\rangle_{c}\right]}(t+\tau)-\left\langle a, c \mid b, x_{0}\right\rangle-\langle a, c \mid u, b\rangle\right) \\
& \quad-\left(\theta_{\left[-\left\langle c \mid x_{0}\right\rangle_{a},\left\langle a \mid x_{0}\right\rangle_{c}\right]}(t+\tau)-\left\langle a, c \mid b, x_{0}\right\rangle-\langle b, c \mid u, a\rangle\right) \mid \\
= & |\langle u, c \mid a, b\rangle|+|-\langle a, c \mid u, b\rangle+\langle b, c \mid u, a\rangle| \\
= & |\langle u, c \mid a, b\rangle|+|\langle c, a \mid u, b\rangle+\langle a, u \mid c, b\rangle|
\end{aligned}
$$




$$
\begin{aligned}
& =|\langle u, c \mid a, b\rangle|+|\langle u, c \mid a, b\rangle| \\
& =2 \cdot|\langle u, c \mid a, b\rangle| .
\end{aligned}
$$

Our assumptions imply $\max \{\langle a, c \mid u, b\rangle,\langle b, c| u, a\} \geq T$. We conclude from Proposition 6.4

$$
|\langle u, c \mid a, b\rangle| \leq \lambda^{\max \{\langle a, c \mid u, b\rangle,\langle b, c \mid u, a\rangle\}} .
$$

Thus we get using Lemma 7.7 (iii)

$$
\begin{aligned}
\left|l_{x_{0}}\left(u, \phi_{\tau}(a, c, t)\right)-l_{x_{0}}\left(u, \phi_{\tau+\tau_{0}}(b, c, s)\right)\right| & \leq 2 \cdot \lambda^{\max \{\langle a, c \mid u, b\rangle,\langle b, c \mid u, a\rangle\}} \\
& \leq 2 \cdot \lambda^{\theta_{\left[-\left\langle c \mid x_{0}\right\rangle a,\left\langle a \mid x_{0}\right\rangle c\right]}(t+\tau)-\left\langle a, c \mid b, x_{0}\right\rangle} \\
& =2 \cdot \lambda^{t+\tau-\left\langle a, c \mid b, x_{0}\right\rangle} .
\end{aligned}
$$

Lemma 7.12 Consider the situation appearing in Theorem 7.4. Suppose that $a, b \in$ $\partial X \Rightarrow a \neq b$. Suppose that

$$
\begin{aligned}
t+\tau & \geq-\left\langle c \mid x_{0}\right\rangle_{a} ; \\
s+\tau+\tau_{0} & \geq-\left\langle c \mid x_{0}\right\rangle_{b},
\end{aligned}
$$

and that

$$
\begin{aligned}
& \theta_{\left[-\left\langle c \mid x_{0}\right\rangle_{a},\left\langle a \mid x_{0}\right\rangle_{c}\right]}(t+\tau)-\left\langle a, c \mid b, x_{0}\right\rangle<\max \{\langle a, c \mid u, b\rangle,\langle b, c \mid u, a\rangle\} ; \\
& \theta_{\left[-\left\langle c \mid x_{0}\right\rangle_{a},\left\langle a \mid x_{0}\right\rangle_{c}\right]}(t+\tau)-\left\langle a, c \mid b, x_{0}\right\rangle<T .
\end{aligned}
$$

Then we get for all $u \in X$

$$
\left|l_{x_{0}}\left(u, \phi_{\tau}(a, c, t)\right)-l_{x_{0}}\left(u, \phi_{\tau+\tau_{0}}(b, c, s)\right)\right| \leq N \cdot \lambda^{t+\tau-\left\langle a, c \mid b, x_{0}\right\rangle} .
$$

Proof Since $\theta_{\left[-\left\langle c \mid x_{0}\right\rangle_{a},\left\langle a \mid x_{0}\right\rangle_{c}\right]}$ is monotone increasing and by Lemma 7.7 (i) and by assumption

$$
\begin{aligned}
\theta_{\left[-\left\langle c \mid x_{0}\right\rangle_{a},\left\langle a \mid x_{0}\right\rangle_{c}\right]}(t+\tau)-\left\langle a, c \mid b, x_{0}\right\rangle & <\min \{\max \{\langle a, c \mid u, b\rangle,\langle b, c \mid u, a\rangle\}, T\} ; \\
\max \{\langle a, c \mid u, b\rangle,\langle b, c \mid u, a\rangle\} & \leq\left\langle a \mid x_{0}\right\rangle_{c}-\left\langle a, c \mid b, x_{0}\right\rangle,
\end{aligned}
$$

holds, we can choose $\tau^{\prime} \in \mathbb{R}$ satisfying

$$
\begin{aligned}
\theta_{\left[-\left\langle c \mid x_{0}\right\rangle_{a},\left\langle a \mid x_{0}\right\rangle_{c}\right]}\left(t+\tau^{\prime}\right)-\left\langle a, c \mid b, x_{0}\right\rangle & =\min \{\max \{\langle a, c \mid u, b\rangle,\langle b, c \mid u, a\rangle\}, T\} ; \\
\tau & \leq \tau^{\prime} .
\end{aligned}
$$

In particular we have

$$
\begin{aligned}
t+\tau^{\prime} & \geq-\left\langle c \mid x_{0}\right\rangle_{a} ; \\
s+\tau^{\prime}+\tau_{0} & \geq-\left\langle c \mid x_{0}\right\rangle_{b} .
\end{aligned}
$$


Hence Lemma 7.10 and Lemma 7.11 imply

$$
\begin{aligned}
\left|l_{x_{0}}\left(u, \phi_{\tau^{\prime}}(a, c, t)\right)-l_{x_{0}}\left(u, \phi_{\tau^{\prime}+\tau_{0}}(b, c, s)\right)\right| & \leq 2 \cdot \lambda^{t+\tau^{\prime}-\left\langle a, c \mid b, x_{0}\right\rangle} \\
& \leq 2 \cdot \lambda^{t+\tau-\left\langle a, c \mid b, x_{0}\right\rangle} .
\end{aligned}
$$

We compute

$$
\begin{aligned}
& \left|l_{x_{0}}\left(u, \phi_{\tau}(a, c, t)\right)-l_{x_{0}}\left(u, \phi_{\tau+\tau_{0}}(b, c, s)\right)\right| \\
& =\mid l_{x_{0}}\left(u, \phi_{\tau}(a, c, t)\right)-l_{x_{0}}\left(u, \phi_{\tau^{\prime}}(a, c, t)\right) \\
& \quad+l_{x_{0}}\left(u, \phi_{\tau^{\prime}}(a, c, t)\right)-l_{x_{0}}\left(u, \phi_{\tau^{\prime}+\tau_{0}}(b, c, s)\right) \\
& \quad+l_{x_{0}}\left(u, \phi_{\tau^{\prime}+\tau_{0}}(b, c, s)\right)-l_{x_{0}}\left(u, \phi_{\tau+\tau_{0}}(b, c, s)\right) \mid \\
& \leq\left|l_{x_{0}}\left(u, \phi_{\tau}(a, c, t)\right)-l_{x_{0}}\left(u, \phi_{\tau^{\prime}}(a, c, t)\right)\right| \\
& \quad+\left|l_{x_{0}}\left(u, \phi_{\tau^{\prime}}(a, c, t)\right)-l_{x_{0}}\left(u, \phi_{\tau^{\prime}+\tau_{0}}(b, c, s)\right)\right| \\
& +\left|l_{x_{0}}\left(u, \phi_{\tau^{\prime}+\tau_{0}}(b, c, s)\right)-l_{x_{0}}\left(u, \phi_{\tau+\tau_{0}}(b, c, s)\right)\right| .
\end{aligned}
$$

We estimate using Lemma 7.7 (iii)

$$
\begin{aligned}
& \text { (7.15) }\left|l_{x_{0}}\left(u, \phi_{\tau}(a, c, t)\right)-l_{x_{0}}\left(u, \phi_{\tau^{\prime}}(a, c, t)\right)\right| \\
& =\mid\left(\langle a \mid c\rangle_{u}+\left|\theta_{\left[-\left\langle c \mid x_{0}\right\rangle_{a},\left\langle a \mid x_{0}\right\rangle_{c}\right]}(t+\tau)-\left\langle a, c \mid u, x_{0}\right\rangle\right|\right) \\
& -\left(\langle a \mid c\rangle_{u}+\left|\theta_{\left[-\left\langle c \mid x_{0}\right\rangle_{a},\left\langle a \mid x_{0}\right\rangle_{c}\right]}\left(t+\tau^{\prime}\right)-\left\langle a, c \mid u, x_{0}\right\rangle\right|\right) \mid \\
& =|| \theta_{\left[-\left\langle c \mid x_{0}\right\rangle_{a},\left\langle a \mid x_{0}\right\rangle_{c}\right]}(t+\tau)-\left\langle a, c \mid u, x_{0}\right\rangle \mid \\
& -\left|\theta_{\left[-\left\langle c \mid x_{0}\right\rangle_{a},\left\langle a \mid x_{0}\right\rangle_{c}\right]}\left(t+\tau^{\prime}\right)-\left\langle a, c \mid u, x_{0}\right\rangle\right| \mid \\
& \leq\left|\theta_{\left[-\left\langle c \mid x_{0}\right\rangle_{a},\left\langle a \mid x_{0}\right\rangle_{c}\right]}(t+\tau)-\theta_{\left[-\left\langle c \mid x_{0}\right\rangle_{a},\left\langle a \mid x_{0}\right\rangle_{c}\right]}\left(t+\tau^{\prime}\right)\right| \\
& =\mid\left(\theta_{\left[-\left\langle c \mid x_{0}\right\rangle_{a},\left\langle a \mid x_{0}\right\rangle_{c}\right]}(t+\tau)-\left\langle a, c \mid b, x_{0}\right\rangle\right) \\
& -\left(\theta_{\left[-\left\langle c \mid x_{0}\right\rangle_{a},\left\langle a \mid x_{0}\right\rangle_{c}\right]}\left(t+\tau^{\prime}\right)-\left\langle a, c \mid b, x_{0}\right\rangle\right) \mid \\
& =\mid\left(\theta_{\left[-\left\langle c \mid x_{0}\right\rangle_{a},\left\langle a \mid x_{0}\right\rangle_{c}\right]}(t+\tau)-\left\langle a, c \mid b, x_{0}\right\rangle\right) \\
& -\min \{\max \{\langle a, c \mid u, b\rangle,\langle b, c \mid u, a\rangle\}, T\} \mid \\
& =\min \{\max \{\langle a, c \mid u, b\rangle,\langle b, c \mid u, a\rangle\}, T\} \\
& -\left(\theta_{\left[-\left\langle c \mid x_{0}\right\rangle_{a},\left\langle a \mid x_{0}\right\rangle_{c}\right]}(t+\tau)-\left\langle a, c \mid b, x_{0}\right\rangle\right) \\
& =\min \{\max \{\langle a, c \mid u, b\rangle,\langle b, c \mid u, a\rangle\}, T\}-\left(t+\tau-\left\langle a, c \mid b, x_{0}\right\rangle\right) \\
& \leq T-\left(t+\tau-\left\langle a, c \mid b, x_{0}\right\rangle\right) \text {. }
\end{aligned}
$$


Analogously we get using Lemma 7.7 (ii)

$$
\begin{aligned}
& \left|l_{x_{0}}\left(u, \phi_{\tau+\tau_{0}}(b, c, s)\right)-l_{x_{0}}\left(u, \phi_{\tau^{\prime}+\tau_{0}}(b, c, s)\right)\right| \\
& =\mid\left(\langle b \mid c\rangle_{u}+\left|\theta_{\left[-\left\langle c \mid x_{0}\right\rangle_{b},\left\langle b \mid x_{0}\right\rangle_{c}\right]}\left(s+\tau+\tau_{0}\right)-\left\langle b, c \mid u, x_{0}\right\rangle\right|\right) \\
& -\left(\langle b \mid c\rangle_{u}+\left|\theta_{\left[-\left\langle c \mid x_{0}\right\rangle_{b},\left\langle b \mid x_{0}\right\rangle_{c}\right]}\left(s+\tau^{\prime}+\tau_{0}\right)-\left\langle b, c \mid u, x_{0}\right\rangle\right|\right) \mid \\
& =|| \theta_{\left[-\left\langle c \mid x_{0}\right\rangle_{b},\left\langle b \mid x_{0}\right\rangle_{c}\right]}\left(s+\tau+\tau_{0}\right)-\left\langle b, c \mid u, x_{0}\right\rangle \mid \\
& -\left|\theta_{\left[-\left\langle c \mid x_{0}\right\rangle_{b},\left\langle b \mid x_{0}\right\rangle_{c}\right]}\left(s+\tau^{\prime}+\tau_{0}\right)-\left\langle b, c \mid u, x_{0}\right\rangle\right| \mid \\
& \leq\left|\theta_{\left[-\left\langle c \mid x_{0}\right\rangle_{b},\left\langle b \mid x_{0}\right\rangle_{c}\right]}\left(s+\tau+\tau_{0}\right)-\theta_{\left[-\left\langle c \mid x_{0}\right\rangle_{b},\left\langle b \mid x_{0}\right\rangle_{c}\right]}\left(s+\tau^{\prime}+\tau_{0}\right)\right| \\
& =\mid\left(\theta_{\left[-\left\langle c \mid x_{0}\right\rangle_{b},\left\langle b \mid x_{0}\right\rangle_{c}\right]}\left(s+\tau+\tau_{0}\right)-\left\langle b, c \mid a, x_{0}\right\rangle\right) \\
& -\left(\theta_{\left[-\left\langle c \mid x_{0}\right\rangle_{b},\left\langle b, x_{0}\right\rangle_{c}\right]}\left(s+\tau^{\prime}+\tau_{0}\right)-\left\langle b, c \mid a, x_{0}\right\rangle\right) \mid \\
& =\mid\left(\theta_{\left[-\left\langle c \mid x_{0}\right\rangle_{a},\left\langle a \mid x_{0}\right\rangle_{c}\right]}(t+\tau)-\left\langle a, c \mid b, x_{0}\right\rangle\right) \\
& -\left(\theta_{\left[-\left\langle c \mid x_{0}\right\rangle_{a},\left\langle a \mid x_{0}\right\rangle_{c}\right]}\left(t+\tau^{\prime}\right)-\left\langle a, c \mid b, x_{0}\right\rangle\right) \mid \\
& \leq T-\left(t+\tau-\left\langle a, c \mid b, x_{0}\right\rangle\right) \text {. }
\end{aligned}
$$

Lemma 7.7 (iii) implies

$$
t+\tau-\left\langle a, c \mid b, x_{0}\right\rangle=\theta_{\left[-\left\langle c \mid x_{0}\right\rangle_{a},\left\langle a \mid x_{0}\right\rangle_{c}\right]}(t+\tau)-\left\langle a, c \mid b, x_{0}\right\rangle \leq T .
$$

Hence we conclude from Lemma 7.8 for $v=t+\tau-\left\langle a, c \mid b, x_{0}\right\rangle$

$$
2 \cdot\left(T-\left(t+\tau-\left\langle a, c \mid b, x_{0}\right\rangle\right)+\lambda^{t+\tau-\left\langle a, c \mid b, x_{0}\right\rangle}\right) \leq N \cdot \lambda^{t+\tau-\left\langle a, c \mid b, x_{0}\right\rangle} .
$$

Combining (7.13), (7.14), (7.15), (7.16) and (7.17) yields

$$
\begin{aligned}
\mid l_{x_{0}} & \left(u, \phi_{\tau}(a, c, t)\right)-l_{x_{0}}\left(u, \phi_{\tau+\tau_{0}}(b, c, s)\right) \mid \\
& \leq 2 \cdot\left(T-\left(t+\tau-\left\langle a, c \mid b, x_{0}\right\rangle\right)+\lambda^{t+\tau-\left\langle a, c \mid b, x_{0}\right\rangle}\right) \\
& \leq N \cdot \lambda^{t+\tau-\left\langle a, c \mid b, x_{0}\right\rangle}
\end{aligned}
$$

Lemma 7.18 Consider the situation appearing in Theorem 7.4. Suppose that $a, b \in$ $\partial X \Rightarrow a \neq b$. Suppose that

$$
\begin{aligned}
t+\tau & \geq-\left\langle c \mid x_{0}\right\rangle_{a} ; \\
s+\tau+\tau_{0} & \geq-\left\langle c \mid x_{0}\right\rangle_{b} .
\end{aligned}
$$

Then (7.5) holds. 
Proof This follows from Lemma 7.10, Lemma 7.11 (note that $2 \leq N$ by Lemma 7.8) and Lemma 7.12 since by definition

$$
\begin{aligned}
d_{F S, x_{0}}^{\times}\left(\phi_{\tau}(a, c, t),\right. & \left.\phi_{\tau+\tau_{0}}(b, c, s)\right) \\
& =\sup _{u \in X}\left|l_{x_{0}}\left(u, \phi_{\tau}(a, c, t)\right)-l_{x_{0}}\left(u, \phi_{\tau+\tau_{0}}(b, c, s)\right)\right| .
\end{aligned}
$$

Lemma 7.19 Consider the situation appearing in Theorem 7.4. Suppose that $a, b \in$ $\partial X \Rightarrow a \neq b$. Suppose that at least one of the following inequalities is true:

$$
\begin{aligned}
t+\tau & \leq-\left\langle c \mid x_{0}\right\rangle_{a} ; \\
s+\tau+\tau_{0} & \leq-\left\langle c \mid x_{0}\right\rangle_{b} .
\end{aligned}
$$

Then (7.5) holds.

Proof Put $\quad \tau^{\prime \prime}:=\max \left\{-\left\langle c \mid x_{0}\right\rangle_{a}-t,-\left\langle c \mid x_{0}\right\rangle_{b}-s-\tau_{0}\right\}$.

Since by assumption $\tau \leq-\left\langle c \mid x_{0}\right\rangle_{a}-t$ or $\tau \leq-\left\langle c \mid x_{0}\right\rangle_{b}-s-\tau_{0}$ holds, we must have

$$
\tau \leq \tau^{\prime \prime}
$$

We estimate

$$
\begin{aligned}
& d_{F S, x_{0}}^{\times}\left(\phi_{\tau}(a, c, t), \phi_{\tau+\tau_{0}}(b, c, s)\right) \\
& =d_{F S, x_{0}}^{\times}\left((a, c, t+\tau),\left(b, c, s+\tau+\tau_{0}\right)\right) \\
& \leq d_{F S, x_{0}}^{\times}\left((a, c, t+\tau),\left(a, c, t+\tau^{\prime \prime}\right)\right) \\
& \quad+d_{F S, x_{0}}^{\times}\left(\left(a, c, t+\tau^{\prime \prime}\right),\left(b, c, s+\tau^{\prime \prime}+\tau_{0}\right)\right) \\
& \quad+d_{F S, x_{0}}^{\times}\left(\left(b, c, s+\tau^{\prime \prime}+\tau_{0}\right),\left(b, c, s+\tau+\tau_{0}\right)\right) .
\end{aligned}
$$

Since

$$
\begin{array}{r}
t+\tau^{\prime \prime} \geq-\left\langle c \mid x_{0}\right\rangle_{a} ; \\
s+\tau^{\prime \prime}+\tau_{0} \geq-\left\langle c \mid x_{0}\right\rangle_{b},
\end{array}
$$

holds by definition of $\tau^{\prime \prime}$, we get from Lemma 7.18

$$
d_{F S, x_{0}}^{\times}\left(\left(a, c, t+\tau^{\prime \prime}\right),\left(b, c, s+\tau^{\prime \prime}+\tau_{0}\right)\right) \leq N \cdot \lambda^{t+\tau^{\prime \prime}-\left\langle a, c \mid b, x_{0}\right\rangle} .
$$

Next we want to show

$$
\begin{aligned}
& d_{F S, x_{0}}^{\times}\left((a, c, t+\tau),\left(a, c, t+\tau^{\prime \prime}\right)\right) \\
& \quad+d_{F S, x_{0}}^{\times}\left(\left(b, c, s+\tau^{\prime \prime}+\tau_{0}\right),\left(b, c, s+\tau+\tau_{0}\right)\right) \leq \tau^{\prime \prime}-\tau .
\end{aligned}
$$


Inspecting the definition of $\tau^{\prime \prime}$ we see that we have to consider two cases, namely,

and

$$
\begin{aligned}
& t+\tau^{\prime \prime}=-\left\langle c \mid x_{0}\right\rangle_{a} \text { and } s+\tau_{0}+\tau^{\prime \prime} \geq-\left\langle c \mid x_{0}\right\rangle_{b}, \\
& t+\tau^{\prime \prime} \geq-\left\langle c \mid x_{0}\right\rangle_{a} \text { and } s+\tau_{0}+\tau^{\prime \prime}=-\left\langle c \mid x_{0}\right\rangle_{b} .
\end{aligned}
$$

We only treat the first one, the second is completely analogous. From $t+\tau \leq t+$ $\tau^{\prime \prime}=-\left\langle c \mid x_{0}\right\rangle_{a}$ we conclude $\theta_{\left[-\left\langle c \mid x_{0}\right\rangle_{a},\left\langle a \mid x_{0}\right\rangle_{c}\right]}(t+\tau)=\theta_{\left[-\left\langle c \mid x_{0}\right\rangle_{b},\left\langle b \mid x_{0}\right\rangle_{c}\right]}\left(t+\tau^{\prime \prime}\right)$. Lemma 6.16 implies

$$
d_{F S, x_{0}}^{\times}\left((a, c, t+\tau),\left(a, c, t+\tau^{\prime \prime}\right)\right)=0 .
$$

We conclude from Lemma 6.16

$$
\begin{aligned}
d_{F S, x_{0}}^{\times} & \left(\left(b, c, s+\tau^{\prime \prime}+\tau_{0}\right),\left(b, c, s+\tau+\tau_{0}\right)\right) \\
& =\left|\theta_{\left[-\left\langle c \mid x_{0}\right\rangle_{b},\left\langle b \mid x_{0}\right\rangle_{c}\right]}\left(s+\tau^{\prime \prime}+\tau_{0}\right)-\theta_{\left[-\left\langle c \mid x_{0}\right\rangle_{b},\left\langle b \mid x_{0}\right\rangle_{c}\right]}\left(s+\tau+\tau_{0}\right)\right| \\
& \leq\left|\left(s+\tau^{\prime \prime}+\tau_{0}\right)-\left(s+\tau+\tau_{0}\right)\right| \\
& =\tau^{\prime \prime}-\tau .
\end{aligned}
$$

This finishes the proof of (7.22).

If we combine (7.20), (7.21) and (7.22), we get

$$
d_{F S, x_{0}}^{\times}\left(\phi_{\tau}(a, c, t), \phi_{\tau+\tau_{0}}(b, c, s)\right) \leq \tau^{\prime \prime}-\tau+N \cdot \lambda^{t+\tau^{\prime \prime}-\left\langle a, c \mid b, x_{0}\right\rangle} .
$$

We estimate

$$
-\left\langle c \mid x_{0}\right\rangle_{a}-t \leq\langle c \mid b\rangle_{a}-\left\langle c \mid x_{0}\right\rangle_{a}-t=\left\langle a, c \mid b, x_{0}\right\rangle-t,
$$

and for $b \in X$

$$
\begin{aligned}
& -\left\langle c \mid x_{0}\right\rangle_{b}-s-\tau_{0} \\
& =-\left\langle c \mid x_{0}\right\rangle_{b}-t+\left\langle a, b \mid c, x_{0}\right\rangle \\
& =\left\langle a, c \mid b, x_{0}\right\rangle-t-\left\langle a, c \mid b, x_{0}\right\rangle+\left\langle a, b \mid c, x_{0}\right\rangle-\left\langle c \mid x_{0}\right\rangle_{b} \\
& =\left\langle a, c \mid b, x_{0}\right\rangle-t+\left\langle c, a \mid b, x_{0}\right\rangle+\left\langle a, b \mid c, x_{0}\right\rangle+\left\langle b, c \mid a, x_{0}\right\rangle-\left\langle b, c \mid a, x_{0}\right\rangle-\left\langle c \mid x_{0}\right\rangle_{b} \\
& =\left\langle a, c \mid b, x_{0}\right\rangle-t-\left\langle b, c \mid a, x_{0}\right\rangle-\left\langle c \mid x_{0}\right\rangle_{b} \\
& =\left\langle a, c \mid b, x_{0}\right\rangle-t-\langle c \mid a\rangle_{b} \\
& \leq\left\langle a, c \mid b, x_{0}\right\rangle-t .
\end{aligned}
$$

This inequality holds for $b \in \partial X$ for trivial reasons. The last two inequalities imply

$$
\tau^{\prime \prime} \leq\left\langle a, c \mid b, x_{0}\right\rangle-t
$$

and hence

$$
0 \leq \tau^{\prime \prime}-\tau \leq-\left(t+\tau-\left\langle a, c \mid b, x_{0}\right\rangle\right)
$$


Lemma 7.8 applied to $v=\tau^{\prime \prime}-\tau$ and $w=-\left(t+\tau-\left\langle a, c \mid b, x_{0}\right\rangle\right)$ yields

$$
\tau^{\prime \prime}-\tau+N \cdot \lambda^{t+\tau^{\prime \prime}-\left\langle a, c \mid b, x_{0}\right\rangle} \leq N \cdot \lambda^{t+\tau-\left\langle a, c \mid b, x_{0}\right\rangle} .
$$

If we combine (7.23), (7.24), we get the desired inequality

$$
d_{F S, x_{0}}^{\times}\left(\phi_{\tau}(a, c, t), \phi_{\tau+\tau_{0}}(b, c, s)\right) \leq N \cdot \lambda^{t+\tau-\left\langle a, c \mid b, x_{0}\right\rangle} .
$$

Now Theorem 7.4 follows from Lemma 7.6, Lemma 7.18 and Lemma 7.19.

\subsection{Flow estimates for the metric}

Next we prove Theorem 7.1.

Proof We estimate using $e \cdot \lambda>1,0<e^{-1} \cdot \lambda<1$ and Theorem 7.4

$$
\begin{aligned}
d_{F S, x_{0}}\left(\phi_{\tau}(a, c, t), \phi_{\tau+\tau_{0}}(b, c, s)\right) \\
=\int_{-\infty}^{\infty} \frac{d_{F S, x_{0}}^{\times}\left(\phi_{\sigma}\left(\phi_{\tau}(a, c, t)\right), \phi_{\sigma}\left(\phi_{\tau+\tau_{0}}(b, c, s)\right)\right)}{2 \cdot e^{|\sigma|}} d \sigma \\
=\int_{-\infty}^{\infty} \frac{d_{F S}^{\times}\left(\phi_{\sigma+\tau}((a, c, t)), \phi_{\sigma+\tau+\tau_{0}}((b, c, s))\right)}{2 \cdot e^{|\sigma|}} d \sigma \\
\leq \int_{-\infty}^{\infty} \frac{N \cdot \lambda^{t+\sigma+\tau-\left\langle a, c \mid b, x_{0}\right\rangle}}{2 \cdot e^{|\sigma|}} d \sigma \\
=\int_{-\infty}^{0} \frac{N \cdot \lambda^{t+\sigma+\tau-\left\langle a, c \mid b, x_{0}\right\rangle}}{2 \cdot e^{|\sigma|}} d \sigma+\int_{0}^{\infty} \frac{N \cdot \lambda^{t+\sigma+\tau-\left\langle a, c \mid b, x_{0}\right\rangle}}{2 \cdot e^{|\sigma|}} d \sigma . \\
=\int_{-\infty}^{0} \frac{N \cdot \lambda^{t+\sigma+\tau-\left\langle a, c \mid b, x_{0}\right\rangle}}{2 \cdot e^{-\sigma}} d \sigma+\int_{0}^{\infty} \frac{N \cdot \lambda^{t+\sigma+\tau-\left\langle a, c \mid b, x_{0}\right\rangle}}{2 \cdot e^{\sigma}} d \sigma . \\
=\frac{N}{2} \cdot \lambda^{t+\tau-\left\langle a, c \mid b, x_{0}\right\rangle} \cdot\left(\int_{-\infty}^{0}(e \cdot \lambda)^{\sigma} d \sigma+\int_{0}^{\infty}\left(e^{-1} \cdot \lambda\right)^{\sigma} d \sigma\right) \\
\left.=\frac{N}{2} \cdot \lambda^{t+\tau-\left\langle a, c \mid b, x_{0}\right\rangle} \cdot\left(\left[\frac{(e \cdot \lambda)^{\sigma}}{\ln (e \cdot \lambda)}\right]_{-\infty}^{0}+\frac{\left(e^{-1} \cdot \lambda\right)^{\sigma}}{\ln \left(e^{-1} \cdot \lambda\right)}\right]_{0}^{\infty}\right) \\
=\frac{N}{2} \cdot \lambda^{t+\tau-\left\langle a, c \mid b, x_{0}\right\rangle} \cdot\left(\frac{1}{\ln (e \cdot \lambda)}+\frac{1}{-\ln \left(e^{-1} \cdot \lambda\right)}\right) \\
=\frac{N}{2} \cdot \lambda^{t+\tau-\left\langle a, c \mid b, x_{0}\right\rangle} \cdot\left(\frac{1}{1+\ln (\lambda)}+\frac{1}{1-\ln (\lambda)}\right) \\
=\frac{N}{1-\ln (\lambda)^{2} \cdot \lambda^{\left(t-\left\langle a, c \mid b, x_{0}\right\rangle\right)} \cdot \lambda^{\tau} .} \\
=
\end{aligned}
$$




\section{The flow estimates for the map $\iota$}

Let $X$ be a hyperbolic complex and $x_{0} \in X$ be a base point. We define a map

$$
\iota_{x_{0}}: X \times \bar{X} \rightarrow F S(X)
$$

by $\iota_{x_{0}}(a, c):=$

$$
\begin{cases}\left(a, c, \Theta_{\left[-\left\langle c \mid x_{0}\right\rangle_{a},\left\langle a \mid x_{0}\right\rangle_{c}\right]}^{-1}\left(\min \{2, \hat{D}(a, c) / 2\}-\left\langle c \mid x_{0}\right\rangle_{a}\right)\right) & c \in X \text { and } a \neq c \\ \left(a, c, \Theta_{\left[-\left\langle c \mid x_{0}\right\rangle_{a}, \infty\right]}^{-1}\left(2-\left\langle c \mid x_{0}\right\rangle_{a}\right)\right) & c \in \partial X \\ c=(c, c, 0) & \text { if } a=c .\end{cases}
$$

We remind the reader that for $a, c \in X$ we have $a=(a, a, 0)=(a, a,-\infty)=(a, c,-\infty)$ in $F S(X)$.

Remark 8.2 Because of Lemma 6.16 the point $\iota_{x_{0}}(a, c)$ is $c$ if $a=c$, is the unique point on the line $(a, c)_{F S(X)}$ whose distance with respect to $d_{F S, x_{0}}$ from $a$ is $\min \{2, \hat{D}(a, c) / 2\}$ if $c \in X$ and $a \neq c$, and is the unique point on the line $(a, c)_{F S(X)}$ whose distance with respect to $d_{F S, x_{0}}$ from $a$ is 2 if $c \in \partial X$.

Lemma 8.3 Consider $a, b \in X$ and $c \in \bar{X}$ with $a \neq c$ and $t \in \mathbb{R}$. Suppose

$$
d_{F S, x_{0}}((a, c, t),(a, a, 0))= \begin{cases}\min \{2, \hat{D}(a, c) / 2\} & c \in X \\ 2 & c \in \partial X .\end{cases}
$$

Then

$$
-\widehat{D}(a, b) \leq t-\left\langle a, c \mid b, x_{0}\right\rangle \leq 5 / 2 \text {. }
$$

Proof Note that $(a, a, 0)=(a, c,-\infty) \in F S(X)$. We conclude from Lemma 6.16, Lemma 7.7 (i) and Lemma 6.7 (vi)

$$
\begin{aligned}
& \Theta_{\left[-\langle c \mid b\rangle_{a},\langle a \mid b\rangle_{c}\right]}\left(t-\left\langle a, c \mid b, x_{0}\right\rangle\right) \\
& =\Theta_{\left[-\left\langle c \mid x_{0}\right\rangle_{a}-\left\langle a, c \mid b, x_{0}\right\rangle,\left\langle a \mid x_{0}\right\rangle_{c}-\left\langle a, c \mid b, x_{0}\right\rangle\right]}\left(t-\left\langle a, c \mid b, x_{0}\right\rangle\right) \\
& =\Theta_{\left[-\left\langle c \mid x_{0}\right\rangle_{a},\left\langle a \mid x_{0}\right\rangle_{c}\right]}(t)-\left\langle a, c \mid b, x_{0}\right\rangle \\
& =\Theta_{\left[-\left\langle c \mid x_{0}\right\rangle_{a},\left\langle a \mid x_{0}\right\rangle_{c}\right]}(t)-\left(-\left\langle c \mid x_{0}\right\rangle_{a}\right)+\left(-\left\langle c \mid x_{0}\right\rangle_{a}\right)-\left\langle a, c \mid b, x_{0}\right\rangle \\
& =\Theta_{\left[-\left\langle c \mid x_{0}\right\rangle_{a},\left\langle a \mid x_{0}\right\rangle_{c}\right]}(t)-\left(-\left\langle c \mid x_{0}\right\rangle_{a}\right)-\langle c \mid b\rangle_{a} \\
& =d_{F S, x_{0}}((a, c, t),(a, a, 0))-\langle c \mid b\rangle_{a} .
\end{aligned}
$$

If $c \in X$, we get by assumption

$$
d_{F S, x_{0}}((a, c, t),(a, a, 0)) \leq \frac{\widehat{D}(a, c)}{2}=\frac{\langle a \mid b\rangle_{c}-\left(-\langle c \mid b\rangle_{a}\right)}{2},
$$


and hence

$$
d_{F S, x_{0}}((a, c, t),(a, a, 0))-\langle c \mid b\rangle_{a} \leq \frac{\langle a \mid b\rangle_{c}+\left(-\langle c \mid b\rangle_{a}\right)}{2} .
$$

This inequality is true for $c \in \partial X$ for trivial reasons. Hence we get from (8.4)

$$
\Theta_{\left[-\langle c \mid b\rangle_{a},\langle a \mid b\rangle_{c}\right]}\left(t-\left\langle a, c \mid b, x_{0}\right\rangle\right) \leq \frac{-\langle c \mid b\rangle_{a}+\langle a \mid b\rangle_{c}}{2} .
$$

Lemma 6.7 (ix) together with (8.4) implies

$$
\begin{aligned}
t-\left\langle a, c \mid b, x_{0}\right\rangle & \leq \Theta_{\left[-\langle c \mid b\rangle_{a},\langle a \mid b\rangle_{c}\right]}\left(t-\left\langle a, c \mid b, x_{0}\right\rangle\right)+1 / 2 \\
& =d_{F S, x_{0}}((a, c, t),(a, a, 0))-\langle c \mid b\rangle_{a}+1 / 2 \\
& \leq d_{F S, x_{0}}((a, c, t),(a, a, 0))+1 / 2 \\
& \leq 2+1 / 2 \\
& =5 / 2 .
\end{aligned}
$$

Thus we have proven the upper bound $t-\left\langle a, c \mid b, x_{0}\right\rangle \leq 5 / 2$. It remains to show the lower bound $-\hat{D}(a, b) \leq t-\left\langle a, c \mid b, x_{0}\right\rangle$.

We conclude from the assumptions that

$$
d_{F S, x_{0}}((a, c, t),(a, a, 0))=\frac{\widehat{D}(a, c)}{2}=\frac{\langle a \mid b\rangle_{c}-\left(-\langle c \mid b\rangle_{a}\right)}{2} \text { and } c \in X
$$

or

$$
d_{F S, x_{0}}((a, c, t),(a, a, 0))=2
$$

holds. We begin with the first case. Then

$$
d_{F S, x_{0}}((a, c, t),(a, a, 0))-\langle c \mid b\rangle_{a} \geq \frac{\langle a \mid b\rangle_{c}+\left(-\langle c \mid b\rangle_{a}\right)}{2} .
$$

Lemma 6.7 (ix) together with (8.4) implies

$$
t-\left\langle a, c \mid b, x_{0}\right\rangle \geq-\langle c \mid b\rangle_{a} \geq-\widehat{D}(a, b) .
$$

Finally we treat the second case. Then (8.4) implies

$$
\Theta_{\left[-\langle c \mid b\rangle_{a},\langle a \mid b\rangle_{c}\right]}\left(t-\left\langle a, c \mid b, x_{0}\right\rangle\right) \geq 2-\langle c \mid b\rangle_{a} .
$$

Since for $u \leq-\langle c \mid b\rangle_{a}$ we have

$$
\Theta_{\left[-\langle c \mid b\rangle_{a},\langle a \mid b\rangle_{c}\right]}(u)=-\langle c \mid b\rangle_{a}+e^{u-\left(-\langle c \mid b\rangle_{a}\right)} / 2-e^{u-\langle a \mid b\rangle_{c}} / 2<2-\langle c \mid b\rangle_{a},
$$

we conclude

$$
t-\left\langle a, c \mid b, x_{0}\right\rangle \geq-\langle c \mid b\rangle_{a} \geq-\widehat{D}(a, b) .
$$

This finishes the proof of Lemma 8.3. 
Lemma 8.5 The map $\iota_{x_{0}}: X \times \bar{X} \rightarrow F S(X)$ from (8.1) is continuous. It is $\operatorname{Isom}(X)-$ equivariant with respect to the diagonal $\operatorname{Isom}(X)$-action on the source. For $x \in X$ the map $\iota_{x_{0}}(x,-): \bar{X} \rightarrow F S(X), \quad y \mapsto \iota_{x_{0}}(x, y)$ is injective.

Proof We only prove continuity, the other claims are straight-forward to check using Remark 8.2. Recall that $X$ and $F S(X)$ are metric spaces. By [5, Exercise III.H.3.18(4)] the space $\bar{X}$ is metrizable. Hence it suffices to check continuity for sequences. Consider sequences $\left(a_{n}\right)_{n \geq 0}$ in $X$ and $\left(c_{n}\right)_{n \geq 0}$ in $\bar{X}$ and points $a \in X$ and $c \in \bar{X}$ such that $\lim _{n \rightarrow \infty} a_{n}=a$ in $X$ and $\lim _{n \rightarrow \infty} c_{n}=c$ in $\bar{X}$ hold. We have to show $\lim _{n \rightarrow \infty} \iota_{x_{0}}\left(a_{n}, c_{n}\right)=\iota_{x_{0}}(a, c)$ in $F S(X)$.

Suppose that $a=c$. Then we can assume $c_{n} \in X$ and $\widehat{D}\left(a_{n}, c_{n}\right) / 2<2$ for $n \geq 0$ and $\lim _{n \rightarrow \infty} \widehat{D}\left(a_{n}, c_{n}\right)=0$. This implies by the construction of $\iota_{x_{0}}$ that

$$
\lim _{n \rightarrow \infty} d_{F S, x_{0}}\left(a_{n}, \iota_{x_{0}}\left(a_{n}, c_{n}\right)\right)=\lim _{n \rightarrow \infty} d_{F S, x_{0}}\left(a_{n}, c_{n}\right) / 2=0
$$

and hence

$$
\lim _{n \rightarrow \infty} \iota_{x_{0}}\left(a_{n}, c_{n}\right)=\lim _{n \rightarrow \infty} a_{n}=a=c=\iota_{x_{0}}(a, c) .
$$

Hence we can assume without loss of generality that $a \neq c$ and $a_{n} \neq c_{n}$ for all $n \geq 0$ holds.

For $n \geq 0$ put $\alpha_{n}=-\left\langle c_{n} \mid x_{0}\right\rangle_{a_{n}}$ and $\beta_{n}=\left\langle a_{n} \mid x_{0}\right\rangle_{c_{n}}$. Put $\alpha=-\left\langle c \mid x_{0}\right\rangle_{a}$ and $\beta=$ $\left\langle a \mid x_{0}\right\rangle_{c}$. Then the continuity of the Gromov product (see Theorem 6.3) implies

$$
\begin{aligned}
& \lim _{n \rightarrow \infty} \alpha_{n}=\alpha ; \\
& \lim _{n \rightarrow \infty} \beta_{n}=\beta .
\end{aligned}
$$

Define $t_{n}$ to be the real number satisfying

$$
\begin{array}{ll}
\Theta_{\left[\alpha_{n}, \beta_{n}\right]}\left(t_{n}\right)=\min \left\{2, \hat{D}\left(a_{n}, c_{n}\right) / 2\right\}-\left\langle c_{n} \mid x_{0}\right\rangle_{a_{n}} & c_{n} \in X ; \\
\Theta_{\left[\alpha_{n}, \beta_{n}\right]}\left(t_{n}\right)=2-\left\langle c_{n} \mid x_{0}\right\rangle_{a_{n}} & c_{n} \in \partial X .
\end{array}
$$

Define $t$ to be the real number satisfying

$$
\begin{aligned}
& \Theta_{[\alpha, \beta]}(t)=\min \{2, \hat{D}(a, c) / 2\}-\left\langle c \mid x_{0}\right\rangle_{a} \quad c \in X ; \\
& \Theta_{[\alpha, \beta]}(t)=2-\left\langle c \mid x_{0}\right\rangle_{a} \quad c \in \partial X \text {. }
\end{aligned}
$$

Then $\iota_{x_{0}}\left(a_{n}, c_{n}\right)=\left(a_{n}, c_{n}, t_{n}\right)$ and $\iota_{x_{0}}(a, c)=(a, c, t)$. Because of Theorem 7.3 it suffices to show that $\lim _{n \rightarrow \infty} t_{n}=t$ holds. From Lemma 8.3 applied in the case $b=x_{0}$ we conclude $-\hat{D}\left(a_{n}, x_{0}\right) \leq t_{n} \leq 5 / 2$ and $-\widehat{D}\left(a, x_{0}\right) \leq t \leq 5 / 2$. Hence we can assume without loss of generality for all $n \geq 0$

$$
-\widehat{D}\left(a, x_{0}\right)-1 \leq t_{n}, t \leq 5 / 2 \text {. }
$$


We conclude from the Mean Value Theorem for some number $\rho \in[0,1]$

$$
\Theta_{[\alpha, \beta]}\left(t_{n}\right)-\Theta_{[\alpha, \beta]}(t)=\Theta_{[\alpha, \beta]}^{\prime}\left(\rho \cdot t_{n}+(1-\rho) t\right) \cdot\left(t_{n}-t\right) .
$$

Choose a constant $C>0$ such that $\Theta_{[\alpha, \beta]}^{\prime}(s) \geq C^{-1}$ holds for $s \in\left[-\widehat{D}\left(a, x_{0}\right)-1,5 / 2\right]$. Since $\rho \cdot t_{n}+(1-\rho) t$ lies in $\left[-\widehat{D}\left(a, x_{0}\right)-1,5 / 2\right]$, we conclude for all $n \geq 0$

$$
\left|t_{n}-t\right| \leq C \cdot\left|\Theta_{[\alpha, \beta]}\left(t_{n}\right)-\Theta_{[\alpha, \beta]}(t)\right| .
$$

We get from the triangle inequality

$$
\left|\Theta_{[\alpha, \beta]}\left(t_{n}\right)-\Theta_{[\alpha, \beta]}(t)\right| \leq\left|\Theta_{\left[\alpha_{n}, \beta_{n}\right]}\left(t_{n}\right)-\Theta_{[\alpha, \beta]}\left(t_{n}\right)\right|+\left|\Theta_{\left[\alpha_{n}, \beta_{n}\right]}\left(t_{n}\right)-\Theta_{[\alpha, \beta]}(t)\right|
$$

and hence

$$
\left|t_{n}-t\right| \leq C \cdot\left(\left|\Theta_{\left[\alpha_{n}, \beta_{n}\right]}\left(t_{n}\right)-\Theta_{[\alpha, \beta]}\left(t_{n}\right)\right|+\left|\Theta_{\left[\alpha_{n}, \beta_{n}\right]}\left(t_{n}\right)-\Theta_{[\alpha, \beta]}(t)\right|\right) .
$$

Since $a_{n}, a \in X$, we have $-\infty<\alpha_{n}$ and $-\infty<\alpha$. We have $\beta<\infty$ if and only if $c \in X$ and $\beta_{n}<\infty$ if and only if $c_{n} \in X$. If $\beta=\infty$, we can assume without loss of generality $5 / 2 \leq \beta_{n}$ and hence $t_{n} \leq \beta_{n}$ for all $n \geq 0$. We conclude

$$
\begin{array}{lll}
\left|\Theta_{\left[\alpha_{n}, \beta_{n}\right]}\left(t_{n}\right)-\Theta_{[\alpha, \beta]}\left(t_{n}\right)\right| \leq \max \left\{\left|\alpha_{n}-\alpha\right|,\left|\beta_{n}-\beta\right|\right\} & \beta_{n}, \beta<\infty ; \\
\left|\Theta_{\left[\alpha_{n}, \beta_{n}\right]}\left(t_{n}\right)-\Theta_{[\alpha, \beta]}\left(t_{n}\right)\right| \leq\left|\alpha_{n}-\alpha\right| & & \beta_{n}=\beta=\infty ; \\
\left|\Theta_{\left[\alpha_{n}, \beta_{n}\right]}\left(t_{n}\right)-\Theta_{[\alpha, \beta]}\left(t_{n}\right)\right| \leq\left|\alpha_{n}-\alpha\right|+e^{t_{n}-\beta_{n}} & & \beta_{n}<\infty, \beta=\infty .
\end{array}
$$

from Lemma 6.7 (vii) and (viii) and (the triangle inequality in the last case). Since $t_{n} \leq 5 / 2, \lim _{n \rightarrow \infty} \alpha_{n}=\alpha$ and $\lim _{n \rightarrow \infty} \beta_{n}=\beta$, we conclude

$$
\lim _{n \rightarrow \infty}\left|\Theta_{\left[\alpha_{n}, \beta_{n}\right]}\left(t_{n}\right)-\Theta_{[\alpha, \beta]}\left(t_{n}\right)\right|=0
$$

Since $\hat{D}$ and the Gromov product are continuous, we get using (8.6) and (8.7)

$$
\lim _{n \rightarrow \infty}\left|\Theta_{\left[\alpha_{n}, \beta_{n}\right]}\left(t_{n}\right)-\Theta_{[\alpha, \beta]}(t)\right|=0 .
$$

Now (8.8) implies $\lim _{n \rightarrow \infty} t_{n}=t$. This finishes the proof of Lemma 8.5.

Theorem 8.9 (Flow estimate for $\iota$ ) Let $\lambda \in\left(e^{-1}, 1\right)$ and $T \in[0, \infty)$ be the constants depending only on $X$ which appear in Proposition 6.4. Consider $a, b \in X$ and $c \in \bar{X}$. Put

$$
N=2+\frac{2}{\lambda^{T} \cdot(-\ln (\lambda))} .
$$

Then there exists a real number $\tau_{0}$ such that

$$
\left|\tau_{0}\right| \leq 2 \cdot \hat{D}(a, b)+5
$$


and for all $\tau \in \mathbb{R}$

$$
d_{F S, x_{0}}\left(\phi_{\tau \iota x_{0}}(a, c), \phi_{\tau+\tau_{0}} \iota_{x_{0}}(b, c)\right) \leq \frac{N}{1-\ln (\lambda)^{2}} \cdot \lambda^{-\widehat{D}(a, b)} \cdot \lambda^{\tau} .
$$

\section{Proof Put}

$$
\begin{aligned}
& t:= \begin{cases}\left(\Theta_{\left[-\left\langle c \mid x_{0}\right\rangle_{a},\left\langle a \mid x_{0}\right\rangle_{c}\right]}\right)^{-1}\left(\min \{2, \hat{D}(a, c) / 2\}-\left\langle c \mid x_{0}\right\rangle_{a}\right) & \text { if } c \in X \text { and } a \neq c ; \\
\left(\Theta_{\left[-\left\langle c \mid x_{0}\right\rangle_{a}, \infty\right]}\right)^{-1}\left(2-\left\langle c \mid x_{0}\right\rangle_{a}\right) & \text { if } c \in \partial X ; \\
\left\langle a, c \mid b, x_{0}\right\rangle=0 & \text { if } a=c ;\end{cases} \\
& s:= \begin{cases}\left(\Theta_{\left[-\left\langle c \mid x_{0}\right\rangle_{b},\left\langle b \mid x_{0}\right\rangle_{c}\right]}\right)^{-1}\left(\min \{2, \hat{D}(b, c) / 2\}-\left\langle c \mid x_{0}\right\rangle_{b}\right) & \text { if } c \in X \text { and } b \neq c ; \\
\left(\Theta_{\left[-\left\langle c \mid x_{0}\right\rangle_{b}, \infty\right]}\right)^{-1}\left(2-\left\langle c \mid x_{0}\right\rangle_{b}\right) & \text { if } c \in \partial X ; \\
\left\langle b, c \mid a, x_{0}\right\rangle=0 & \text { if } b=c .\end{cases}
\end{aligned}
$$

Put

$$
\tau_{0}=t-s-\left\langle a, b \mid c, x_{0}\right\rangle .
$$

We have by definition

$$
\begin{aligned}
\iota_{x_{0}}(a, c) & =(a, c, t) ; \\
\iota_{x_{0}}(b, c) & =(b, c, s) ; \\
\tau_{0} & =t-s-\left\langle a, b \mid c, x_{0}\right\rangle=\left(t-\left\langle a, c \mid b, x_{0}\right\rangle\right)-\left(s-\left\langle b, c \mid a, x_{0}\right\rangle\right) .
\end{aligned}
$$

We conclude from Theorem 7.1

$$
d_{F S, x_{0}}\left(\phi_{\tau} \iota x_{0}(a, c), \phi_{\tau+\tau_{0}} \iota_{x_{0}}(b, c)\right) \leq \frac{N}{1-\ln (\lambda)^{2}} \cdot \lambda^{\left(t-\left\langle a, c \mid b, x_{0}\right\rangle\right)} \cdot \lambda^{\tau} .
$$

We have

$$
d_{F S, x_{0}}((a, c, t),(a, a, 0))= \begin{cases}\min \{2, \widehat{D}(a, c) / 2\} & c \in X \\ 2 & c \in \partial X\end{cases}
$$

and

$$
d_{F S, x_{0}}((b, c, s),(b, b, 0))= \begin{cases}\min \{2, \hat{D}(b, c) / 2\} & c \in X ; \\ 2 & c \in \partial X .\end{cases}
$$

We conclude from Lemma 8.3 and the definition of $t$ and $s$ respectively that

$$
\begin{aligned}
& -\hat{D}(a, b) \leq t-\left\langle a, c \mid b, x_{0}\right\rangle \leq 5 / 2 \\
& -\hat{D}(a, b) \leq s-\left\langle b, c \mid a, x_{0}\right\rangle \leq 5 / 2
\end{aligned}
$$

holds. This finishes the proof of Theorem 8.9.

We can now prove the flow estimate from the introduction. 
Proof of Theorem 1.5 Define $j: G \times \bar{X} \rightarrow F S(X)$ by $j(g, c):=\iota_{x_{0}}\left(g x_{0}, c\right)$. It follows from Lemma 8.5 that $j$ is continuous and $G$-equivariant with respect to the diagonal $G$-action on the source. Let $C:=\max \left\{\widehat{D}\left(x_{0}, s x_{0}\right) \mid s \in S\right\}$ where $S$ is the generating set $S$ of $G$ used to define the word metric $d_{G}$ on $G$. Then

$$
\widehat{D}\left(g x_{0}, h x_{0}\right) \leq C d_{G}(g, h) \quad \forall g, h \in G .
$$

Let $\alpha>0$ be given. Let $\lambda \in\left(e^{-1}, 1\right)$ and $T \in[0, \infty)$ be the constants depending only on $X$ which appear in Proposition 6.4. Let $N$ be the number defined in Theorem 8.9. Define

$$
\begin{aligned}
\beta(\alpha) & :=2 C \alpha+5 \\
f_{\alpha}(\tau) & :=\frac{N}{1-\ln (\lambda)^{2}} \cdot \lambda^{-C \alpha} \cdot \lambda^{\tau} .
\end{aligned}
$$

It follows from Theorem 8.9 that $\beta(\alpha)$ and $f_{\alpha}$ satisfy the assertion of Theorem 1.5.

\section{Further properties of the flow space}

Theorem 9.1 Let $X$ be a hyperbolic complex with base point $x_{0} \in X$. Suppose that $G$ acts on $X$ by simplicial automorphisms such that every isotropy group is finite. Then:

(i) The metric space $\left(F S(X), d_{F S, x_{0}}(X)\right)$ is proper;

(ii) The induced $G$-action on the flow space $\left(F S(X), d_{F S, x_{0}}\right)$ is proper;

(iii) If $G$ acts cocompactly on $X$, then $G$ acts cocompactly on $F S(X)$.

A $G$-space $Y$ is called proper if for every $y \in Y$ there exists an open neighborhood $U$ such that the set $\{g \in G \mid g \cdot U \cap U \neq \varnothing\}$ is finite. Proper implies that all isotropy groups are finite. The converse is not true in general but is true if $Y$ is a $G-C W$-complex [13, Theorem 1.23 on page 18]. If $\left(Y, d_{Y}\right)$ is a metric space and $G$ acts by isometries, the $G$-action is proper if and only if for every $y \in Y$ there exists an $\epsilon$ such that the set $\left\{g \in G \mid g \cdot B_{\epsilon}(y) \cap B_{\epsilon}(y) \neq \varnothing\right\}$ is finite, where $B_{\epsilon}(y)=\left\{z \in Y \mid d_{Y}(y, z)<\epsilon\right\}$. A metric space $\left(Y, d_{Y}\right)$ is called proper if and only if $\overline{B_{\epsilon}(y)}=\left\{z \in Y \mid d_{Y}(y, z) \leq \epsilon\right\}$ is compact for all $y \in Y$ and $\epsilon \geq 0$.

The elementary proof of the next result is left to the reader.

Lemma 9.2 Let $\left(Y, d_{Y}\right)$ be a proper metric space. Let $G$ act on $Y$ by isometries. Then the $G$-action on $Y$ is proper if and only if for every $C>0$ and $y \in Y$ the set $\left\{g \mid d_{Y}(g \cdot y, y) \leq C\right\}$ is finite. 
Now we are ready to prove Theorem 9.1.

Proof (i) This is proven in [14, Proposition 54, page 464].

(ii) Mineyev [14, page 457] constructs a map

$$
\Psi: F S(X) \rightarrow X
$$

such that there exists constants $K_{1}$ and $K_{2}$ depending only on $X$ such that for all $v, w \in F S(X)$ and $g \in G$ we have

$$
\begin{array}{r}
\left|d_{F S, x_{0}}(v, w)-\hat{D}(\Psi(v), \Psi(w))\right| \leq K_{1} ; \\
\hat{D}(\Psi(g \cdot v), g \cdot \Psi(v)) \leq K_{2} .
\end{array}
$$

Compare also [14, Proposition 43 on page 458].

We conclude for $v \in F S(X)$ and $g \in G$

$$
\begin{aligned}
& \widehat{D}(g \cdot \Psi(v), \Psi(v)) \\
& \leq \widehat{D}(\Psi(g \cdot v), \Psi(v))+\widehat{D}(\Psi(g \cdot v), g \cdot \Psi(v)) \\
& =d_{F S, x_{0}}(g \cdot v, v)-d_{F S, x_{0}}(g \cdot v, v)+\widehat{D}(\Psi(g \cdot v), \Psi(v))+\widehat{D}(\Psi(g \cdot v), g \cdot \Psi(v)) \\
& \leq d_{F S, x_{0}}(g \cdot v, v)+\left|d_{F S, x_{0}}(g \cdot v, v)-\hat{D}(\Psi(g \cdot v), \Psi(v))\right|+\widehat{D}(\Psi(g \cdot v), g \cdot \Psi(v)) \\
& \leq d_{F S, x_{0}}(g \cdot v, v)+K_{1}+K_{2} .
\end{aligned}
$$

There exist real numbers $A \geq 1$ and $B \geq 0$ depending only on $X$ such that for all $x_{1}, x_{2} \in X$ we have

$$
A^{-1} \widehat{D}\left(x_{1}, x_{2}\right)-B \leq d\left(x_{1}, x_{2}\right) \leq A \cdot \widehat{D}\left(x_{1}, x_{2}\right)+B
$$

where $d$ is the word metric; compare the beginning of Section 6.1. Hence we get

$$
\begin{aligned}
d(g \cdot \Psi(v), \Psi(v)) & \leq A \cdot \widehat{D}(g \cdot \Psi(v), \Psi(v))+B \\
& \leq A \cdot\left(d_{F S, x_{0}}(g \cdot v, v)+K_{1}+K_{2}\right)+B .
\end{aligned}
$$

Consider $v \in F S(X)$ and $C \geq 0$. Since $G$ acts properly on $X$ and $(X, d)$ is a proper metric space, Lemma 9.2 implies that the set

$$
\left\{g \in G \mid d(g \cdot \Psi(v), \Psi(v)) \leq A \cdot\left(C+K_{1}+K_{2}\right)+B\right\}
$$

is finite. Since this set contains $\left\{g \in G \mid d_{F S, x_{0}}(g \cdot v, v) \leq C\right\}$, also the latter set is finite. Hence the $G$-action on $\left(F S(X), d_{F S, x_{0}}\right)$ is proper by Lemma 9.2.

(iii) Since $G$ acts simplicially and cocompactly on $X$, we can find a compact subset $C \subseteq X$ such that $G \cdot C=X$. Consider $D=\left\{x \in F S(X) \mid \widehat{D}(\Psi(x), C) \leq K_{2}\right\}$. Since 
$C$ is compact, its diameter $\operatorname{diam}(C)$ is finite. Since for $y, z \in D$ we get

$$
d_{F S, x_{0}}(y, z) \leq \widehat{D}(\Psi(y), \Psi(z))+K_{1} \leq \operatorname{diam}(C)+2 K_{2}+K_{1}
$$

the set $D$ has finite diameter. Since $F S(X)$ is proper as a metric space by assertion (i), the closure of $D$ is a compact subset of $F S(X)$. Next we show $G \cdot D=F S(X)$. Consider $x \in F S(X)$. Choose $g \in G$ such that $g^{-1} \Psi(x) \in C$. From $\widehat{D}\left(\Psi\left(g^{-1} x\right), g^{-1} \Psi(x)\right) \leq$ $K_{2}$ we conclude $g^{-1} x \in D$. This implies $x \in g \cdot D \subseteq G \cdot D$. Hence $\bar{D}$ is a compact subset of $F S(X)$ with $G \cdot \bar{D}=F S$. Therefore $G$ acts on $F S$ cocompactly. This finishes the proof of Theorem 9.1.

The following facts are well-known. We include a proof for the convenience of the reader.

Lemma 9.3 Let $X$ be a $\delta$-hyperbolic complex in the sense of Section 6.1. Let $\bar{X}$ be the compactification of $X$ in the sense of Gromov. Then

(i) $\bar{X}$ is locally connected;

(ii) $\bar{X}$ has finite covering dimension.

Proof We start by reviewing the topology of $\bar{X}$ following [5, p 429]. Recall that $X^{(1)}$ denotes the 1-skeleton of $X$. A generalized ray $c: I \rightarrow X^{(1)}$ is a geodesic with respect to the word metric $d_{\text {word }}$, where $I=[0, R]$ for $R \geq 0$ or $I=[0, \infty)$. In the later case $c$ will be called a geodesic ray. If $I=[0, R]$ it is convenient to write $c(t)=c(R)$ for $t \geq r$. Two geodesic rays $c, c^{\prime}$ are called equivalent if there is $C>0$ such that $d_{\text {word }}\left(c(t), c^{\prime}(t)\right)<C$ for all $t \in[0, \infty) . \partial X=\partial X^{(1)}$ is the set of all equivalence classes of such geodesic rays. For a geodesic ray $c$ we denote by $c(\infty)$ the point in $\partial X$ determined by $c$. Fix a base point $x_{0} \in X^{(0)}$ and $k>2 \delta$. Every point in $\partial X$ can be written as $c(\infty)$ where $c$ is a geodesic ray starting at $x_{0}$ [5, Lemma 3.1 on page 427]. For a geodesic ray $c$ starting at $x_{0}$ and $n \in \mathbb{N}$ let $V_{n}^{(1)}(c)$ denote the set of all $c^{\prime}(\infty)$ where $c^{\prime}$ is a generalized ray starting at $x_{0}$ with $d_{\text {word }}\left(c(n), c^{\prime}(n)\right)<k$. (Such a generalized ray may end in $X$.) Let $V_{n}(c)$ be the union of $V_{n}^{(1)}(c)$ with the smallest subcomplex of $X$ containing $V_{n}^{(1)}(c) \cap X$. The topology on $\bar{X}=X \cup \partial X$ is now defined as follows: a $U \subset \bar{X}$ is open if and only if the following two conditions hold:

- $U \cap X$ is open in $X$.

- If $c$ is a geodesic ray starting at $x_{0}$ and $c(\infty) \in U$ then there is $n \in \mathbb{N}$ such that $V_{n}(c) \subset U$. 
We now prove (i). Obviously $X$ is locally connected as it is a $C W$-complex. It suffices to show for every $x \in \partial X=\bar{X}-X$ that there is a (not necessarily open) connected neighborhood (see [15, Exercise 10 on page 163]). But this follows because the $V_{n}(c)$ from above are connected.

Next we prove (ii). Let $D \in \mathbb{N}$ be the maximal number of points in $X^{(0)}$ that are contained in a ball of radius $k+2 \delta$. This is a finite number because $X$ is uniformly locally finite. Because $X$ has finite covering dimension and $\partial X$ is compact, it suffices to show the following: For every finite collection $\mathcal{U}$ of open subsets of $\bar{X}$ that covers $\partial X$ there is an $(D-1)$-dimensional refinement of $\mathcal{U}$ that still covers $\partial X$.

We will need the following observation: Let $c, c^{\prime}$ be two geodesics starting at $x_{0}$. Let $x$ be the endpoint of $c$ and $x^{\prime}$ be the endpoint of $c^{\prime}$. If $N$ is such that $d(c(N), x)>$ $d\left(x, x^{\prime}\right)+\delta$, then $d\left(c(N), c^{\prime}(N)\right) \leq 2 \delta$. This is an easy application of the condition that all geodesic triangles in $X^{(1)}$ are $\delta$-thin.

For $N \in \mathbb{N}$ consider the set $S_{N}$ of all $c(N)$ where $c$ is a geodesic ray in $X^{(1)}$ starting at $x_{0}$. This is a finite subset of $X^{(0)}$ because $X$ is locally finite. For every $x \in S_{N}$ pick a geodesic ray $c_{x}$ starting at $x_{0}$ such that $c_{x}(N)=x$. Denote by $B$ the close ball of radius $n+\delta$ around $x_{0}$. Let $U_{x}:=\left(V_{N}\left(c_{x}\right)\right)^{\circ}-B$. Then the collection $\mathcal{U}_{N}:=\left\{U_{x} \mid x \in S_{N}\right\}$ covers $\partial X$. Let $z \in \bar{X}-B$ and choose $x \in S_{N}$ such that $x$ lies on a geodesic from $x_{0}$ to $z$. Using the above observation it is not hard to show that for every $x^{\prime} \in S_{N}$ with $z \in U_{x^{\prime}}$ we have $d\left(x, x^{\prime}\right)<k+2 \delta$. Therefore the dimension of $\mathcal{U}_{N}$ is bounded by $D-1$.

It remains to show that for sufficiently large $N$, the collection $\mathcal{U}_{N}$ will be a refinement of the given collection $\mathcal{U}$. Let $R>k+3 \delta, R \in \mathbb{N}$. By the definition of the topology of $\partial X$ and because $\partial X$ is compact, there are $M_{1}, \ldots, M_{n} \in \mathbb{N}$ and geodesic rays $c_{1}, \ldots, c_{n}$ starting at $x_{0}$ such that

- $\partial X \subset V_{M_{1}+R}\left(c_{1}\right) \cup \cdots \cup V_{M_{n}+R}\left(c_{n}\right)$;

- for every $i=1, \ldots, n$ there is $U \in \mathcal{U}$ such that $V_{M_{i}}\left(c_{i}\right) \subset U$.

Let $M:=\max \left\{M_{1}, \ldots, M_{n}\right\}$. Two applications of the above observation give the following: If $c$ is a geodesic ray starting at $x_{0}$ such that $c(\infty) \in V_{M_{i}+R}\left(c_{i}\right)$, then $V_{M+2 R}(c) \subset V_{M_{i}}\left(c_{i}\right)$. Thus $\mathcal{U}_{M+2 R}$ is a refinement of $\mathcal{U}$.

We can now check the additional properties stated in Section 1.2.

Proof of Proposition 1.6 (i) This follows from [5, Theorem 3.2 on page 459]. 
(ii) By Lemma 9.3 $\bar{X}$ has finite covering dimension and is locally connected. It follows therefore from Theorem 7.3 that $F S(X)-F S(X)^{\mathbb{R}}=F S(X)-X$ is locally connected and has finite covering dimension.

(iii) This follows from Theorem 9.1.

(iv) For $g \in G$ its translation length on $\left(F S(X), d_{F S(X), x_{0}}\right)$ is defined as

$$
l(g):=\lim _{n \rightarrow \infty} d_{F S, x_{0}}\left(g^{n}(a, b, t),(a, b, t)\right) / n .
$$

By the triangle inequality this definition does not depend on the choice of $(a, b, t) \in$ $F S(X)$. In particular, it depends only on the conjugacy class of $g$. Since the isometric $G$-action on $F S(X)$ is cocompact and proper, $\left(G, d_{G}\right)$ is quasi-isometric to $\left(F S, d_{F S, x_{0}}\right)$. Thus, there are constants $A \geq 1, B>0$ such that

$$
A \cdot l(g)+B \geq \tau(g):=\lim _{n \rightarrow \infty} d_{G}\left(g^{n}, 1_{G}\right) .
$$

( $\tau(g)$ is the translation length of $g$ on $\left.\left(G, d_{G}\right).\right)$ By [5, Proposition 3.15 on page 465] for fixed $C>0$ the number of conjugacy classes whose translation length on $\left(G, d_{G}\right)$ is no more than $C$ is finite. We conclude that the same holds for the translation length on $\left(F S(X), d_{F S, x_{0}}\right)$.

Fix $C>0$. Let $\mathcal{L}$ be the set of all orbits $L$ of the flow $\phi_{\tau}$ on $F S(X)$ with $0<$ $\operatorname{per}_{\phi}^{G}(L) \leq C$, see Definition 2.15. Every $L \in \mathcal{L}$ is a line $\left(a_{L}, b_{L}\right)_{F S(X)}$ with $a_{L}$, $b_{L} \in \bar{X}$. For $L \in \mathcal{L}$ there is $g_{L} \in G$ such that

$$
g_{L} \cdot\left(a_{L}, b_{L}, t\right)=\left(a_{L}, b_{L}, t+\operatorname{per}_{\phi}^{G}(L)\right)
$$

for $\left(a_{L}, b_{L}, t\right) \in L$. In particular $g_{L} \cdot a_{L}=a_{L}, g_{L} \cdot b_{L}=b_{L}$. If $a_{L} \in X$ or $b_{L} \in X$, then $g_{L}$ has finite order because the action of $G$ on $X$ is proper. But this would imply $\operatorname{per}_{\phi}^{G}(L)=0$. Therefore $a_{L}, b_{L} \in \partial X$. By Lemma $6.16 l\left(g_{L}\right)=\operatorname{per}_{\phi}^{G}(L) \leq C$. Recall that $\partial X \cong \partial G$, because $X$ is quasi-isometric to $G$ [5, Theorem 3.9 on page 430]. Because every $g \in G$ has at most 2 fixed points on $\partial G$ [9, 20.- Corollaire on page 149] the map $L \mapsto g_{L}$ is injective. Because there are only finitely many conjugacy classes of translation length $\leq C$ this means that $G \backslash \mathcal{L}$ is finite. This is what we needed to prove.

\section{References}

[1] A Bartels, Squeezing and higher algebraic K-theory, K-Theory 28 (2003) 19-37 MR1988817

[2] A Bartels, T Farrell, L Jones, H Reich, On the isomorphism conjecture in algebraic K-theory, Topology 43 (2004) 157-213 MR2030590 
[3] A Bartels, W Lück, H Reich, The K-theoretic Farrell-Jones conjecture for hyperbolic groups, to appear in Invent. Math.

[4] A Bartels, D Rosenthal, On the $K$-theory of groups with finite asymptotic dimension, J. Reine Angew. Math. 612 (2007) 35-57 MR2364073

[5] M R Bridson, A Haefliger, Metric spaces of non-positive curvature, Grund. der Math. Wissenschaften [Fund. Principles of Math. Sciences] 319, Springer, Berlin (1999) MR1744486

[6] G Carlsson, B Goldfarb, The integral $K$-theoretic Novikov conjecture for groups with finite asymptotic dimension, Invent. Math. 157 (2004) 405-418 MR2076928

[7] F T Farrell, L E Jones, K-theory and dynamics. I, Ann. of Math. (2) 124 (1986) 531-569 MR866708

[8] F T Farrell, LE Jones, Isomorphism conjectures in algebraic $K$-theory, J. Amer. Math. Soc. 6 (1993) 249-297 MR1179537

[9] E Ghys, P de la Harpe, editor, Sur les groupes hyperboliques d'après Mikhael Gromov, Progress in Mathematics 83, Birkhäuser, Boston (1990) MR1086648Papers from the Swiss Seminar on Hyperbolic Groups held in Bern, 1988,

[10] M Gromov, Hyperbolic groups, from: "Essays in group theory”, Math. Sci. Res. Inst. Publ. 8, Springer, New York (1987) 75-263 MR919829

[11] M Gromov, Asymptotic invariants of infinite groups, from: "Geometric group theory, Vol. 2 (Sussex, 1991)", London Math. Soc. Lecture Note Ser. 182, Cambridge Univ. Press (1993) 1-295 MR1253544

[12] M Gromov, Metric structures for Riemannian and non-Riemannian spaces, english edition, Modern Birkhäuser Classics, Birkhäuser, Boston (2007) MR2307192Based on the 1981 French original, With appendices by M Katz, P Pansu and S Semmes, Translated from the French by S M Bates

[13] W Lück, Transformation groups and algebraic $K$-theory, Lecture Notes in Math. 1408, Springer, Berlin (1989) MR1027600Mathematica Gottingensis

[14] I Mineyev, Flows and joins of metric spaces, Geom. Topol. 9 (2005) 403-482 MR2140987

[15] J R Munkres, Topology: a first course, Prentice-Hall, Englewood Cliffs, N.J. (1975) MR0464128

[16] R S Palais, On the existence of slices for actions of non-compact Lie groups, Ann. of Math. (2) 73 (1961) 295-323 MR0126506

[17] J Roe, Hyperbolic groups have finite asymptotic dimension, Proc. Amer. Math. Soc. 133 (2005) 2489-2490 MR2146189

[18] R Sauer, Amenable covers, volume and $L^{2}$-Betti numbers of aspherical manifolds arXiv: math.AT/0605627 
[19] G Yu, The Novikov conjecture for groups with finite asymptotic dimension, Ann. of Math. (2) 147 (1998) 325-355 MR1626745

AB, WL: Westfälische Wilhelms-Universität Münster, Mathematisches Institut Einsteinstr. 62, D-48149 Münster, Germany

HR: Heinrich-Heine-Universität Düsseldorf, Mathematisches Institut Universitätsstr. 1, D-40225 Düsseldorf, Germany

a.bartels@uni-muenster.de, lueck@math.uni-muenster.de, reich@math.uni-duesseldorf.de

http://www.math.uni-muenster.de/u/bartelsa/bartels, http://

www.math.uni-muenster.de/u/lueck, http://reh.math.uni-duesseldorf.de/ $\% 7$ Ereich/

Proposed: Martin Bridson

Seconded: Steve Ferry, Ralph Cohen
Received: 28 September 2006

Accepted: 7 February 2008 